\title{
Concurrent eruptions at Etna, Stromboli, and Vulcano: casualty or causality?
}

\author{
Andrea Billi and Renato Funiciello \\ Dipartimento di Scienze Geologiche, Università «Roma Tre», Roma, Italy
}

\begin{abstract}
Anecdotes of concurrent eruptions at Etna, Stromboli, and Vulcano (Southern Italy) have persisted for more than 2000 years and volcanologists in recent and past times have hypothesized a causal link among these volcanoes. Here this hypothesis is tested. To introduce the problem and provide examples of the type of expected volcanic phenomena, narratives of the most notable examples of concurrent eruptions are provided. Then the frequency of eruptions at each individual volcano is analysed for about the last 300 years and the expected probability of concurrent eruptions is calculated to compare it to the observed probability. Results show that the occurrence of concurrent eruptions is often more frequent than a random probability, particularly for the Stromboli-Vulcano pair. These results are integrated with a statistical analysis of the earthquake catalogue to find evidence of linked seismicity in the Etnean and Aeolian areas. Results suggest a moderate incidence of non-random concurrent eruptions, but available data are temporally limited and do not allow an unequivocal identification of plausible triggers; our results, however, are the first attempt to quantify a more-than-2000-years-old curious observation and constitute a starting point for more sophisticated analyses of new data in the future. We look forward to our prediction of a moderate incidence of concurrent eruptions being confirmed or refuted with the passage of time and occurrence of new events.
\end{abstract}

Key words Etna - Stromboli - Vulcano - eruption, and earthquake

\section{Introduction}

Earthquake-eruption and eruption-eruption triggering mechanisms are the subject of intense debate and represent the new frontier for several geophysicists and volcanologists (Kanamori and Givens, 1982; Kimura, 1996; Brodsky et al., 1998, 2000; Gudmundsson and Brenner, 2003; Marzocchi et al., 2004; Gudmundsson, 2006; Feuillet et al., 2006; Madonia

Mailing address: Andrea Billi, Dipartimento di Scienze Geologiche, Università «Roma Tre», Largo S. L. Murialdo 1, 00146 Roma, Italy; tel: +39 0657338016; fax: +39 0657338201; e-mail: billi@uniroma3.it et al., 2008). In recent years, a few case histories of eruptions that triggered earthquakes (Jacques et al., 1996; Azzaro et al., 2001; Walter and Amelung, 2006) or other eruptions (Miklius and Cervelli, 2003; Gudmundsson and Andrew, 2007), and of eruptions that hindered earthquakes (Walter and Amelung, 2004) have been reported. In contrast, several case histories of large earthquakes that triggered eruptions at near or remote volcanoes are now well documented (Carr, 1977; Marzocchi et al., 1993, 2002, 2004; Barrientos, 1994; Linde and Sacks, 1998; Nostro et al., 1998; Manga and Brodsky, 2006; Cigolini et al., 2007; Harris and Ripepe, 2007; Lemarchand and Grasso, 2007; Manga, 2007; Mellors et al., 2007; Walter and Amelung, 2007; Walter et al., 2007).

Eruptions triggering other eruptions at the Southern Italian volcanoes (i.e., Etna and Aeolian volcanoes, fig. 1) have been speculated since at least the $1^{\text {st }}$ Century BC (Diodorus 
Siculus in Bibliotheca Historica). Julius Solinus (in Collectanea Rerum Memorabilium, $4^{\text {th }}$ Century AD) wrote that there should have been subterranean conduits that linked and fed these volcanoes. de Dolomieu (1783) also hypothesized direct feeding relationships between Etna and the Aeolian volcanoes, whereas Mercalli (1879, 1888) supposed an indirect physical (i.e., dynamic) link between these volcanoes. Recently, Cigolini et al. (2007) provided evidence of linked volcanic phenomena at Etna and Aeolian volcanoes during 2002-2003.

Although Southern Italy is seismically active and several $M \geq 7$ earthquakes were recorded in historical times (Boschi et al., 1997; Neri et al., 2006; Basili et al., 2008), eruptions at Etna, Stromboli, and Vulcano triggered by large nearby earthquakes are not known. In particular, the six largest historical earthquakes (with epicentral MCS intensity $\geq$ IX) of eastern Sicily and southern Calabria (fig. 1) were not shortly followed by significant eruptions from the nearby volcanoes (table I), which, therefore, provide valuable case histories to study eruption triggers alternative to large nearby earthquakes (Sharp et al., 1981).

This paper searches for evidence for a link between eruptions at Etna, Stromboli, and Vulcano. Our hypothesis is that concurrent activity at these volcanoes may occur at a frequency higher than expected based on the background rate. This hypothesis is explored in three different ways. The eruption catalogue is statistically analysed. The statistical method has the advantage of being quantitative, but the disadvantage of providing little insight into the plethora of behaviour that can fall under the heading of eruption. To fill out this story and provide examples of the type of phenomena expected, narratives of the most notable examples of concurrent activity are provided. Finally, the catalogue of instrumentally-recorded earthquakes is analysed to find evidence of linked seismicity in the Etnean and Aeolian areas. This paper extends previous studies on analogous subjects (e.g., Mulargia et al. 1985; Nercessian et al., 1991; Cardaci et al., 1993). Moreover, the problem of possible links between concurrent eruptions at nearby volcanoes has been previously studied in other regions (e.g., Klein, 1982; Bebbington and Lai, 1996).

\section{Geological setting}

Etna and Aeolian volcanoes (fig. 1) are located along the active convergent margin between the African and Eurasian plates in the central Mediterranean region (Malinverno and Ryan, 1986). A narrow remnant of the Ionian

Table I. Largest historical earthquakes of Sicily and southern Calabria (southern Italy, fig. 1) and subsequent eruptions at Etna, Stromboli, and Vulcano. Data are from Simkin and Siebert (1994) and from Boschi et al. (1997).

\begin{tabular}{lcll}
\hline \hline earthquake date & epicentral MCS intensity & epicentral area & subsequent eruptions \\
\hline 4 Feb. 1169 & XI & $\begin{array}{l}\text { Estern Sicily and } \\
\text { western Calabria }\end{array}$ & $\begin{array}{l}\text { a doubtful eruption } \\
\text { from Etna on Feb. 1169 }\end{array}$ \\
10 Dec. 1542 & IX-X & Southeastern Sicily & none \\
11 Jan. 1693 & XI & Southeastern Sicily & none \\
5 Mar. 1823 & X & Northeastern Sicily & none \\
28 Dec. 1908 & XI & Messina Straits & none \\
15 Oct. 1911 & X & Southeastern flank & \\
& & of Mt Etna & none \\
\hline
\end{tabular}




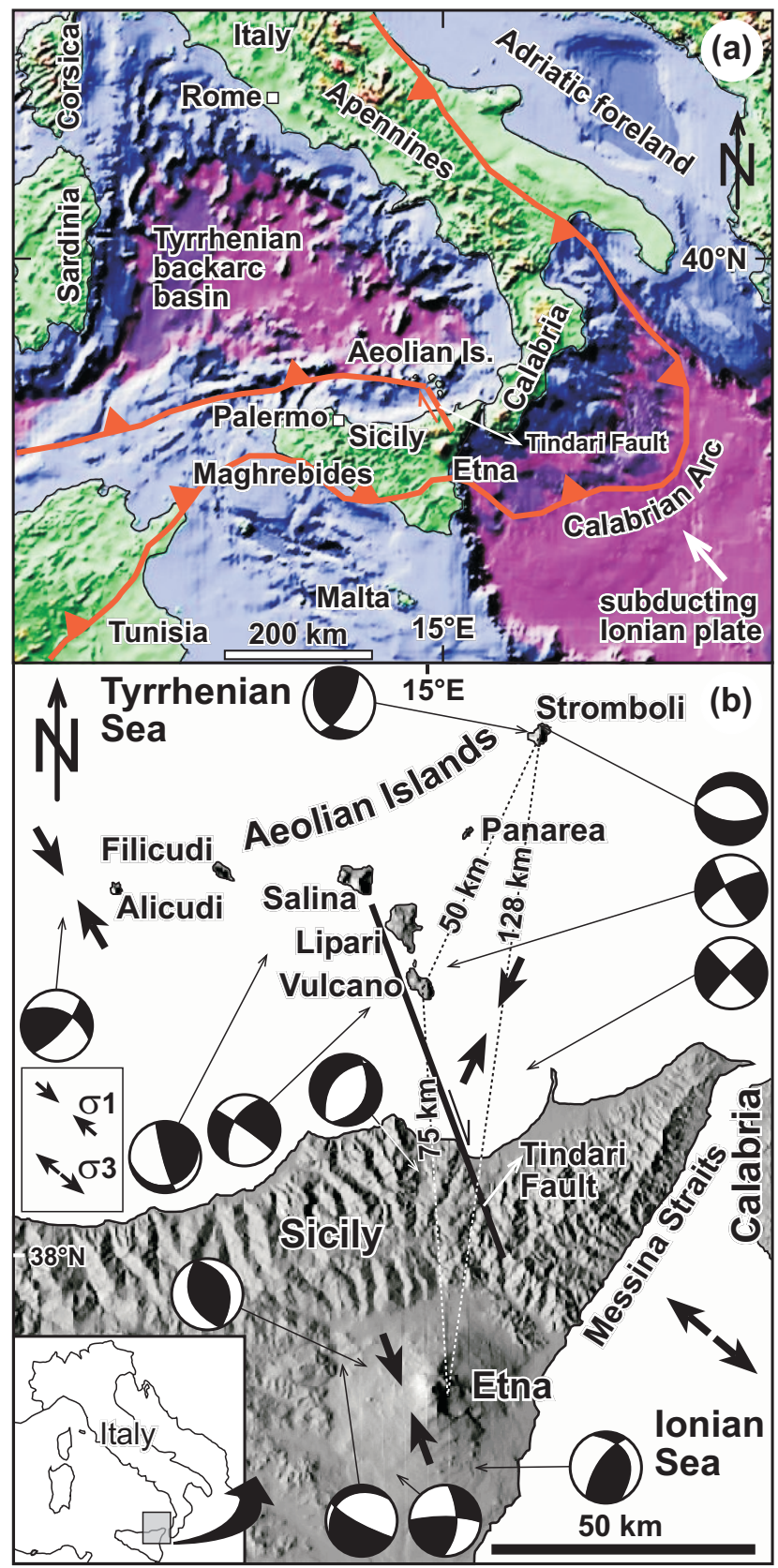

Fig. 1a,b. (a) Tectonic map of Southern Italy. Main fold-thrust belts occurring along the African-European plate boundary (i.e., Apennines, Calabrian Arc, and Maghrebides) are drawn in red. (b) Hillshade of north-eastern Sicily, including the studied volcanoes (i.e., Etna, Stromboli, and Vulcano). Note that Stromboli and Vulcano are included in the Aeolian Archipelago. Earthquake focal mechanisms $(M \geq 3.5)$ and arrows indicating active stresses are from Neri et al. (2005). 
slab (African affinity) is subducting toward the northwest beneath the Calabrian Arc, whereas large slab windows occur beneath Sicily and the southern Apennines, where subduction is mostly inactive (Gvirtzman and Nur, 2001; Faccenna et al., 2004, 2005). The Apennine and Maghrebian thrust-fold belt have grown since late Paleogene time along the Africa-Eurasia suture zone, with eastward and southward vergence, respectively. To the north of Sicily, the oceanic Tyrrhenian backarc basin started to form since about late Neogene time (Malinverno and Ryan, 1986; Dewey et al., 1989; Patacca et al., 1992).

Seismic and geodetic evidence from Sicily and surrounding regions shows that, in the central Mediterranean region, Africa and Eurasia are converging at a considerable rate (circa 3 $\mathrm{mm} / \mathrm{yr}$ ) and that both the Etnean and Aeolian areas are undergoing $\mathrm{N}$-S-oriented tectonic compression (fig. 1b; Barberi et al., 2000; Neri et al., 2003, 2005; D'Agostino and Selvaggi, 2004; Goes et al., 2004; Montone et al., 2004; Pondrelli et al., 2004). In the study area, main active tectonic elements are the roughly E-Wtrending thrust belt in the southern Tyrrhenian area and the NNW-striking Tindari Fault between the Aeolian Islands and Mount Etna (fig. 1). The compressional belt in the southern Tyrrhenian area accommodates most of the Africa-Eurasia ongoing convergence in the central Mediterranean area and is characterized by compressional and transpressional earthquakes with a maximum recorded magnitude of 5.9 (Goes et al., 2004; Billi et al., 2007). This belt involves the western islands of the Aeolian Archipelago, i.e., Alicudi and Filicudi, which are the summit of submarine volcanoes inactive since about mid-Pleistocene time. The Tindari Fault is the eastern boundary of the southTyrrhenian compressional belt and is characterized by right-lateral strike-slip earthquakes with a maximum recorded magnitude of 5.5. This fault involves the central islands of the Aeolian Archipelago (Salina, Lipari, and Vulcano) and extends toward the south near Mount Etna (Ghisetti, 1979; Tortorici et al., 1995; Ventura et al., 1999; Billi et al., 2006).

The Aeolian volcanoes (Alicudi, Filicudi, Lipari, Panarea, Salina, Stromboli, and Vulcano, fig. 1) form a high-K calc-alkaline island arc, whose activity started about $1.3 \mathrm{Myr}$ ago and is still active on at least three of the seven major islands, namely Lipari (last eruption in 729), Vulcano (last eruptions in 1888-1892), and Stromboli (Simkin and Siebert, 1994; Behncke, 2001). Hydrothermal activity offshore Panarea has occurred in historical times and in recent years (e.g., Gabbianelli et al., 1993; Esposito et al., 2006). Stromboli is characterized by a persistent volcanic activity consisting in continuous small explosions occurring at approximately regular intervals of a few minutes (i.e., strombolian activity). However, individual events, such as lava effusions and paroxysmal eruptions, occurred at Stromboli during recent and historical times (Rittmann, 1931; Simkin and Siebert, 1994). The volcanic activity of Vulcano has been characterized by powerful and impressive explosions (i.e., vulcanian eruptions) alternated with less frequent effusive events (Frazzetta et al., 1984; Simkin and Siebert, 1994).

Volcanism in the Etnean area started about $0.5 \mathrm{Myr}$ ago with tholeiitic magmas (Branca et al., 2008). The modern volcano formed by a succession of volcanic edifices consisting in alternating pyroclastic and effusive rocks (Gillot et al., 1994; Behncke, 2001; Tanguy et al., 2007; Branca et al., 2008). The volcanic activity of Etna is, in fact, mostly effusive; however, explosive activity has often occurred at the summit craters (e.g., Simkin and Siebert, 1994). Lateral effusive and mildly explosive eruptions have also been frequent at Mount Etna (Rittmann, 1964; Lanzafame et al., 2003; Alparone et al., 2005). The source of Etna magmatism is possibly connected with a mantle plume that Montelli et al. (2004) imaged above $1000 \mathrm{~km}$ using seismic tomography. Alternatively or complementarily, the voluminous melting under Mount Etna may result from suction of asthenospheric material induced by the backward migration of the descending Ionian slab (Gvirtzman and Nur, 1999).

\section{Examples of concurrent eruptions}

Eruption catalogues are notoriously subjective. Eruptive activity is gradational and activity during a given month may mean a variety of 
different phenomena. To fill out this story, before the statistical analysis of the eruption catalogue, brief narratives of four of the most notable examples of concurrent activity are provided.

Two of these episodes fall outside our statistical study period (see the following section) and three involve observations from another Aeolian volcano (i.e., Panarea), but these stories are anyhow included to provide examples of the type of phenomena expected.

The acronym VEI is henceforth used instead of volcanic explosivity index (Newhall and Self, 1982) and the related values are from Simkin and Siebert (1994).

\subsection{Concurrent activity during 2002-2003}

The 2002 eruption of Etna started the night between 26 and 27 October 2002 from the summit craters, concurrently with the formation of ground-surface fractures in this area. From the summit craters, fractures propagated toward the south and toward the northeast.

Newly generated vents and cones developed along these fractures.

For about two months since the onset of the eruption, voluminous emissions of ash occurred. Volcanic ashes fell in the nearby regions of Sicily and Calabria. After about 95 days of almost continuous eruption, on 29 January 2003, Etna ceased most of its effusive and explosive activity.

The 2002-2003 eruption of Etna was highly explosive, possibly one of the most explosive of the last 350 years.

Early in the morning of 3 November 2002, local fishermen observed spots of anomalous seawater boiling and high mortality of fishes offshore Panarea, and perceived smell of sulphurs. In particular, five major sites of gas emissions were found around the islets of Lisca Bianca, Bottaro, and Lisca Nera. At these sites, ascent of gas bubbles with diameter up to $1 \mathrm{~m}$ on the sea surface signalled the presence of submarine gas-emissions. With the exception of one site close to Lisca Bianca, these sites were previously unknown as gas-emitting spots. Gas emissions originated from NW- and NE-strik- ing fractures in volcanic rocks lying at depths between 8 and $30 \mathrm{~m}$ from the sea surface. Gas emissions offshore Panarea gradually ceased during the first months of 2003.

On 28 December 2002, after 17 years of mild although continuous volcanic activity, an intense eruption began at Stromboli. The eruption was preceded by seismic swarms and by an increase in volcanic tremors since 3 November 2002 , concurrently with the onset of gas emissions offshore Panarea.

Since 28 December 2002, lava flowed from the Stromboli summit crater into the sea along the steep northwest flank. Renewed lava flow occurred since 30 December 2002. The eruption mostly ceased by 5 April 2003, when a powerful explosion occurred at the summit crater of Stromboli.

The above information is mostly synthesized after Acocella et al. (2003); Dellino and Kyriakopoulos (2003); Patanè et al. (2003); Caliro et al. (2004); Calvari et al. (2005); Ripepe et al. (2005); Walter et al. (2005); Esposito et al. (2006), and Cigolini et al. (2007).

\subsection{Concurrent activity during 1886-1890}

From 10 January to 31 March 1886, strombolian activity occurred at Vulcano. On 22 January 1886, volcanic explosions occurred at Stromboli. On 18 May 1886, an eruption began at the summit craters of Mount Etna. This eruption was highly explosive (VEI $=3$ ).

During January, March, and November 1887 , volcanic explosions occurred at Stromboli. On 3 August 1888, an eruption began at Vulcano.

The eruptive activity of Vulcano ceased in March 1990. The 1888-1890 eruption of Vulcano was highly explosive $(\mathrm{VEI}=3)$. On 23 October 1888, volcanic explosions and lava fountains occurred at Stromboli. On 29 November 1888, offshore Vulcano, local fishermen observed boiling seawater and associated fish mortality, and perceived smell of sulphurs.

The above information are mostly synthesized after Mercalli (1888); Silvestri (1890, 1893); Baratta (1901) and Simkin and Siebert (1994). 


\subsection{Concurrent activity during 1865}

On 30 January 1865, an explosive (VEI $\geq 2$ ) eruption occurred at Etna. At the onset of the eruption, NE-striking fractures propagated across the summit crater of Mount Etna. Several vents and cones formed along these fractures. The effusive and explosive activity at Mount Etna lasted for about 90 days. Voluminous emissions of ash, which fell for about two months in the nearby regions of Sicily and Calabria, accompanied the eruption from the beginning. During the 1865 eruption of Etna, emissions of sulphurous gases occurred offshore Panarea. From 26 January to 2 February 1865, volcanic explosions occurred at Stromboli.

The above information is mostly synthesized after Fouqué (1865); Silvestri (1867); Mercalli (1879, 1888); Baratta (1901), and Simkin and Siebert (1994). Most volcanic phenomena occurred at Etna and Aeolian volcanoes during 1865 are incredibly very similar to those occurred at the same volcanoes during 2002-2003.

\subsection{Concurrent activity during 126 BC}

Paulus Orosius, a Latin historian lived in the $5^{\text {th }}$ Century. In his Historiarum Adversus Paganos wrote: «Under the consulate of $\mathrm{M}$. Aemilius Laepidus and L. Orestes, Mount Etna was violently shaken by a powerful tremble and poured out waves of fire globes. The day after, the Lipari Islands (i.e., also known as Aeolian Islands) and nearby the sea reached such a high boiling point that rocks were burnt and broken up. Ships, axes, and wax were carbonized and melted, dead fishes were burnt on the sea surface, and several men, except those who were able to escape, were stifled and their inner organs burnt by breathing». Strabo, a Greek geographer, and Julius Obsequens, a Latin historian, had reported the same event in documents dated back to the $1^{\text {st }}$ Century BC (Geographia) and to the $4^{\text {th }}$ Century (De Prodigiis), respectively. The events reported by the above-cited authors refer to the explosive eruption of Mount Etna started in June $126 \mathrm{BC}$ and to the contemporaneous emissions of gas occurred in the offshore area between Vulcano and Panarea.

\section{Statistical analysis of the eruption catalogue}

This section analyses the historical frequency of simultaneous eruptions at two or three volcanoes among Etna, Stromboli, and Vulcano. The analysed catalogue is the one compiled by Simkin and Siebert (1994). We preferred to use the printed version of this catalogue (Simkin and Siebert, 1994) rather than the online version available at www.volcano.si.edu, because this latter is a non-static document updated in subsequent years (Siebert and Simkin, 2002), where, however, reasons for amendments are not explained, at least for what concerns the studied volcanoes. The problem of possible errors contained in the historical catalogue is addressed below in the discussion section.

As eruptions are often ongoing sequences of various and heterogeneous phenomena (see the preceding section), the problem studied here is simply whether or not eruptions at Etna, Stromboli, and Vulcano overlapped more often than expected by chance. This means that we do not consider eruption onset, whose exact dates are often lacking of poorly constrained (Simkin and Siebert, 1994). Instead, we analyse eruption durations, that is whether an eruption is in progress at some point in a specific time interval. To do so, simultaneous months and years including eruptions at two or three of the studied volcanoes are considered (tables III and IV). The analysis does not attempt to distinguish between eruptive styles or locations within a given volcanic system. This leaves the eruptive triggers poorly constrained, but makes the statistics possible for the historical data.

A null hypothesis can be constructed by using the observed record (tables III and IV) to calculate the expected probability of concurrent eruptions if the volcanoes do not interact. For instance, if the observed historical probability of Etna erupting in a given month or year $\left(p_{E}\right)$ is the number of months or years including Etna's eruptions divided by the total number of months or years included in the studied period, and the observed historical probability of Stromboli $\left(p_{S}\right)$ is computed in the same manner, then the expected historical probability (or ex- 
pected probability) of concurrent eruptions at Etna and Stromboli $p_{E-S}$ is simply the product $p_{E}$ $p_{S}$. In this section, the null hypothesis that eruptions at each of the volcanoes are statistically independent is evaluated by calculating the expected probability of concurrent eruptions (e.g., $\left.p_{E} p_{S}\right)$ at two or three volcanoes among Etna, Stromboli, and Vulcano and by comparing it to the observed probability of concurrent eruptions.

Before evaluating the probabilities, the catalogue is limited by type of data and by time to provide uniform completeness over the study interval. The catalogue is expected to be complete for the largest, most easily observable eruptions, but progressively more incomplete at the smaller sizes. Volcanic eruptions generally follow a power law distribution in sizes with many more small eruptions than large ones as quantified by VEI. Significant depressions at small VEI relative to the trend at large VEI suggest that there are missing eruptions in the catalogue. Figure 2 suggests that the volcanic catalogue used for this region (Simkin and Siebert, 1994 ) is only complete for about VEI $\geq 2$. For this reason, only eruptions with VEI $\geq 2$ are used for the analysis.

To find the temporal intervals significant for the statistical analysis, the temporal distribution of data (i.e., eruptions) for the studied volcanoes is analysed. The catalogue is significant, by number of data, since about the $18^{\text {th }}$ Century (fig. 3). About 1700 or younger times are therefore considered as the lower temporal boundary for the following statistical analysis.

The limited catalogue is used to estimate the probability of eruption per month and per year of each individual volcano as above explained. Results are plotted in probability versus time diagrams (fig. 4) to check whether the statistical analysis is sensitive to the time window. In each diagram, observed and expected probabilities of concurrent eruptions at two volcanoes between Etna, Stromboli, and Vulcano are compared. Etna-Vulcano and Stromboli-Vulcano pairs of volcanoes generally erupt simultaneously more frequently than would be expected based on the individual rates (figs. $4 \mathrm{~b}$ and $4 \mathrm{c}$ ). This relationship is particularly evident for the Stromboli-Vulcano pair (fig. 4c). In contrast, the simultaneous eruptions of Etna and Stromboli are often less frequent than would be expected based on the individual rates (fig. 4a).

The expected and observed probabilities of occurrence of simultaneous eruptions at the three studied volcanoes are also considered. Within the 1700-1890 period, the expected probability of a triple eruption is $0.015 \%$ by using months as a time period, and $0.400 \%$ using years. Within the same period (i.e., 1700-1890), the observed probability of a triple eruption is

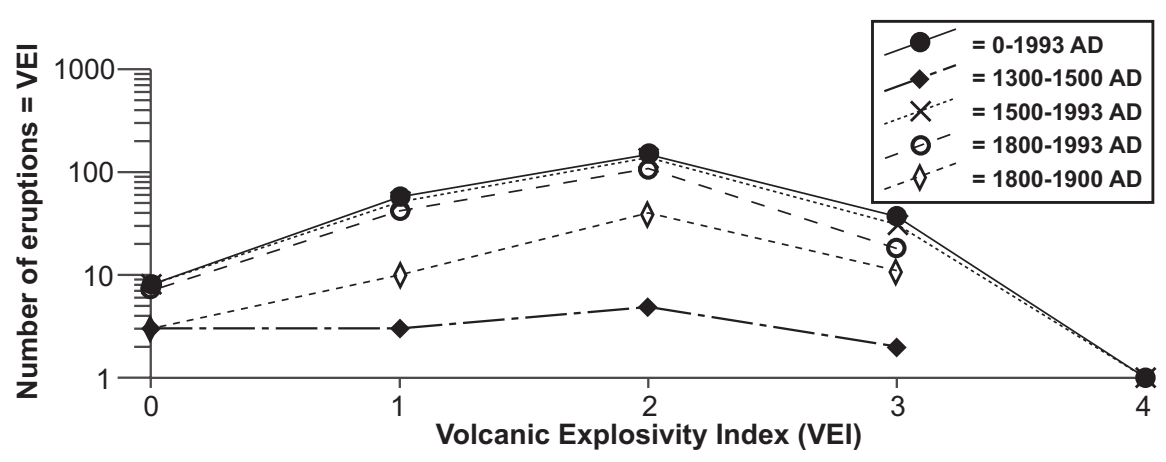

Fig. 2. Relationship between the number of eruptions and the «magnitude» of the eruption expressed by the volcanic explosivity index (VEI) for Etna, Stromboli, and Vulcano. Data (tables III and IV) are from Simkin and Siebert (1994). 


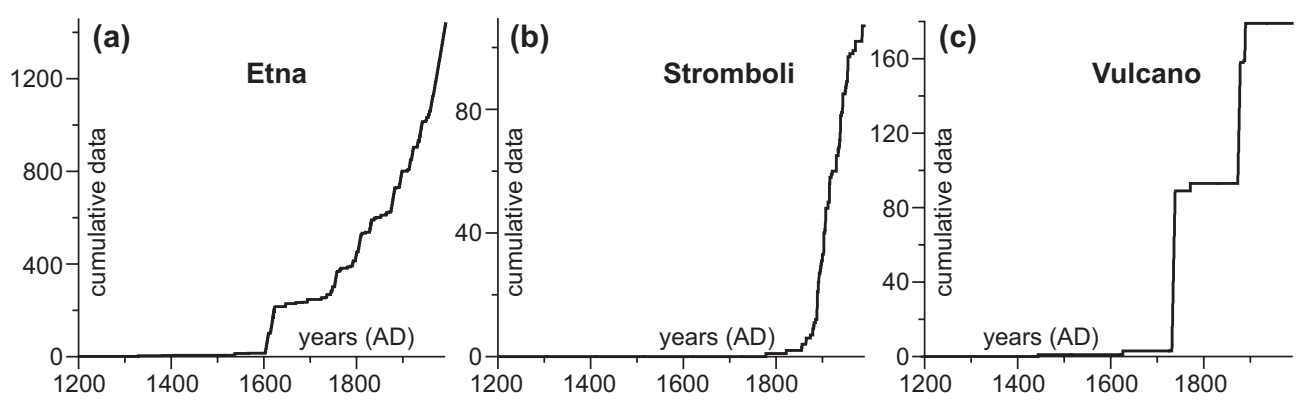

Fig. 3a-c. Relationships between the cumulative number of data (i.e., months including eruptions with VEI $\geq 2$ ) and time for (a) Etna, (b) Stromboli, and (c) Vulcano. Data (table III) are from Simkin and Siebert (1994).

Table II. Time order of erupting volcanoes in the occasion of duple and triple concurrent eruptions between 1700 and 1993. Data are from Simkin and Siebert (1994).

\begin{tabular}{llccc}
\hline \hline $\begin{array}{l}\text { type of concurrent } \\
\text { eruptions }\end{array}$ & $\begin{array}{l}\text { erupting } \\
\text { volcanoes }\end{array}$ & & \multicolumn{2}{c}{$\begin{array}{l}\text { no. of times in which the volcano erupted before } \\
\text { the others in theoccasion of concurrent eruptions }\end{array}$} \\
\cline { 2 - 4 } & Etna-Stromboli & Etna & Stromboli & Vulcano \\
\hline duple & 23 & 3 & $/$ \\
duple & Etna-Vulcano & 0 & $/$ & 4 \\
duple & Stromboli-Vulcano & $/$ & 0 & 2 \\
triple & Etna-Stromboli-Vulcano & 0 & 0 & 1 \\
\hline
\end{tabular}

$0.044 \%$ by using months as time period, and $1.047 \%$ by using years (tables III and IV). It follows that, in both cases, the observed probability is almost three times the expected probability.

It is eventually analysed whether concurrent eruptions often occur in a particular order. During the 1700-1993 period, the order of eruption is often Vulcano, Etna, and then Stromboli (tables II and III). If only two of the three erupt, the order is usually preserved.

\section{Statistical analysis of the earthquake catalogue}

To infer possible chronological correlations between the seismic energies released in the Et- nean and Aeolian areas, the related monthly totals of seismic moment $\left(\mathrm{TM}_{0}\right)$ are computed and compared.

The problem studied in this section is simply whether or not the greatest $\mathrm{T} M_{0}$-values for the Aeolian and Etnean areas overlapped more often than expected by chance. To do so, the magnitude of earthquakes (i.e., only those less than $30 \mathrm{~km}$ deep) occurred in the Aeolian and Etnean areas during the January 1983-December 2007 period (i.e., 300 months) is extracted from the INGV catalogue of earthquakes available on-line at www.ingv.it (Bollettino Sismico Italiano, Istituto Nazionale di Geofisica e Vulcanologia, Rome, Italy). Date and geographic location of earthquakes (i.e., Aeolian and Etnean areas) are included in the catalogue. The 

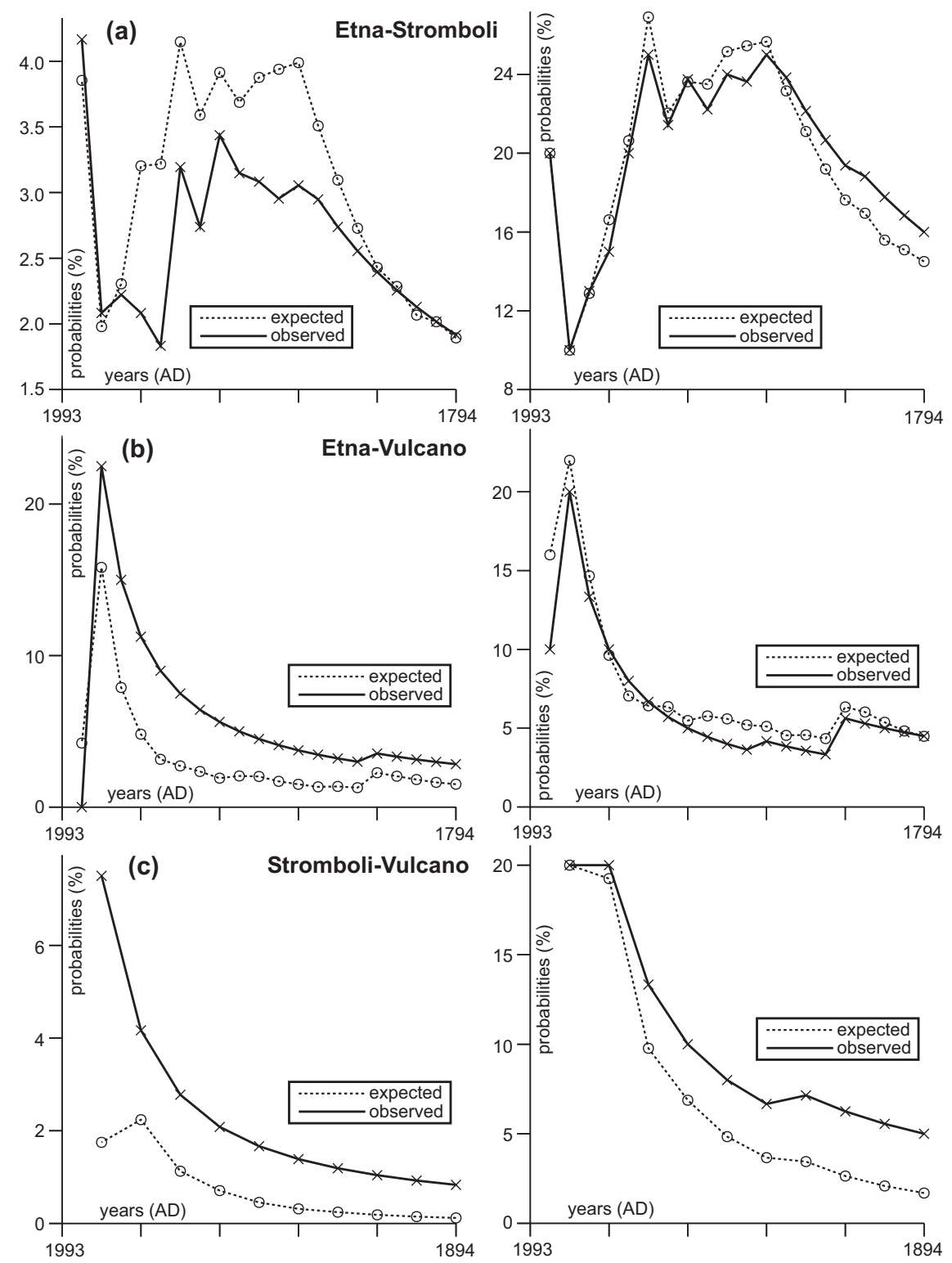

Fig. 4a-c. Expected and observed probabilities of occurrence of months (diagrams to the left) and years (diagrams to the right) including concurrent eruptions at (a) Etna and Stromboli, (b) Etna and Vulcano, and (c) Stromboli and Vulcano, versus time. Data (tables III and IV) are from Simkin and Siebert (1994). A rather high correlation of activity is observed in (b) and (c). 
magnitude of earthquakes is converted into the seismic moment by applying the equation $M=$ 2/3 $\log M_{0}-10.7$, where $M$ is the magnitude and $M_{0}$ is the seismic moment (Hanks and Kanamori, 1979). $\mathrm{T} M_{0}$-values are then computed (table V).

By following the same probabilistic approach used above for concurrent eruptions, the expected and observed probabilities that the greatest $\mathrm{TM}_{0}$ of the Aeolian and Etnean areas are contemporary are computed and compared (fig. 5). Figure 5 shows that, except for the two months with the greatest $\mathrm{TM}_{0}$-values (i.e., for no. of months $\geq 2$ in fig. 5), the observed probability is always significantly greater than the expected probability.

From the eruption catalogues of Simkin and Siebert (1994) and Siebert and Simkin (2002), it is inferred that, both for the Aeolian and Etnean areas, the greatest $\mathrm{T} M_{0}$-values are, in some cases, contemporary with or near to significant eruptions (e.g., October 1986, July 2001, October, 2002, and December, 2006 at Etna; November, 1994 and July. 2001 at Stromboli), whereas in some other cases there is no correlation with eruptions (e.g., December, 1997 and January, 1998 for Etna; October, 1986, July-August, 1995, and April, 2002 for Stromboli).

\section{Discussion}

The statistical analysis of the eruption catalogue shows that, during 1700-1993, concurrent eruptions at the studied volcanoes occurred, at least in some cases, at a frequency higher than expected based solely on random probability (fig. 4). This result, in particular, applies to the Stromboli-Vulcano and Etna-Vulcano pairs. It follows that concurrent eruptions at these volcanoes can be plausibly interpreted as non-accidental. The considered temporal interval is, however, limited and the statistical analyses may therefore be poorly representative of the real rate of concurrent eruptions. Moreover, statistical results may reflect reporting biases and the analysed catalogues may contain errors. These issues make our results preliminary and our hypotheses very speculative at least in some instances. On the other hand, statistical analyses do not consider eruptions with $\mathrm{VEI}<2$ and a series of volcanic phenomena such as degassing, unrest, or hydrothermalism, which are poorly reported in the catalogues but can be the manifestation of linked activities at Etna and Aeolian volcanoes (e.g., Cigolini et al., 2007). It follows that the incidence of concurrent volcanic phenomena at the studied volcanoes may be greater than that obtained by the statistical analyses presented in this paper.

The recent literature on eruption triggering suggests that the cause of concurrent eruptions may be a reciprocal stimulus or an external common one. Among the possible external causes, earthquakes are considered the most common and viable ones (e.g., Linde and Sacks, 1998; Manga and Brodsky, 2006). Earthquakes may trigger volcanic eruptions by dynamic stress (i.e., the shaking of rocks at the passage of seismic waves; e.g., Walter and Amelung, 2007). In addition to dynamic stress, fault slip induces a static stress change (i.e., the fault slip induces a permanent deformation in the nearby crust), which can be at the origin of volcanic eruptions (e.g., Bautista et al., 1996; Nostro et al., 1998). In the first case (i.e., dynamic stress), the earthquake and the volcano can even be very far (e.g., Manga and Brodsky, 2006). In contrast, the effect of the fault-related static stress change decays rapidly with the distance and, therefore, the fault has to be near the volcano to efficiently excite it.

In addition to earthquakes and fault slip, the stress changes induced by the annual sea-level variations (McNutt and Beavan, 1987), by the Earth and ocean daily tides (Glasby and Kasahara, 2001), the glacial loading (Tryggvasson, 1973), by the seasonal atmospheric variations (Ohtake and Nakahara, 1999), and by the regional tectonics (Mulargia et al., 1991; McNutt, 1999; Madonia et al., 2008) have been hypothesized as potential external triggers of volcanic eruptions.

Alternatively or complementarily, eruptions can reciprocally trigger other events. Either the static stress generated by the movement of magma (Miklius and Cervelli, 2003; Gudmundsson and Andrew, 2007) or the shaking generated by explosive eruptions and associat- 


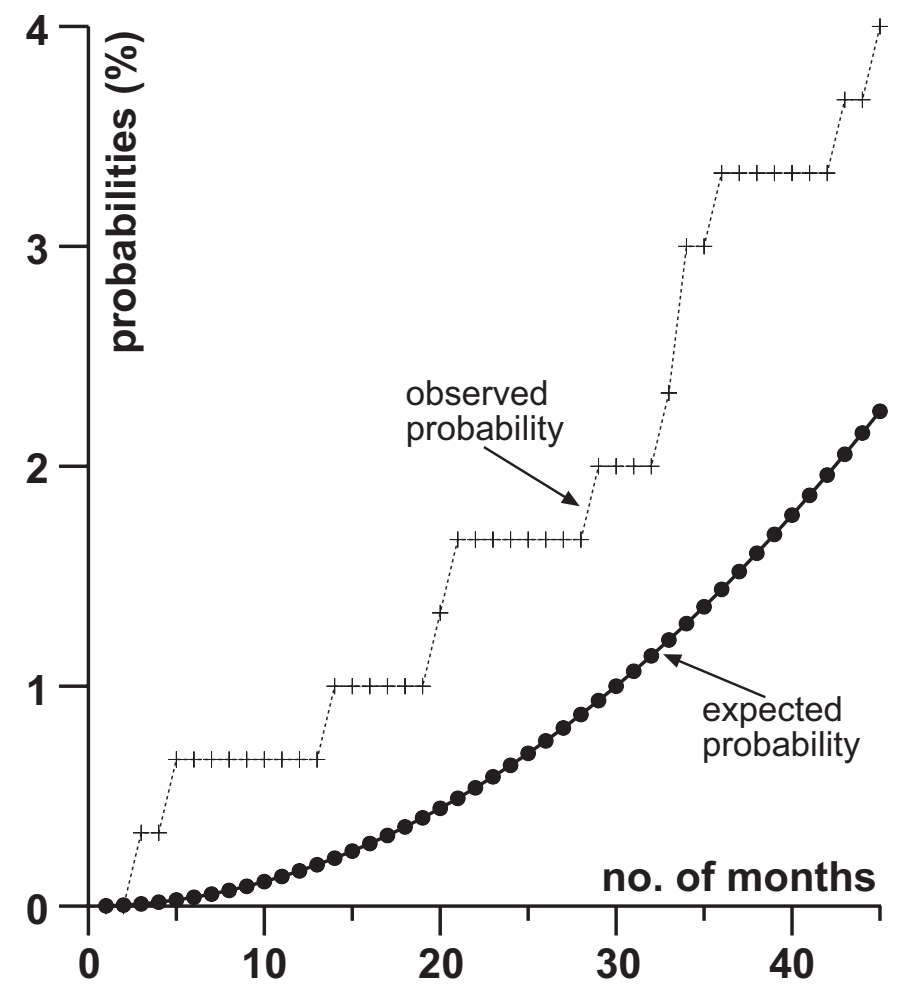

Fig. 5. Expected and observed probabilities of concurrence of months with the greatest monthly totals of seismic moment $\left(\mathrm{TM}_{0}\right)$ for the Aeolian and Etnean areas (table V). For a number of months greater than 45, the divergence between the expected and observed probabilities increases.

ed earthquakes (Kanamori and Givens, 1982) can trigger adjacent volcanoes.

Eventually, concurrent eruptions from adjacent volcanoes can be triggered by a simultaneous increase of magma supply from the mantle (Miklius and Cervelli, 2003).

The presented statistical and anecdotal evidence of concurrent activities at Etna, Stromboli, and Vulcano does not allow an unequivocal identification of a plausible triggering mechanism. It cannot even be discounted the idea that the triggering mechanism may have changed from time to time or may have been a combination of the above-quoted triggering mechanisms. Some simple inferences can, however, be drawn from the presented evidence. The sequential order of eruptions (table II) sug- gests that eruption-eruption reciprocal triggering may have been important. As Vulcano was often the most explosive of the volcanoes (Simkin and Siebert, 1994; tables III and IV), it likely generated the strongest seismic waves and hence usually began the sequence (table II). The vicinity of Vulcano to both Etna and Stromboli (fig. 1) may explain why its relationships with these volcanoes are seemingly causal (figs. 4b and 4c), whereas concurrent activities at Etna and Stromboli, which are as far as about $130 \mathrm{~km}$, seem accidental or less connected (fig. $4 \mathrm{a})$. The presence of an active fault zone between Vulcano and Etna (i.e., the Tindari Fault, fig. 1) may have enhanced the directivity of seismic waves between these volcanoes, thus promoting the dynamic stress transfer. Of 
course, whether or not a given dynamic input successfully triggers an eruption depends on the state of the volcano, particularly its magma chamber. Accordingly, it is not expected to observe perfect correlations or a deterministic sequence of eruptions.

A reciprocal triggering similar to that proposed for Iceland volcanoes (i.e., by direct mechanical interactions among volcanoes about 30 km distant; Gudmundsson and Andrew, 2007) seems less likely for the studied volcanoes, whose reciprocal distance is as small as $50 \mathrm{~km}$ (fig. 1). However, as shown by the reported anecdotal instances, the concurrent volcanic phenomena can be very heterogeneous and can involve other volcanoes in addition to Etna, Stromboli, and Vulcano. For instance, increased degassing from Panarea, which is only about $22 \mathrm{~km}$ from Stromboli and $30 \mathrm{~km}$ from Vulcano, may have been the consequence of direct mechanical interactions with the adjacent active volcanoes (e.g., Gudmundsson and Andrew, 2007).

Concerning external triggers, it is historically demonstrated that the studied volcanoes did not respond immediately or shortly to dynamic stresses generated by large nearby earthquakes (table I). Moreover, there is no evidence - anecdotal or otherwise - that either local or remote large earthquakes triggered eruptions in the region (see the NEIC Catalogue available on-line at neic.usgs.gov/neis/epic/ and the INGV Catalogue available on-line at www.ingv.it); however, also the hypothesis of a stimulus by external earthquakes cannot be fully discounted. For instance, the eruptive cycle of 2002-2003 involving eruptions at Etna and Stromboli and degassing phenomena at Panarea started about one month after the 6 September 2002, M 5.6, Palermo (fig. 1) earthquake. This sequence of seismic and volcanic phenomena has been interpreted as causal by Cigolini et al. (2007).

Alternatively, the concurrent eruptions at the Southern Italian volcanoes may have been the effect of the regional tectonic compression (fig. 1b; Goes et al., 2004; Neri et al., 2005). Such a stress may have been periodically relieved by a large earthquake on one segment of the regional fault network (Boschi et al., 1997; Neri et al., 2006) or, more often, by swarms of eruptions and moderate-to-small magnitude earthquakes where the triggering threshold is low (i.e., in the active volcanic districts) (e.g., Brodsky et al., 2000). The statistical analysis of earthquakes instrumentally-recorded between 1983 and 2007 (fig. 5) supports this hypothesis although, of course, coupled seismicity in the Etnean and Aeolian areas may alternatively be explained as the consequence of coupled volcanism triggered by some other cause such as a simultaneous increase of magma supply to Etna and the Aeolian volcanoes. A link between the regional tectonics and the volcanic activity of Etna was previously proposed by Mulargia et al. (1991). Moreover, Feuillet et al. (2006) demonstrated that the largest nearby earthquakes occurred after years-long-periods of volcanic activity of Etna, this evidence possibly indicating a period of tectonic load that first triggered the volcanic activity and, eventually, a large earthquake.

\section{Conclusions}

Analyses of eruption and earthquake catalogues show that causal links between concurrent eruptions at two or three volcanoes among Etna, Stromboli, and Vulcano are plausible, particularly in the Stromboli-Vulcano and EtnaVulcano cases. These links are also supported by recent and historical anecdotal instances. Limits of statistical results, however, compel further research to better constrain the temporal pattern of such phenomena and to identify their causes. We look forward to our predictions of a moderate incidence of concurrent eruptions being confirmed or refuted with the passage of time and with more sophisticated analyses of new events (e.g., Jaquet and Carniel, 2006).

\section{Acknowledgements}

F. Barberi, M. Bebbington, E. Brodsky, A. Gudmundsson, C. Jaupart, O. Jaquet, D. Peacock, and S. Tavani are thanked for useful comments and suggestions. L. Da Riva helped with Latin translation from the Historiarum Adversus Paganos by Paulus Orosius. 
Table III. Eruption (with VEI $\geq 2$ ) occurrences by month between 0 and 1993 AD at Etna, Stromboli, and Vulcano.

VEI $=$ Volcanic Explosivity Index; $0=$ no occurrence; 1 = occurrence. Data are from Simkin and Siebert (1994).

\begin{tabular}{|c|c|c|c|c|c|c|}
\hline year & month & $\begin{array}{c}\text { Etna } \\
\text { eruptions }\end{array}$ & VEI & $\begin{array}{l}\text { Stromboli } \\
\text { eruptions }\end{array}$ & VEI & $\begin{array}{l}\text { Vulcano } \\
\text { eruptions }\end{array}$ \\
\hline 252 & 2 & 1 & 3 & 0 & & 0 \\
\hline 1329 & 6 & 1 & 2 & 0 & & 0 \\
\hline 1329 & 7 & 1 & 3 & 0 & & 0 \\
\hline 1329 & 8 & 1 & 3 & 0 & & 0 \\
\hline 1381 & 8 & 1 & 2 & 0 & & 0 \\
\hline 1408 & 11 & 1 & 3 & 0 & & 0 \\
\hline 1444 & 2 & 0 & & 0 & & 1 \\
\hline 1536 & 3 & 1 & 3 & 0 & & 0 \\
\hline 1536 & 4 & 1 & 3 & 0 & & 0 \\
\hline 1537 & 3 & 1 & 2 & 0 & & 0 \\
\hline 1537 & 4 & 1 & 2 & 0 & & 0 \\
\hline 1537 & 5 & 1 & 2 & 0 & & 0 \\
\hline 1537 & 6 & 1 & 2 & 0 & & 0 \\
\hline 1537 & 7 & 1 & 2 & 0 & & 0 \\
\hline 1541 & 7 & 1 & 2 & 0 & & 0 \\
\hline 1566 & 11 & 1 & 2 & 0 & & 0 \\
\hline 1603 & 7 & 1 & 2 & 0 & & 0 \\
\hline 1603 & 8 & 1 & 2 & 0 & & 0 \\
\hline 1603 & 9 & 1 & 2 & 0 & & 0 \\
\hline 1603 & 10 & 1 & 2 & 0 & & 0 \\
\hline 1603 & 11 & 1 & 2 & 0 & & 0 \\
\hline 1603 & 12 & 1 & 2 & 0 & & 0 \\
\hline 1604 & 1 & 1 & 2 & 0 & & 0 \\
\hline 1604 & 2 & 1 & 2 & 0 & & 0 \\
\hline 1604 & 3 & 1 & 2 & 0 & & 0 \\
\hline 1604 & 4 & 1 & 2 & 0 & & 0 \\
\hline 1604 & 5 & 1 & 2 & 0 & & 0 \\
\hline 1604 & 6 & 1 & 2 & 0 & & 0 \\
\hline 1604 & 7 & 1 & 2 & 0 & & 0 \\
\hline 1604 & 8 & 1 & 2 & 0 & & 0 \\
\hline 1604 & 9 & 1 & 2 & 0 & & 0 \\
\hline 1604 & 10 & 1 & 2 & 0 & & 0 \\
\hline 1604 & 11 & 1 & 2 & 0 & & 0 \\
\hline 1604 & 12 & 1 & 2 & 0 & & 0 \\
\hline 1605 & 1 & 1 & 2 & 0 & & 0 \\
\hline 1605 & 2 & 1 & 2 & 0 & & 0 \\
\hline 1605 & 3 & 1 & 2 & 0 & & 0 \\
\hline 1605 & 4 & 1 & 2 & 0 & & 0 \\
\hline
\end{tabular}


Table III. (continued).

\begin{tabular}{|c|c|c|c|c|c|c|}
\hline year & month & $\begin{array}{c}\text { Etna } \\
\text { eruptions }\end{array}$ & VEI & $\begin{array}{l}\text { Stromboli } \\
\text { eruptions }\end{array}$ & VEI & $\begin{array}{c}\text { Vulcano } \\
\text { eruptions }\end{array}$ \\
\hline 1605 & 5 & 1 & 2 & 0 & & 0 \\
\hline 1605 & 6 & 1 & 2 & 0 & & 0 \\
\hline 1605 & 7 & 1 & 2 & 0 & & 0 \\
\hline 1605 & 8 & 1 & 2 & 0 & & 0 \\
\hline 1605 & 9 & 1 & 2 & 0 & & 0 \\
\hline 1605 & 10 & 1 & 2 & 0 & & 0 \\
\hline 1605 & 11 & 1 & 2 & 0 & & 0 \\
\hline 1605 & 12 & 1 & 2 & 0 & & 0 \\
\hline 1606 & 1 & 1 & 2 & 0 & & 0 \\
\hline 1606 & 2 & 1 & 2 & 0 & & 0 \\
\hline 1606 & 3 & 1 & 2 & 0 & & 0 \\
\hline 1606 & 4 & 1 & 2 & 0 & & 0 \\
\hline 1606 & 5 & 1 & 2 & 0 & & 0 \\
\hline 1606 & 6 & 1 & 2 & 0 & & 0 \\
\hline 1606 & 7 & 1 & 2 & 0 & & 0 \\
\hline 1606 & 8 & 1 & 2 & 0 & & 0 \\
\hline 1606 & 9 & 1 & 2 & 0 & & 0 \\
\hline 1606 & 10 & 1 & 2 & 0 & & 0 \\
\hline 1606 & 11 & 1 & 2 & 0 & & 0 \\
\hline 1606 & 12 & 1 & 2 & 0 & & 0 \\
\hline 1607 & 1 & 1 & 2 & 0 & & 0 \\
\hline 1607 & 2 & 1 & 2 & 0 & & 0 \\
\hline 1607 & 3 & 1 & 2 & 0 & & 0 \\
\hline 1607 & 4 & 1 & 2 & 0 & & 0 \\
\hline 1607 & 5 & 1 & 2 & 0 & & 0 \\
\hline 1607 & 6 & 1 & 2 & 0 & & 0 \\
\hline 1607 & 7 & 1 & 2 & 0 & & 0 \\
\hline 1607 & 8 & 1 & 2 & 0 & & 0 \\
\hline 1607 & 9 & 1 & 2 & 0 & & 0 \\
\hline 1607 & 10 & 1 & 2 & 0 & & 0 \\
\hline 1607 & 11 & 1 & 2 & 0 & & 0 \\
\hline 1607 & 12 & 1 & 2 & 0 & & 0 \\
\hline 1608 & 1 & 1 & 2 & 0 & & 0 \\
\hline 1608 & 2 & 1 & 2 & 0 & & 0 \\
\hline 1608 & 3 & 1 & 2 & 0 & & 0 \\
\hline 1608 & 4 & 1 & 2 & 0 & & 0 \\
\hline 1608 & 5 & 1 & 2 & 0 & & 0 \\
\hline 1608 & 6 & 1 & 2 & 0 & & 0 \\
\hline 1608 & 7 & 1 & 2 & 0 & & 0 \\
\hline 1608 & 8 & 1 & 2 & 0 & & 0 \\
\hline
\end{tabular}


Table III. (continued).

\begin{tabular}{|c|c|c|c|c|c|c|}
\hline year & month & $\begin{array}{c}\text { Etna } \\
\text { eruptions }\end{array}$ & VEI & $\begin{array}{l}\text { Stromboli } \\
\text { eruptions }\end{array}$ & VEI & $\begin{array}{c}\text { Vulcano } \\
\text { eruptions }\end{array}$ \\
\hline 1608 & 9 & 1 & 2 & 0 & & 0 \\
\hline 1608 & 10 & 1 & 2 & 0 & & 0 \\
\hline 1608 & 11 & 1 & 2 & 0 & & 0 \\
\hline 1608 & 12 & 1 & 2 & 0 & & 0 \\
\hline 1609 & 1 & 1 & 2 & 0 & & 0 \\
\hline 1609 & 2 & 1 & 2 & 0 & & 0 \\
\hline 1609 & 3 & 1 & 2 & 0 & & 0 \\
\hline 1609 & 4 & 1 & 2 & 0 & & 0 \\
\hline 1609 & 5 & 1 & 2 & 0 & & 0 \\
\hline 1609 & 6 & 1 & 2 & 0 & & 0 \\
\hline 1609 & 7 & 1 & 2 & 0 & & 0 \\
\hline 1609 & 8 & 1 & 2 & 0 & & 0 \\
\hline 1609 & 9 & 1 & 2 & 0 & & 0 \\
\hline 1609 & 10 & 1 & 2 & 0 & & 0 \\
\hline 1609 & 11 & 1 & 2 & 0 & & 0 \\
\hline 1609 & 12 & 1 & 2 & 0 & & 0 \\
\hline 1610 & 1 & 1 & 2 & 0 & & 0 \\
\hline 1610 & 2 & 1 & 2 & 0 & & 0 \\
\hline 1610 & 3 & 1 & 2 & 0 & & 0 \\
\hline 1610 & 4 & 1 & 2 & 0 & & 0 \\
\hline 1610 & 5 & 1 & 2 & 0 & & 0 \\
\hline 1610 & 6 & 1 & 2 & 0 & & 0 \\
\hline 1610 & 7 & 1 & 2 & 0 & & 0 \\
\hline 1610 & 8 & 1 & 2 & 0 & & 0 \\
\hline 1614 & 7 & 1 & 2 & 0 & & 0 \\
\hline 1614 & 8 & 1 & 2 & 0 & & 0 \\
\hline 1614 & 9 & 1 & 2 & 0 & & 0 \\
\hline 1614 & 10 & 1 & 2 & 0 & & 0 \\
\hline 1614 & 11 & 1 & 2 & 0 & & 0 \\
\hline 1614 & 12 & 1 & 2 & 0 & & 0 \\
\hline 1615 & 1 & 1 & 2 & 0 & & 0 \\
\hline 1615 & 2 & 1 & 2 & 0 & & 0 \\
\hline 1615 & 3 & 1 & 2 & 0 & & 0 \\
\hline 1615 & 4 & 1 & 2 & 0 & & 0 \\
\hline 1615 & 5 & 1 & 2 & 0 & & 0 \\
\hline 1615 & 6 & 1 & 2 & 0 & & 0 \\
\hline 1615 & 7 & 1 & 2 & 0 & & 0 \\
\hline 1615 & 8 & 1 & 2 & 0 & & 0 \\
\hline 1615 & 9 & 1 & 2 & 0 & & 0 \\
\hline 1615 & 10 & 1 & 2 & 0 & & 0 \\
\hline
\end{tabular}


Table III. (continued).

\begin{tabular}{|c|c|c|c|c|c|c|}
\hline year & month & $\begin{array}{c}\text { Etna } \\
\text { eruptions }\end{array}$ & VEI & $\begin{array}{l}\text { Stromboli } \\
\text { eruptions }\end{array}$ & VEI & $\begin{array}{c}\text { Vulcano } \\
\text { eruptions }\end{array}$ \\
\hline 1615 & 11 & 1 & 2 & 0 & & 0 \\
\hline 1615 & 12 & 1 & 2 & 0 & & 0 \\
\hline 1616 & 1 & 1 & 2 & 0 & & 0 \\
\hline 1616 & 2 & 1 & 2 & 0 & & 0 \\
\hline 1616 & 3 & 1 & 2 & 0 & & 0 \\
\hline 1616 & 4 & 1 & 2 & 0 & & 0 \\
\hline 1616 & 5 & 1 & 2 & 0 & & 0 \\
\hline 1616 & 6 & 1 & 2 & 0 & & 0 \\
\hline 1616 & 7 & 1 & 2 & 0 & & 0 \\
\hline 1616 & 8 & 1 & 2 & 0 & & 0 \\
\hline 1616 & 9 & 1 & 2 & 0 & & 0 \\
\hline 1616 & 10 & 1 & 2 & 0 & & 0 \\
\hline 1616 & 11 & 1 & 2 & 0 & & 0 \\
\hline 1616 & 12 & 1 & 2 & 0 & & 0 \\
\hline 1617 & 1 & 1 & 2 & 0 & & 0 \\
\hline 1617 & 2 & 1 & 2 & 0 & & 0 \\
\hline 1617 & 3 & 1 & 2 & 0 & & 0 \\
\hline 1617 & 4 & 1 & 2 & 0 & & 0 \\
\hline 1617 & 5 & 1 & 2 & 0 & & 0 \\
\hline 1617 & 6 & 1 & 2 & 0 & & 0 \\
\hline 1617 & 7 & 1 & 2 & 0 & & 0 \\
\hline 1617 & 8 & 1 & 2 & 0 & & 0 \\
\hline 1617 & 9 & 1 & 2 & 0 & & 0 \\
\hline 1617 & 10 & 1 & 2 & 0 & & 0 \\
\hline 1617 & 11 & 1 & 2 & 0 & & 0 \\
\hline 1617 & 12 & 1 & 2 & 0 & & 0 \\
\hline 1618 & 1 & 1 & 2 & 0 & & 0 \\
\hline 1618 & 2 & 1 & 2 & 0 & & 0 \\
\hline 1618 & 3 & 1 & 2 & 0 & & 0 \\
\hline 1618 & 4 & 1 & 2 & 0 & & 0 \\
\hline 1618 & 5 & 1 & 2 & 0 & & 0 \\
\hline 1618 & 6 & 1 & 2 & 0 & & 0 \\
\hline 1618 & 7 & 1 & 2 & 0 & & 0 \\
\hline 1618 & 8 & 1 & 2 & 0 & & 0 \\
\hline 1618 & 9 & 1 & 2 & 0 & & 0 \\
\hline 1618 & 10 & 1 & 2 & 0 & & 0 \\
\hline 1618 & 11 & 1 & 2 & 0 & & 0 \\
\hline 1618 & 12 & 1 & 2 & 0 & & 0 \\
\hline 1619 & 1 & 1 & 2 & 0 & & 0 \\
\hline 1619 & 2 & 1 & 2 & 0 & & 0 \\
\hline
\end{tabular}


Table III. (continued).

\begin{tabular}{|c|c|c|c|c|c|c|}
\hline year & month & $\begin{array}{c}\text { Etna } \\
\text { eruptions }\end{array}$ & VEI & $\begin{array}{l}\text { Stromboli } \\
\text { eruptions }\end{array}$ & VEI & $\begin{array}{c}\text { Vulcano } \\
\text { eruptions }\end{array}$ \\
\hline 1619 & 3 & 1 & 2 & 0 & & 0 \\
\hline 1619 & 4 & 1 & 2 & 0 & & 0 \\
\hline 1619 & 5 & 1 & 2 & 0 & & 0 \\
\hline 1619 & 6 & 1 & 2 & 0 & & 0 \\
\hline 1619 & 7 & 1 & 2 & 0 & & 0 \\
\hline 1619 & 8 & 1 & 2 & 0 & & 0 \\
\hline 1619 & 9 & 1 & 2 & 0 & & 0 \\
\hline 1619 & 10 & 1 & 2 & 0 & & 0 \\
\hline 1619 & 11 & 1 & 2 & 0 & & 0 \\
\hline 1619 & 12 & 1 & 2 & 0 & & 0 \\
\hline 1620 & 1 & 1 & 2 & 0 & & 0 \\
\hline 1620 & 2 & 1 & 2 & 0 & & 0 \\
\hline 1620 & 3 & 1 & 2 & 0 & & 0 \\
\hline 1620 & 4 & 1 & 2 & 0 & & 0 \\
\hline 1620 & 5 & 1 & 2 & 0 & & 0 \\
\hline 1620 & 6 & 1 & 2 & 0 & & 0 \\
\hline 1620 & 7 & 1 & 2 & 0 & & 0 \\
\hline 1620 & 8 & 1 & 2 & 0 & & 0 \\
\hline 1620 & 9 & 1 & 2 & 0 & & 0 \\
\hline 1620 & 10 & 1 & 2 & 0 & & 0 \\
\hline 1620 & 11 & 1 & 2 & 0 & & 0 \\
\hline 1620 & 12 & 1 & 2 & 0 & & 0 \\
\hline 1621 & 1 & 1 & 2 & 0 & & 0 \\
\hline 1621 & 2 & 1 & 2 & 0 & & 0 \\
\hline 1621 & 3 & 1 & 2 & 0 & & 0 \\
\hline 1621 & 4 & 1 & 2 & 0 & & 0 \\
\hline 1621 & 5 & 1 & 2 & 0 & & 0 \\
\hline 1621 & 6 & 1 & 2 & 0 & & 0 \\
\hline 1621 & 7 & 1 & 2 & 0 & & 0 \\
\hline 1621 & 8 & 1 & 2 & 0 & & 0 \\
\hline 1621 & 9 & 1 & 2 & 0 & & 0 \\
\hline 1621 & 10 & 1 & 2 & 0 & & 0 \\
\hline 1621 & 11 & 1 & 2 & 0 & & 0 \\
\hline 1621 & 12 & 1 & 2 & 0 & & 0 \\
\hline 1622 & 1 & 1 & 2 & 0 & & 0 \\
\hline 1622 & 2 & 1 & 2 & 0 & & 0 \\
\hline 1622 & 3 & 1 & 2 & 0 & & 0 \\
\hline 1622 & 4 & 1 & 2 & 0 & & 0 \\
\hline 1622 & 5 & 1 & 2 & 0 & & 0 \\
\hline 1622 & 6 & 1 & 2 & 0 & & 0 \\
\hline
\end{tabular}


Table III. (continued).

\begin{tabular}{|c|c|c|c|c|c|c|}
\hline year & month & $\begin{array}{c}\text { Etna } \\
\text { eruptions }\end{array}$ & VEI & $\begin{array}{l}\text { Stromboli } \\
\text { eruptions }\end{array}$ & VEI & $\begin{array}{c}\text { Vulcano } \\
\text { eruptions }\end{array}$ \\
\hline 1622 & 7 & 1 & 2 & 0 & & 0 \\
\hline 1622 & 8 & 1 & 2 & 0 & & 0 \\
\hline 1622 & 9 & 1 & 2 & 0 & & 0 \\
\hline 1622 & 10 & 1 & 2 & 0 & & 0 \\
\hline 1622 & 11 & 1 & 2 & 0 & & 0 \\
\hline 1622 & 12 & 1 & 2 & 0 & & 0 \\
\hline 1623 & 1 & 1 & 2 & 0 & & 0 \\
\hline 1623 & 2 & 1 & 2 & 0 & & 0 \\
\hline 1623 & 3 & 1 & 2 & 0 & & 0 \\
\hline 1623 & 4 & 1 & 2 & 0 & & 0 \\
\hline 1623 & 5 & 1 & 2 & 0 & & 0 \\
\hline 1623 & 6 & 1 & 2 & 0 & & 0 \\
\hline 1623 & 7 & 1 & 2 & 0 & & 0 \\
\hline 1623 & 8 & 1 & 2 & 0 & & 0 \\
\hline 1623 & 9 & 1 & 2 & 0 & & 0 \\
\hline 1623 & 10 & 1 & 2 & 0 & & 0 \\
\hline 1623 & 11 & 1 & 2 & 0 & & 0 \\
\hline 1623 & 12 & 1 & 2 & 0 & & 0 \\
\hline 1624 & 1 & 1 & 2 & 0 & & 0 \\
\hline 1626 & 3 & 0 & & 0 & & 1 \\
\hline 1626 & 4 & 0 & & 0 & & 1 \\
\hline 1646 & 11 & 1 & 2 & 0 & & 0 \\
\hline 1646 & 12 & 1 & 2 & 0 & & 0 \\
\hline 1647 & 1 & 1 & 2 & 0 & & 0 \\
\hline 1647 & 2 & 1 & 2 & 0 & & 0 \\
\hline 1647 & 3 & 1 & 2 & 0 & & 0 \\
\hline 1647 & 4 & 1 & 2 & 0 & & 0 \\
\hline 1647 & 5 & 1 & 2 & 0 & & 0 \\
\hline 1647 & 6 & 1 & 2 & 0 & & 0 \\
\hline 1647 & 7 & 1 & 2 & 0 & & 0 \\
\hline 1647 & 8 & 1 & 2 & 0 & & 0 \\
\hline 1647 & 9 & 1 & 2 & 0 & & 0 \\
\hline 1647 & 10 & 1 & 2 & 0 & & 0 \\
\hline 1647 & 11 & 1 & 2 & 0 & & 0 \\
\hline 1669 & 3 & 1 & 3 & 0 & & 0 \\
\hline 1669 & 4 & 1 & 3 & 0 & & 0 \\
\hline 1669 & 5 & 1 & 3 & 0 & & 0 \\
\hline 1669 & 6 & 1 & 3 & 0 & & 0 \\
\hline 1669 & 7 & 1 & 3 & 0 & & 0 \\
\hline 1682 & 9 & 1 & 2 & 0 & & 0 \\
\hline
\end{tabular}


Table III. (continued).

\begin{tabular}{|c|c|c|c|c|c|c|}
\hline year & month & $\begin{array}{c}\text { Etna } \\
\text { eruptions }\end{array}$ & VEI & $\begin{array}{l}\text { Stromboli } \\
\text { eruptions }\end{array}$ & VEI & $\begin{array}{c}\text { Vulcano } \\
\text { eruptions }\end{array}$ \\
\hline 1693 & 12 & 1 & 3 & 0 & & 0 \\
\hline 1694 & 1 & 1 & 3 & 0 & & 0 \\
\hline 1694 & 2 & 1 & 3 & 0 & & 0 \\
\hline 1694 & 3 & 1 & 3 & 0 & & 0 \\
\hline 1694 & 4 & 1 & 3 & 0 & & 0 \\
\hline 1694 & 5 & 1 & 3 & 0 & & 0 \\
\hline 1694 & 6 & 1 & 3 & 0 & & 0 \\
\hline 1694 & 7 & 1 & 3 & 0 & & 0 \\
\hline 1694 & 8 & 1 & 3 & 0 & & 0 \\
\hline 1694 & 9 & 1 & 3 & 0 & & 0 \\
\hline 1694 & 10 & 1 & 3 & 0 & & 0 \\
\hline 1694 & 11 & 1 & 3 & 0 & & 0 \\
\hline 1723 & 11 & 1 & 2 & 0 & & 0 \\
\hline 1723 & 12 & 1 & 2 & 0 & & 0 \\
\hline 1724 & 1 & 1 & 2 & 0 & & 0 \\
\hline 1724 & 2 & 1 & 2 & 0 & & 0 \\
\hline 1724 & 3 & 1 & 2 & 0 & & 0 \\
\hline 1724 & 4 & 1 & 2 & 0 & & 0 \\
\hline 1724 & 5 & 1 & 2 & 0 & & 0 \\
\hline 1731 & 12 & 0 & & 0 & & 1 \\
\hline 1732 & 1 & 0 & & 0 & & 1 \\
\hline 1732 & 2 & 0 & & 0 & & 1 \\
\hline 1732 & 3 & 0 & & 0 & & 1 \\
\hline 1732 & 4 & 0 & & 0 & & 1 \\
\hline 1732 & 5 & 0 & & 0 & & 1 \\
\hline 1732 & 6 & 0 & & 0 & & 1 \\
\hline 1732 & 7 & 0 & & 0 & & 1 \\
\hline 1732 & 8 & 0 & & 0 & & 1 \\
\hline 1732 & 9 & 0 & & 0 & & 1 \\
\hline 1732 & 10 & 0 & & 0 & & 1 \\
\hline 1732 & 11 & 0 & & 0 & & 1 \\
\hline 1732 & 12 & 1 & 2 & 0 & & 1 \\
\hline 1733 & 1 & 1 & 2 & 0 & & 1 \\
\hline 1733 & 2 & 0 & & 0 & & 1 \\
\hline 1733 & 3 & 0 & & 0 & & 1 \\
\hline 1733 & 4 & 0 & & 0 & & 1 \\
\hline 1733 & 5 & 0 & & 0 & & 1 \\
\hline 1733 & 6 & 0 & & 0 & & 1 \\
\hline 1733 & 7 & 0 & & 0 & & 1 \\
\hline 1733 & 8 & 0 & & 0 & & 1 \\
\hline
\end{tabular}


Table III. (continued).

\begin{tabular}{|c|c|c|c|c|c|c|}
\hline year & month & $\begin{array}{c}\text { Etna } \\
\text { eruptions }\end{array}$ & VEI & $\begin{array}{l}\text { Stromboli } \\
\text { eruptions }\end{array}$ & VEI & $\begin{array}{c}\text { Vulcano } \\
\text { eruptions }\end{array}$ \\
\hline 1733 & 9 & 0 & & 0 & & 1 \\
\hline 1733 & 10 & 0 & & 0 & & 1 \\
\hline 1733 & 11 & 0 & & 0 & & 1 \\
\hline 1733 & 12 & 0 & & 0 & & 1 \\
\hline 1734 & 1 & 0 & & 0 & & 1 \\
\hline 1734 & 2 & 0 & & 0 & & 1 \\
\hline 1734 & 3 & 0 & & 0 & & 1 \\
\hline 1734 & 4 & 0 & & 0 & & 1 \\
\hline 1734 & 5 & 0 & & 0 & & 1 \\
\hline 1734 & 6 & 0 & & 0 & & 1 \\
\hline 1734 & 7 & 0 & & 0 & & 1 \\
\hline 1734 & 8 & 0 & & 0 & & 1 \\
\hline 1734 & 9 & 0 & & 0 & & 1 \\
\hline 1734 & 10 & 0 & & 0 & & 1 \\
\hline 1734 & 11 & 0 & & 0 & & 1 \\
\hline 1734 & 12 & 0 & & 0 & & 1 \\
\hline 1735 & 1 & 0 & & 0 & & 1 \\
\hline 1735 & 2 & 0 & & 0 & & 1 \\
\hline 1735 & 3 & 0 & & 0 & & 1 \\
\hline 1735 & 4 & 0 & & 0 & & 1 \\
\hline 1735 & 5 & 0 & & 0 & & 1 \\
\hline 1735 & 6 & 0 & & 0 & & 1 \\
\hline 1735 & 7 & 0 & & 0 & & 1 \\
\hline 1735 & 8 & 0 & & 0 & & 1 \\
\hline 1735 & 9 & 0 & & 0 & & 1 \\
\hline 1735 & 10 & 1 & 2 & 0 & & 1 \\
\hline 1735 & 11 & 1 & 2 & 0 & & 1 \\
\hline 1735 & 12 & 1 & 2 & 0 & & 1 \\
\hline 1736 & 1 & 1 & 2 & 0 & & 1 \\
\hline 1736 & 2 & 1 & 2 & 0 & & 1 \\
\hline 1736 & 3 & 1 & 2 & 0 & & 1 \\
\hline 1736 & 4 & 1 & 2 & 0 & & 1 \\
\hline 1736 & 5 & 1 & 2 & 0 & & 1 \\
\hline 1736 & 6 & 1 & 2 & 0 & & 1 \\
\hline 1736 & 7 & 1 & 2 & 0 & & 1 \\
\hline 1736 & 8 & 1 & 2 & 0 & & 1 \\
\hline 1736 & 9 & 1 & 2 & 0 & & 1 \\
\hline 1736 & 10 & 0 & & 0 & & 1 \\
\hline 1736 & 11 & 0 & & 0 & & 1 \\
\hline 1736 & 12 & 0 & & 0 & & 1 \\
\hline
\end{tabular}


Table III. (continued).

\begin{tabular}{|c|c|c|c|c|c|c|}
\hline year & month & $\begin{array}{c}\text { Etna } \\
\text { eruptions }\end{array}$ & VEI & $\begin{array}{l}\text { Stromboli } \\
\text { eruptions }\end{array}$ & VEI & $\begin{array}{c}\text { Vulcano } \\
\text { eruptions }\end{array}$ \\
\hline 1737 & 1 & 0 & & 0 & & 1 \\
\hline 1737 & 2 & 0 & & 0 & & 1 \\
\hline 1737 & 3 & 0 & & 0 & & 1 \\
\hline 1737 & 4 & 0 & & 0 & & 1 \\
\hline 1737 & 5 & 0 & & 0 & & 1 \\
\hline 1737 & 6 & 0 & & 0 & & 1 \\
\hline 1737 & 7 & 0 & & 0 & & 1 \\
\hline 1737 & 8 & 0 & & 0 & & 1 \\
\hline 1737 & 9 & 0 & & 0 & & 1 \\
\hline 1737 & 10 & 0 & & 0 & & 1 \\
\hline 1737 & 11 & 0 & & 0 & & 1 \\
\hline 1737 & 12 & 0 & & 0 & & 1 \\
\hline 1738 & 1 & 0 & & 0 & & 1 \\
\hline 1738 & 2 & 0 & & 0 & & 1 \\
\hline 1738 & 3 & 0 & & 0 & & 1 \\
\hline 1738 & 4 & 0 & & 0 & & 1 \\
\hline 1738 & 5 & 0 & & 0 & & 1 \\
\hline 1738 & 6 & 0 & & 0 & & 1 \\
\hline 1738 & 7 & 0 & & 0 & & 1 \\
\hline 1738 & 8 & 0 & & 0 & & 1 \\
\hline 1738 & 9 & 0 & & 0 & & 1 \\
\hline 1738 & 10 & 0 & & 0 & & 1 \\
\hline 1738 & 11 & 0 & & 0 & & 1 \\
\hline 1738 & 12 & 0 & & 0 & & 1 \\
\hline 1739 & 1 & 0 & & 0 & & 1 \\
\hline 1744 & 7 & 1 & 2 & 0 & & 0 \\
\hline 1744 & 8 & 1 & 2 & 0 & & 0 \\
\hline 1744 & 9 & 1 & 2 & 0 & & 0 \\
\hline 1744 & 10 & 1 & 2 & 0 & & 0 \\
\hline 1744 & 11 & 1 & 2 & 0 & & 0 \\
\hline 1744 & 12 & 1 & 2 & 0 & & 0 \\
\hline 1745 & 1 & 1 & 2 & 0 & & 0 \\
\hline 1745 & 2 & 1 & 2 & 0 & & 0 \\
\hline 1745 & 3 & 1 & 2 & 0 & & 0 \\
\hline 1745 & 4 & 1 & 2 & 0 & & 0 \\
\hline 1745 & 5 & 1 & 2 & 0 & & 0 \\
\hline 1745 & 6 & 1 & 2 & 0 & & 0 \\
\hline 1745 & 7 & 1 & 2 & 0 & & 0 \\
\hline 1745 & 8 & 1 & 2 & 0 & & 0 \\
\hline 1745 & 9 & 1 & 2 & 0 & & 0 \\
\hline
\end{tabular}


Table III. (continued).

\begin{tabular}{|c|c|c|c|c|c|c|}
\hline year & month & $\begin{array}{c}\text { Etna } \\
\text { eruptions }\end{array}$ & VEI & $\begin{array}{l}\text { Stromboli } \\
\text { eruptions }\end{array}$ & VEI & $\begin{array}{c}\text { Vulcano } \\
\text { eruptions }\end{array}$ \\
\hline 1747 & 9 & 1 & 2 & 0 & & 0 \\
\hline 1747 & 10 & 1 & 2 & 0 & & 0 \\
\hline 1747 & 11 & 1 & 2 & 0 & & 0 \\
\hline 1747 & 12 & 1 & 2 & 0 & & 0 \\
\hline 1748 & 1 & 1 & 2 & 0 & & 0 \\
\hline 1748 & 2 & 1 & 2 & 0 & & 0 \\
\hline 1748 & 3 & 1 & 2 & 0 & & 0 \\
\hline 1748 & 4 & 1 & 2 & 0 & & 0 \\
\hline 1748 & 5 & 1 & 2 & 0 & & 0 \\
\hline 1748 & 6 & 1 & 2 & 0 & & 0 \\
\hline 1748 & 7 & 1 & 2 & 0 & & 0 \\
\hline 1748 & 8 & 1 & 2 & 0 & & 0 \\
\hline 1748 & 9 & 1 & 2 & 0 & & 0 \\
\hline 1748 & 10 & 1 & 2 & 0 & & 0 \\
\hline 1748 & 11 & 1 & 2 & 0 & & 0 \\
\hline 1748 & 12 & 1 & 2 & 0 & & 0 \\
\hline 1749 & 1 & 1 & 2 & 0 & & 0 \\
\hline 1749 & 2 & 1 & 2 & 0 & & 0 \\
\hline 1749 & 3 & 1 & 2 & 0 & & 0 \\
\hline 1752 & 12 & 1 & 2 & 0 & & 0 \\
\hline 1753 & 1 & 1 & 2 & 0 & & 0 \\
\hline 1753 & 2 & 1 & 2 & 0 & & 0 \\
\hline 1753 & 3 & 1 & 2 & 0 & & 0 \\
\hline 1753 & 4 & 1 & 2 & 0 & & 0 \\
\hline 1753 & 5 & 1 & 2 & 0 & & 0 \\
\hline 1753 & 6 & 1 & 2 & 0 & & 0 \\
\hline 1753 & 7 & 1 & 2 & 0 & & 0 \\
\hline 1753 & 8 & 1 & 2 & 0 & & 0 \\
\hline 1753 & 9 & 1 & 2 & 0 & & 0 \\
\hline 1753 & 10 & 1 & 2 & 0 & & 0 \\
\hline 1753 & 11 & 1 & 2 & 0 & & 0 \\
\hline 1753 & 12 & 1 & 2 & 0 & & 0 \\
\hline 1754 & 1 & 1 & 2 & 0 & & 0 \\
\hline 1754 & 2 & 1 & 2 & 0 & & 0 \\
\hline 1754 & 3 & 1 & 2 & 0 & & 0 \\
\hline 1754 & 4 & 1 & 2 & 0 & & 0 \\
\hline 1754 & 5 & 1 & 2 & 0 & & 0 \\
\hline 1754 & 6 & 1 & 2 & 0 & & 0 \\
\hline 1754 & 7 & 1 & 2 & 0 & & 0 \\
\hline 1754 & 8 & 1 & 2 & 0 & & 0 \\
\hline
\end{tabular}


Table III. (continued).

\begin{tabular}{|c|c|c|c|c|c|c|}
\hline year & month & $\begin{array}{c}\text { Etna } \\
\text { eruptions }\end{array}$ & VEI & $\begin{array}{l}\text { Stromboli } \\
\text { eruptions }\end{array}$ & VEI & $\begin{array}{c}\text { Vulcano } \\
\text { eruptions }\end{array}$ \\
\hline 1754 & 9 & 1 & 2 & 0 & & 0 \\
\hline 1754 & 10 & 1 & 2 & 0 & & 0 \\
\hline 1754 & 11 & 1 & 2 & 0 & & 0 \\
\hline 1754 & 12 & 1 & 2 & 0 & & 0 \\
\hline 1755 & 1 & 1 & 2 & 0 & & 0 \\
\hline 1755 & 2 & 1 & 2 & 0 & & 0 \\
\hline 1755 & 3 & 1 & 2 & 0 & & 0 \\
\hline 1755 & 4 & 1 & 2 & 0 & & 0 \\
\hline 1755 & 5 & 1 & 2 & 0 & & 0 \\
\hline 1755 & 6 & 1 & 2 & 0 & & 0 \\
\hline 1755 & 7 & 1 & 2 & 0 & & 0 \\
\hline 1755 & 8 & 1 & 2 & 0 & & 0 \\
\hline 1755 & 9 & 1 & 2 & 0 & & 0 \\
\hline 1755 & 10 & 1 & 2 & 0 & & 0 \\
\hline 1755 & 11 & 1 & 2 & 0 & & 0 \\
\hline 1755 & 12 & 1 & 2 & 0 & & 0 \\
\hline 1756 & 1 & 1 & 2 & 0 & & 0 \\
\hline 1756 & 2 & 1 & 2 & 0 & & 0 \\
\hline 1756 & 3 & 1 & 2 & 0 & & 0 \\
\hline 1756 & 4 & 1 & 2 & 0 & & 0 \\
\hline 1756 & 5 & 1 & 2 & 0 & & 0 \\
\hline 1756 & 6 & 1 & 2 & 0 & & 0 \\
\hline 1756 & 7 & 1 & 2 & 0 & & 0 \\
\hline 1756 & 8 & 1 & 2 & 0 & & 0 \\
\hline 1756 & 9 & 1 & 2 & 0 & & 0 \\
\hline 1756 & 10 & 1 & 2 & 0 & & 0 \\
\hline 1756 & 11 & 1 & 2 & 0 & & 0 \\
\hline 1756 & 12 & 1 & 2 & 0 & & 0 \\
\hline 1757 & 1 & 1 & 2 & 0 & & 0 \\
\hline 1757 & 2 & 1 & 2 & 0 & & 0 \\
\hline 1757 & 3 & 1 & 2 & 0 & & 0 \\
\hline 1757 & 4 & 1 & 2 & 0 & & 0 \\
\hline 1757 & 5 & 1 & 2 & 0 & & 0 \\
\hline 1757 & 6 & 1 & 2 & 0 & & 0 \\
\hline 1757 & 7 & 1 & 2 & 0 & & 0 \\
\hline 1757 & 8 & 1 & 2 & 0 & & 0 \\
\hline 1757 & 9 & 1 & 2 & 0 & & 0 \\
\hline 1757 & 10 & 1 & 2 & 0 & & 0 \\
\hline 1757 & 11 & 1 & 2 & 0 & & 0 \\
\hline 1757 & 12 & 1 & 2 & 0 & & 0 \\
\hline
\end{tabular}


Table III. (continued).

\begin{tabular}{|c|c|c|c|c|c|c|}
\hline year & month & $\begin{array}{c}\text { Etna } \\
\text { eruptions }\end{array}$ & VEI & $\begin{array}{l}\text { Stromboli } \\
\text { eruptions }\end{array}$ & VEI & $\begin{array}{c}\text { Vulcano } \\
\text { eruptions }\end{array}$ \\
\hline 1758 & 1 & 1 & 2 & 0 & & 0 \\
\hline 1758 & 11 & 1 & 2 & 0 & & 0 \\
\hline 1758 & 12 & 1 & 2 & 0 & & 0 \\
\hline 1759 & 1 & 1 & 2 & 0 & & 0 \\
\hline 1759 & 2 & 1 & 2 & 0 & & 0 \\
\hline 1763 & 2 & 1 & 2 & 0 & & 0 \\
\hline 1763 & 3 & 1 & 2 & 0 & & 0 \\
\hline 1763 & 6 & 1 & 3 & 0 & & 0 \\
\hline 1763 & 7 & 1 & 3 & 0 & & 0 \\
\hline 1763 & 8 & 1 & 3 & 0 & & 0 \\
\hline 1763 & 9 & 1 & 3 & 0 & & 0 \\
\hline 1766 & 4 & 1 & 2 & 0 & & 0 \\
\hline 1766 & 5 & 1 & 2 & 0 & & 0 \\
\hline 1766 & 6 & 1 & 2 & 0 & & 0 \\
\hline 1766 & 7 & 1 & 2 & 0 & & 0 \\
\hline 1766 & 8 & 1 & 2 & 0 & & 0 \\
\hline 1766 & 9 & 1 & 2 & 0 & & 0 \\
\hline 1766 & 10 & 1 & 2 & 0 & & 0 \\
\hline 1766 & 11 & 1 & 2 & 0 & & 0 \\
\hline 1771 & 2 & 0 & & 0 & & 1 \\
\hline 1771 & 3 & 0 & & 0 & & 1 \\
\hline 1771 & 4 & 0 & & 0 & & 1 \\
\hline 1771 & 5 & 0 & & 0 & & 1 \\
\hline 1778 & 3 & 0 & & 1 & 2 & 0 \\
\hline 1780 & 4 & 1 & 2 & 0 & & 0 \\
\hline 1780 & 5 & 1 & 2 & 0 & & 0 \\
\hline 1780 & 6 & 1 & 2 & 0 & & 0 \\
\hline 1781 & 3 & 1 & 2 & 0 & & 0 \\
\hline 1781 & 4 & 1 & 2 & 0 & & 0 \\
\hline 1781 & 5 & 1 & 2 & 0 & & 0 \\
\hline 1787 & 6 & 1 & 4 & 0 & & 0 \\
\hline 1787 & 7 & 1 & 4 & 0 & & 0 \\
\hline 1787 & 8 & 1 & 4 & 0 & & 0 \\
\hline 1791 & 2 & 1 & 2 & 0 & & 0 \\
\hline 1791 & 3 & 1 & 2 & 0 & & 0 \\
\hline 1791 & 4 & 1 & 2 & 0 & & 0 \\
\hline 1791 & 5 & 1 & 2 & 0 & & 0 \\
\hline 1791 & 6 & 1 & 2 & 0 & & 0 \\
\hline 1791 & 7 & 1 & 2 & 0 & & 0 \\
\hline 1791 & 8 & 1 & 2 & 0 & & 0 \\
\hline
\end{tabular}


Table III. (continued).

\begin{tabular}{|c|c|c|c|c|c|c|}
\hline year & month & $\begin{array}{c}\text { Etna } \\
\text { eruptions }\end{array}$ & VEI & $\begin{array}{l}\text { Stromboli } \\
\text { eruptions }\end{array}$ & VEI & $\begin{array}{c}\text { Vulcano } \\
\text { eruptions }\end{array}$ \\
\hline 1791 & 9 & 1 & 2 & 0 & & 0 \\
\hline 1792 & 3 & 1 & 2 & 0 & & 0 \\
\hline 1792 & 4 & 1 & 2 & 0 & & 0 \\
\hline 1792 & 5 & 1 & 3 & 0 & & 0 \\
\hline 1792 & 6 & 1 & 3 & 0 & & 0 \\
\hline 1792 & 7 & 1 & 3 & 0 & & 0 \\
\hline 1792 & 8 & 1 & 3 & 0 & & 0 \\
\hline 1792 & 9 & 1 & 3 & 0 & & 0 \\
\hline 1792 & 10 & 1 & 3 & 0 & & 0 \\
\hline 1792 & 11 & 1 & 3 & 0 & & 0 \\
\hline 1792 & 12 & 1 & 3 & 0 & & 0 \\
\hline 1793 & 1 & 1 & 3 & 0 & & 0 \\
\hline 1793 & 2 & 1 & 3 & 0 & & 0 \\
\hline 1793 & 3 & 1 & 3 & 0 & & 0 \\
\hline 1793 & 4 & 1 & 3 & 0 & & 0 \\
\hline 1793 & 5 & 1 & 3 & 0 & & 0 \\
\hline 1797 & 12 & 1 & 2 & 0 & & 0 \\
\hline 1798 & 1 & 1 & 2 & 0 & & 0 \\
\hline 1798 & 2 & 1 & 2 & 0 & & 0 \\
\hline 1798 & 3 & 1 & 2 & 0 & & 0 \\
\hline 1798 & 4 & 1 & 2 & 0 & & 0 \\
\hline 1798 & 5 & 1 & 2 & 0 & & 0 \\
\hline 1798 & 6 & 1 & 2 & 0 & & 0 \\
\hline 1798 & 7 & 1 & 2 & 0 & & 0 \\
\hline 1798 & 8 & 1 & 2 & 0 & & 0 \\
\hline 1798 & 9 & 1 & 2 & 0 & & 0 \\
\hline 1798 & 10 & 1 & 2 & 0 & & 0 \\
\hline 1798 & 11 & 1 & 2 & 0 & & 0 \\
\hline 1798 & 12 & 1 & 2 & 0 & & 0 \\
\hline 1799 & 1 & 1 & 2 & 0 & & 0 \\
\hline 1799 & 2 & 1 & 2 & 0 & & 0 \\
\hline 1799 & 3 & 1 & 2 & 0 & & 0 \\
\hline 1799 & 4 & 1 & 2 & 0 & & 0 \\
\hline 1799 & 5 & 1 & 2 & 0 & & 0 \\
\hline 1799 & 6 & 1 & 2 & 0 & & 0 \\
\hline 1799 & 7 & 1 & 2 & 0 & & 0 \\
\hline 1799 & 8 & 1 & 2 & 0 & & 0 \\
\hline 1799 & 9 & 1 & 2 & 0 & & 0 \\
\hline 1799 & 10 & 1 & 2 & 0 & & 0 \\
\hline 1799 & 11 & 1 & 2 & 0 & & 0 \\
\hline
\end{tabular}


Table III. (continued).

\begin{tabular}{|c|c|c|c|c|c|c|}
\hline year & month & $\begin{array}{c}\text { Etna } \\
\text { eruptions }\end{array}$ & VEI & $\begin{array}{l}\text { Stromboli } \\
\text { eruptions }\end{array}$ & VEI & $\begin{array}{c}\text { Vulcano } \\
\text { eruptions }\end{array}$ \\
\hline 1799 & 12 & 1 & 2 & 0 & & 0 \\
\hline 1800 & 1 & 1 & 2 & 0 & & 0 \\
\hline 1800 & 2 & 1 & 2 & 0 & & 0 \\
\hline 1800 & 3 & 1 & 2 & 0 & & 0 \\
\hline 1800 & 4 & 1 & 2 & 0 & & 0 \\
\hline 1800 & 5 & 1 & 2 & 0 & & 0 \\
\hline 1800 & 6 & 1 & 2 & 0 & & 0 \\
\hline 1800 & 7 & 1 & 2 & 0 & & 0 \\
\hline 1800 & 8 & 1 & 2 & 0 & & 0 \\
\hline 1800 & 9 & 1 & 2 & 0 & & 0 \\
\hline 1800 & 10 & 1 & 2 & 0 & & 0 \\
\hline 1800 & 11 & 1 & 2 & 0 & & 0 \\
\hline 1800 & 12 & 1 & 2 & 0 & & 0 \\
\hline 1801 & 1 & 1 & 2 & 0 & & 0 \\
\hline 1802 & 11 & 1 & 2 & 0 & & 0 \\
\hline 1803 & 12 & 1 & 2 & 0 & & 0 \\
\hline 1804 & 1 & 1 & 2 & 0 & & 0 \\
\hline 1804 & 2 & 1 & 2 & 0 & & 0 \\
\hline 1804 & 3 & 1 & 2 & 0 & & 0 \\
\hline 1804 & 4 & 1 & 2 & 0 & & 0 \\
\hline 1804 & 5 & 1 & 2 & 0 & & 0 \\
\hline 1804 & 6 & 1 & 2 & 0 & & 0 \\
\hline 1804 & 7 & 1 & 2 & 0 & & 0 \\
\hline 1804 & 8 & 1 & 2 & 0 & & 0 \\
\hline 1804 & 9 & 1 & 2 & 0 & & 0 \\
\hline 1804 & 10 & 1 & 2 & 0 & & 0 \\
\hline 1804 & 11 & 1 & 2 & 0 & & 0 \\
\hline 1804 & 12 & 1 & 2 & 0 & & 0 \\
\hline 1805 & 1 & 1 & 2 & 0 & & 0 \\
\hline 1805 & 2 & 1 & 2 & 0 & & 0 \\
\hline 1805 & 3 & 1 & 2 & 0 & & 0 \\
\hline 1805 & 4 & 1 & 2 & 0 & & 0 \\
\hline 1805 & 5 & 1 & 2 & 0 & & 0 \\
\hline 1805 & 6 & 1 & 2 & 0 & & 0 \\
\hline 1805 & 7 & 1 & 2 & 0 & & 0 \\
\hline 1805 & 8 & 1 & 2 & 0 & & 0 \\
\hline 1805 & 9 & 1 & 2 & 0 & & 0 \\
\hline 1805 & 10 & 1 & 2 & 0 & & 0 \\
\hline 1805 & 11 & 1 & 2 & 0 & & 0 \\
\hline 1805 & 12 & 1 & 2 & 0 & & 0 \\
\hline
\end{tabular}


Table III. (continued).

\begin{tabular}{|c|c|c|c|c|c|c|}
\hline year & month & $\begin{array}{c}\text { Etna } \\
\text { eruptions }\end{array}$ & VEI & $\begin{array}{l}\text { Stromboli } \\
\text { eruptions }\end{array}$ & VEI & $\begin{array}{c}\text { Vulcano } \\
\text { eruptions }\end{array}$ \\
\hline 1806 & 1 & 1 & 2 & 0 & & 0 \\
\hline 1806 & 2 & 1 & 2 & 0 & & 0 \\
\hline 1806 & 3 & 1 & 2 & 0 & & 0 \\
\hline 1806 & 4 & 1 & 2 & 0 & & 0 \\
\hline 1806 & 5 & 1 & 2 & 0 & & 0 \\
\hline 1806 & 6 & 1 & 2 & 0 & & 0 \\
\hline 1806 & 7 & 1 & 2 & 0 & & 0 \\
\hline 1806 & 8 & 1 & 2 & 0 & & 0 \\
\hline 1806 & 9 & 1 & 2 & 0 & & 0 \\
\hline 1806 & 10 & 1 & 2 & 0 & & 0 \\
\hline 1806 & 11 & 1 & 2 & 0 & & 0 \\
\hline 1806 & 12 & 1 & 2 & 0 & & 0 \\
\hline 1807 & 1 & 1 & 2 & 0 & & 0 \\
\hline 1807 & 2 & 1 & 2 & 0 & & 0 \\
\hline 1807 & 3 & 1 & 2 & 0 & & 0 \\
\hline 1807 & 4 & 1 & 2 & 0 & & 0 \\
\hline 1807 & 5 & 1 & 2 & 0 & & 0 \\
\hline 1807 & 6 & 1 & 2 & 0 & & 0 \\
\hline 1807 & 7 & 1 & 2 & 0 & & 0 \\
\hline 1807 & 8 & 1 & 2 & 0 & & 0 \\
\hline 1807 & 9 & 1 & 2 & 0 & & 0 \\
\hline 1807 & 10 & 1 & 2 & 0 & & 0 \\
\hline 1807 & 11 & 1 & 2 & 0 & & 0 \\
\hline 1807 & 12 & 1 & 2 & 0 & & 0 \\
\hline 1808 & 1 & 1 & 2 & 0 & & 0 \\
\hline 1808 & 2 & 1 & 2 & 0 & & 0 \\
\hline 1808 & 3 & 1 & 2 & 0 & & 0 \\
\hline 1808 & 4 & 1 & 2 & 0 & & 0 \\
\hline 1808 & 5 & 1 & 2 & 0 & & 0 \\
\hline 1808 & 6 & 1 & 2 & 0 & & 0 \\
\hline 1808 & 7 & 1 & 2 & 0 & & 0 \\
\hline 1808 & 8 & 1 & 2 & 0 & & 0 \\
\hline 1808 & 9 & 1 & 2 & 0 & & 0 \\
\hline 1808 & 10 & 1 & 2 & 0 & & 0 \\
\hline 1808 & 11 & 1 & 2 & 0 & & 0 \\
\hline 1808 & 12 & 1 & 2 & 0 & & 0 \\
\hline 1809 & 1 & 1 & 2 & 0 & & 0 \\
\hline 1809 & 2 & 1 & 2 & 0 & & 0 \\
\hline 1809 & 3 & 1 & 2 & 0 & & 0 \\
\hline 1809 & 4 & 1 & 2 & 0 & & 0 \\
\hline
\end{tabular}


Table III. (continued).

\begin{tabular}{|c|c|c|c|c|c|c|}
\hline year & month & $\begin{array}{c}\text { Etna } \\
\text { eruptions }\end{array}$ & VEI & $\begin{array}{l}\text { Stromboli } \\
\text { eruptions }\end{array}$ & VEI & $\begin{array}{c}\text { Vulcano } \\
\text { eruptions }\end{array}$ \\
\hline 1809 & 5 & 1 & 2 & 0 & & 0 \\
\hline 1809 & 6 & 1 & 2 & 0 & & 0 \\
\hline 1809 & 7 & 1 & 2 & 0 & & 0 \\
\hline 1809 & 8 & 1 & 2 & 0 & & 0 \\
\hline 1809 & 9 & 1 & 2 & 0 & & 0 \\
\hline 1809 & 10 & 1 & 2 & 0 & & 0 \\
\hline 1809 & 11 & 1 & 2 & 0 & & 0 \\
\hline 1811 & 10 & 1 & 2 & 0 & & 0 \\
\hline 1811 & 11 & 1 & 2 & 0 & & 0 \\
\hline 1811 & 12 & 1 & 2 & 0 & & 0 \\
\hline 1812 & 1 & 1 & 2 & 0 & & 0 \\
\hline 1812 & 2 & 1 & 2 & 0 & & 0 \\
\hline 1812 & 3 & 1 & 2 & 0 & & 0 \\
\hline 1812 & 4 & 1 & 2 & 0 & & 0 \\
\hline 1812 & 5 & 1 & 2 & 0 & & 0 \\
\hline 1819 & 5 & 1 & 3 & 0 & & 0 \\
\hline 1819 & 6 & 1 & 3 & 0 & & 0 \\
\hline 1819 & 7 & 1 & 3 & 0 & & 0 \\
\hline 1819 & 8 & 1 & 3 & 0 & & 0 \\
\hline 1822 & 10 & 0 & & 1 & 3 & 0 \\
\hline 1828 & 8 & 1 & 2 & 0 & & 0 \\
\hline 1828 & 9 & 1 & 2 & 0 & & 0 \\
\hline 1828 & 10 & 1 & 2 & 0 & & 0 \\
\hline 1828 & 11 & 1 & 2 & 0 & & 0 \\
\hline 1828 & 12 & 1 & 2 & 0 & & 0 \\
\hline 1829 & 1 & 1 & 2 & 0 & & 0 \\
\hline 1829 & 2 & 1 & 2 & 0 & & 0 \\
\hline 1829 & 3 & 1 & 2 & 0 & & 0 \\
\hline 1829 & 4 & 1 & 2 & 0 & & 0 \\
\hline 1829 & 5 & 1 & 2 & 0 & & 0 \\
\hline 1829 & 6 & 1 & 2 & 0 & & 0 \\
\hline 1829 & 7 & 1 & 2 & 0 & & 0 \\
\hline 1829 & 8 & 1 & 2 & 0 & & 0 \\
\hline 1829 & 9 & 1 & 2 & 0 & & 0 \\
\hline 1829 & 10 & 1 & 2 & 0 & & 0 \\
\hline 1829 & 11 & 1 & 2 & 0 & & 0 \\
\hline 1829 & 12 & 1 & 2 & 0 & & 0 \\
\hline 1830 & 1 & 1 & 2 & 0 & & 0 \\
\hline 1830 & 2 & 1 & 2 & 0 & & 0 \\
\hline 1830 & 3 & 1 & 2 & 0 & & 0 \\
\hline
\end{tabular}


Table III. (continued).

\begin{tabular}{|c|c|c|c|c|c|c|}
\hline year & month & $\begin{array}{c}\text { Etna } \\
\text { eruptions }\end{array}$ & VEI & $\begin{array}{l}\text { Stromboli } \\
\text { eruptions }\end{array}$ & VEI & $\begin{array}{c}\text { Vulcano } \\
\text { eruptions }\end{array}$ \\
\hline 1830 & 4 & 1 & 2 & 0 & & 0 \\
\hline 1830 & 5 & 1 & 2 & 0 & & 0 \\
\hline 1830 & 6 & 1 & 2 & 0 & & 0 \\
\hline 1830 & 7 & 1 & 2 & 0 & & 0 \\
\hline 1830 & 8 & 1 & 2 & 0 & & 0 \\
\hline 1830 & 9 & 1 & 2 & 0 & & 0 \\
\hline 1830 & 10 & 1 & 2 & 0 & & 0 \\
\hline 1830 & 11 & 1 & 2 & 0 & & 0 \\
\hline 1830 & 12 & 1 & 2 & 0 & & 0 \\
\hline 1831 & 1 & 1 & 2 & 0 & & 0 \\
\hline 1831 & 2 & 1 & 2 & 0 & & 0 \\
\hline 1831 & 3 & 1 & 2 & 0 & & 0 \\
\hline 1831 & 4 & 1 & 2 & 0 & & 0 \\
\hline 1831 & 5 & 1 & 2 & 0 & & 0 \\
\hline 1831 & 6 & 1 & 2 & 0 & & 0 \\
\hline 1831 & 7 & 1 & 2 & 0 & & 0 \\
\hline 1831 & 8 & 1 & 2 & 0 & & 0 \\
\hline 1831 & 9 & 1 & 2 & 0 & & 0 \\
\hline 1831 & 10 & 1 & 2 & 0 & & 0 \\
\hline 1831 & 11 & 1 & 2 & 0 & & 0 \\
\hline 1831 & 12 & 1 & 2 & 0 & & 0 \\
\hline 1832 & 1 & 1 & 2 & 0 & & 0 \\
\hline 1832 & 2 & 1 & 2 & 0 & & 0 \\
\hline 1832 & 3 & 1 & 2 & 0 & & 0 \\
\hline 1832 & 4 & 1 & 2 & 0 & & 0 \\
\hline 1832 & 5 & 1 & 2 & 0 & & 0 \\
\hline 1832 & 6 & 1 & 2 & 0 & & 0 \\
\hline 1832 & 7 & 1 & 2 & 0 & & 0 \\
\hline 1832 & 8 & 1 & 2 & 0 & & 0 \\
\hline 1832 & 9 & 1 & 2 & 0 & & 0 \\
\hline 1832 & 10 & 1 & 2 & 0 & & 0 \\
\hline 1832 & 11 & 1 & 2 & 0 & & 0 \\
\hline 1833 & 3 & 1 & 2 & 0 & & 0 \\
\hline 1838 & 7 & 1 & 2 & 0 & & 0 \\
\hline 1838 & 8 & 1 & 2 & 0 & & 0 \\
\hline 1838 & 9 & 1 & 2 & 0 & & 0 \\
\hline 1838 & 10 & 1 & 2 & 0 & & 0 \\
\hline 1838 & 11 & 1 & 2 & 0 & & 0 \\
\hline 1838 & 12 & 1 & 2 & 0 & & 0 \\
\hline 1839 & 1 & 1 & 2 & 0 & & 0 \\
\hline
\end{tabular}


Table III. (continued).

\begin{tabular}{|c|c|c|c|c|c|c|}
\hline year & month & $\begin{array}{c}\text { Etna } \\
\text { eruptions }\end{array}$ & VEI & $\begin{array}{l}\text { Stromboli } \\
\text { eruptions }\end{array}$ & VEI & $\begin{array}{c}\text { Vulcano } \\
\text { eruptions }\end{array}$ \\
\hline 1839 & 2 & 1 & 2 & 0 & & 0 \\
\hline 1842 & 11 & 1 & 2 & 0 & & 0 \\
\hline 1842 & 12 & 1 & 2 & 0 & & 0 \\
\hline 1843 & 11 & 1 & 2 & 0 & & 0 \\
\hline 1852 & 8 & 1 & 2 & 0 & & 0 \\
\hline 1852 & 9 & 1 & 2 & 0 & & 0 \\
\hline 1852 & 10 & 1 & 2 & 0 & & 0 \\
\hline 1852 & 11 & 1 & 2 & 0 & & 0 \\
\hline 1852 & 12 & 1 & 2 & 0 & & 0 \\
\hline 1853 & 1 & 1 & 2 & 0 & & 0 \\
\hline 1853 & 2 & 1 & 2 & 0 & & 0 \\
\hline 1853 & 3 & 1 & 2 & 0 & & 0 \\
\hline 1853 & 4 & 1 & 2 & 0 & & 0 \\
\hline 1853 & 5 & 1 & 2 & 0 & & 0 \\
\hline 1855 & 10 & 0 & & 1 & 2 & 0 \\
\hline 1856 & 7 & 0 & & 1 & 2 & 0 \\
\hline 1863 & 5 & 1 & 2 & 0 & & 0 \\
\hline 1863 & 6 & 1 & 2 & 0 & & 0 \\
\hline 1863 & 7 & 1 & 2 & 0 & & 0 \\
\hline 1864 & 8 & 1 & 2 & 0 & & 0 \\
\hline 1864 & 9 & 1 & 2 & 0 & & 0 \\
\hline 1865 & 1 & 1 & 2 & 1 & 2 & 0 \\
\hline 1865 & 2 & 1 & 2 & 1 & 2 & 0 \\
\hline 1865 & 3 & 1 & 2 & 0 & & 0 \\
\hline 1865 & 4 & 1 & 2 & 0 & & 0 \\
\hline 1865 & 5 & 1 & 2 & 0 & & 0 \\
\hline 1865 & 6 & 1 & 2 & 0 & & 0 \\
\hline 1868 & 11 & 1 & 3 & 0 & & 0 \\
\hline 1868 & 12 & 1 & 3 & 0 & & 0 \\
\hline 1873 & 9 & 0 & & 0 & & 1 \\
\hline 1873 & 10 & 0 & & 0 & & 1 \\
\hline 1873 & 11 & 0 & & 0 & & 1 \\
\hline 1873 & 12 & 0 & & 0 & & 1 \\
\hline 1874 & 1 & 0 & & 0 & & 1 \\
\hline 1874 & 2 & 0 & & 0 & & 1 \\
\hline 1874 & 3 & 0 & & 0 & & 1 \\
\hline 1874 & 4 & 0 & & 0 & & 1 \\
\hline 1874 & 5 & 1 & 2 & 0 & & 1 \\
\hline 1874 & 6 & 1 & 2 & 1 & 2 & 1 \\
\hline 1874 & 7 & 1 & 2 & 0 & & 1 \\
\hline
\end{tabular}


Table III. (continued).

\begin{tabular}{|c|c|c|c|c|c|c|}
\hline year & month & $\begin{array}{c}\text { Etna } \\
\text { eruptions }\end{array}$ & VEI & $\begin{array}{l}\text { Stromboli } \\
\text { eruptions }\end{array}$ & VEI & $\begin{array}{c}\text { Vulcano } \\
\text { eruptions }\end{array}$ \\
\hline 1874 & 8 & 1 & 2 & 0 & & 1 \\
\hline 1874 & 9 & 0 & & 0 & & 1 \\
\hline 1874 & 10 & 0 & & 0 & & 1 \\
\hline 1874 & 11 & 0 & & 0 & & 1 \\
\hline 1874 & 12 & 1 & 2 & 0 & & 1 \\
\hline 1875 & 1 & 1 & 2 & 0 & & 1 \\
\hline 1875 & 2 & 1 & 2 & 0 & & 1 \\
\hline 1875 & 3 & 1 & 2 & 0 & & 1 \\
\hline 1875 & 4 & 1 & 2 & 0 & & 1 \\
\hline 1875 & 5 & 1 & 2 & 0 & & 1 \\
\hline 1875 & 6 & 1 & 2 & 0 & & 1 \\
\hline 1875 & 7 & 1 & 2 & 0 & & 1 \\
\hline 1875 & 8 & 1 & 2 & 0 & & 1 \\
\hline 1875 & 9 & 1 & 2 & 0 & & 1 \\
\hline 1875 & 10 & 1 & 2 & 0 & & 1 \\
\hline 1875 & 11 & 1 & 2 & 0 & & 1 \\
\hline 1875 & 12 & 1 & 2 & 0 & & 1 \\
\hline 1876 & 1 & 1 & 2 & 0 & & 1 \\
\hline 1876 & 2 & 1 & 2 & 0 & & 1 \\
\hline 1876 & 3 & 1 & 2 & 0 & & 1 \\
\hline 1876 & 4 & 1 & 2 & 0 & & 1 \\
\hline 1876 & 5 & 1 & 2 & 0 & & 1 \\
\hline 1876 & 6 & 1 & 2 & 0 & & 1 \\
\hline 1876 & 7 & 1 & 2 & 0 & & 1 \\
\hline 1876 & 8 & 1 & 2 & 0 & & 1 \\
\hline 1876 & 9 & 1 & 2 & 0 & & 1 \\
\hline 1876 & 10 & 1 & 2 & 0 & & 1 \\
\hline 1876 & 11 & 1 & 2 & 0 & & 1 \\
\hline 1876 & 12 & 1 & 2 & 0 & & 1 \\
\hline 1877 & 1 & 1 & 2 & 0 & & 1 \\
\hline 1877 & 2 & 1 & 2 & 0 & & 1 \\
\hline 1877 & 3 & 1 & 2 & 0 & & 1 \\
\hline 1877 & 4 & 1 & 2 & 0 & & 1 \\
\hline 1877 & 5 & 1 & 2 & 0 & & 1 \\
\hline 1877 & 6 & 1 & 2 & 0 & & 1 \\
\hline 1877 & 7 & 1 & 2 & 0 & & 1 \\
\hline 1877 & 8 & 1 & 2 & 0 & & 1 \\
\hline 1877 & 9 & 1 & 2 & 0 & & 1 \\
\hline 1877 & 10 & 1 & 2 & 0 & & 1 \\
\hline 1877 & 11 & 1 & 2 & 0 & & 1 \\
\hline
\end{tabular}


Table III. (continued).

\begin{tabular}{|c|c|c|c|c|c|c|}
\hline year & month & $\begin{array}{c}\text { Etna } \\
\text { eruptions }\end{array}$ & VEI & $\begin{array}{l}\text { Stromboli } \\
\text { eruptions }\end{array}$ & VEI & $\begin{array}{c}\text { Vulcano } \\
\text { eruptions }\end{array}$ \\
\hline 1877 & 12 & 1 & 2 & 0 & & 1 \\
\hline 1878 & 1 & 1 & 2 & 0 & & 1 \\
\hline 1878 & 2 & 1 & 2 & 0 & & 1 \\
\hline 1878 & 3 & 1 & 2 & 0 & & 1 \\
\hline 1878 & 4 & 1 & 2 & 0 & & 1 \\
\hline 1878 & 5 & 1 & 2 & 0 & & 1 \\
\hline 1878 & 6 & 1 & 2 & 0 & & 1 \\
\hline 1878 & 7 & 1 & 2 & 0 & & 1 \\
\hline 1878 & 8 & 1 & 2 & 0 & & 1 \\
\hline 1878 & 9 & 1 & 2 & 0 & & 1 \\
\hline 1878 & 10 & 1 & 2 & 0 & & 1 \\
\hline 1878 & 11 & 1 & 2 & 0 & & 1 \\
\hline 1878 & 12 & 1 & 2 & 0 & & 1 \\
\hline 1879 & 1 & 1 & 2 & 0 & & 1 \\
\hline 1879 & 2 & 1 & 2 & 1 & 2 & 0 \\
\hline 1879 & 3 & 1 & 2 & 0 & & 0 \\
\hline 1879 & 4 & 1 & 2 & 0 & & 0 \\
\hline 1879 & 5 & 1 & 3 & 0 & & 0 \\
\hline 1879 & 6 & 1 & 3 & 1 & 2 & 0 \\
\hline 1879 & 7 & 1 & 2 & 0 & & 0 \\
\hline 1879 & 8 & 1 & 2 & 0 & & 0 \\
\hline 1879 & 9 & 1 & 2 & 0 & & 0 \\
\hline 1879 & 10 & 1 & 2 & 0 & & 0 \\
\hline 1879 & 11 & 1 & 2 & 0 & & 0 \\
\hline 1879 & 12 & 1 & 2 & 0 & & 0 \\
\hline 1880 & 1 & 1 & 2 & 0 & & 0 \\
\hline 1880 & 2 & 1 & 2 & 0 & & 0 \\
\hline 1880 & 3 & 1 & 2 & 0 & & 0 \\
\hline 1880 & 4 & 1 & 2 & 0 & & 0 \\
\hline 1880 & 5 & 1 & 2 & 0 & & 0 \\
\hline 1880 & 6 & 1 & 2 & 0 & & 0 \\
\hline 1880 & 7 & 1 & 2 & 0 & & 0 \\
\hline 1880 & 8 & 1 & 2 & 0 & & 0 \\
\hline 1880 & 9 & 1 & 2 & 0 & & 0 \\
\hline 1880 & 10 & 1 & 2 & 0 & & 0 \\
\hline 1880 & 11 & 1 & 2 & 0 & & 0 \\
\hline 1880 & 12 & 1 & 2 & 0 & & 0 \\
\hline 1881 & 1 & 1 & 2 & 0 & & 0 \\
\hline 1881 & 2 & 1 & 2 & 0 & & 0 \\
\hline 1881 & 3 & 1 & 2 & 0 & & 0 \\
\hline
\end{tabular}


Table III. (continued).

\begin{tabular}{|c|c|c|c|c|c|c|}
\hline year & month & $\begin{array}{c}\text { Etna } \\
\text { eruptions }\end{array}$ & VEI & $\begin{array}{l}\text { Stromboli } \\
\text { eruptions }\end{array}$ & VEI & $\begin{array}{c}\text { Vulcano } \\
\text { eruptions }\end{array}$ \\
\hline 1881 & 4 & 1 & 2 & 0 & & 0 \\
\hline 1881 & 5 & 1 & 2 & 0 & & 0 \\
\hline 1881 & 6 & 1 & 2 & 0 & & 0 \\
\hline 1881 & 7 & 1 & 2 & 0 & & 0 \\
\hline 1881 & 8 & 1 & 2 & 0 & & 0 \\
\hline 1881 & 9 & 1 & 2 & 0 & & 0 \\
\hline 1881 & 10 & 1 & 2 & 1 & 2 & 0 \\
\hline 1881 & 11 & 1 & 2 & 0 & & 0 \\
\hline 1881 & 12 & 1 & 2 & 0 & & 0 \\
\hline 1882 & 1 & 1 & 2 & 0 & & 0 \\
\hline 1882 & 2 & 1 & 2 & 0 & & 0 \\
\hline 1882 & 3 & 1 & 2 & 0 & & 0 \\
\hline 1882 & 4 & 1 & 2 & 0 & & 0 \\
\hline 1882 & 5 & 1 & 2 & 0 & & 0 \\
\hline 1882 & 6 & 1 & 2 & 0 & & 0 \\
\hline 1882 & 7 & 1 & 2 & 0 & & 0 \\
\hline 1882 & 8 & 1 & 2 & 0 & & 0 \\
\hline 1882 & 9 & 1 & 2 & 0 & & 0 \\
\hline 1882 & 10 & 1 & 2 & 0 & & 0 \\
\hline 1882 & 11 & 1 & 2 & 1 & 3 & 0 \\
\hline 1882 & 12 & 1 & 2 & 0 & & 0 \\
\hline 1883 & 1 & 1 & 2 & 0 & & 0 \\
\hline 1883 & 2 & 1 & 2 & 0 & & 0 \\
\hline 1883 & 3 & 1 & 2 & 0 & & 0 \\
\hline 1885 & 3 & 0 & & 1 & 3 & 0 \\
\hline 1886 & 1 & 0 & & 0 & & 1 \\
\hline 1886 & 5 & 1 & 3 & 0 & & 0 \\
\hline 1886 & 6 & 1 & 3 & 0 & & 0 \\
\hline 1888 & 8 & 0 & & 0 & & 1 \\
\hline 1888 & 9 & 0 & & 0 & & 1 \\
\hline 1888 & 10 & 0 & & 1 & 2 & 1 \\
\hline 1888 & 11 & 0 & & 1 & 2 & 1 \\
\hline 1888 & 12 & 0 & & 1 & 2 & 1 \\
\hline 1889 & 1 & 0 & & 1 & 2 & 1 \\
\hline 1889 & 2 & 0 & & 1 & 2 & 1 \\
\hline 1889 & 3 & 0 & & 1 & 2 & 1 \\
\hline 1889 & 4 & 0 & & 1 & 2 & 1 \\
\hline 1889 & 5 & 0 & & 1 & 2 & 1 \\
\hline 1889 & 6 & 0 & & 1 & 2 & 1 \\
\hline 1889 & 7 & 0 & & 0 & & 1 \\
\hline
\end{tabular}


Table III. (continued).

\begin{tabular}{|c|c|c|c|c|c|c|}
\hline year & month & $\begin{array}{c}\text { Etna } \\
\text { eruptions }\end{array}$ & VEI & $\begin{array}{l}\text { Stromboli } \\
\text { eruptions }\end{array}$ & VEI & $\begin{array}{c}\text { Vulcano } \\
\text { eruptions }\end{array}$ \\
\hline 1889 & 8 & 0 & & 0 & & 1 \\
\hline 1889 & 9 & 0 & & 0 & & 1 \\
\hline 1889 & 10 & 0 & & 0 & & 1 \\
\hline 1889 & 11 & 0 & & 0 & & 1 \\
\hline 1889 & 12 & 0 & & 0 & & 1 \\
\hline 1890 & 1 & 0 & & 0 & & 1 \\
\hline 1890 & 2 & 0 & & 0 & & 1 \\
\hline 1890 & 3 & 0 & & 0 & & 1 \\
\hline 1891 & 6 & 0 & & 1 & 3 & 0 \\
\hline 1891 & 7 & 0 & & 1 & 3 & 0 \\
\hline 1891 & 8 & 0 & & 1 & 3 & 0 \\
\hline 1892 & 7 & 1 & 2 & 0 & & 0 \\
\hline 1892 & 8 & 1 & 2 & 0 & & 0 \\
\hline 1892 & 9 & 1 & 2 & 0 & & 0 \\
\hline 1892 & 10 & 1 & 2 & 0 & & 0 \\
\hline 1892 & 11 & 1 & 2 & 1 & 2 & 0 \\
\hline 1892 & 12 & 1 & 2 & 0 & & 0 \\
\hline 1893 & 1 & 0 & & 1 & 2 & 0 \\
\hline 1893 & 4 & 1 & 2 & 0 & & 0 \\
\hline 1893 & 5 & 1 & 2 & 0 & & 0 \\
\hline 1893 & 6 & 1 & 2 & 0 & & 0 \\
\hline 1893 & 7 & 1 & 2 & 0 & & 0 \\
\hline 1893 & 8 & 1 & 2 & 0 & & 0 \\
\hline 1893 & 9 & 1 & 2 & 0 & & 0 \\
\hline 1893 & 10 & 1 & 2 & 0 & & 0 \\
\hline 1893 & 11 & 1 & 2 & 1 & 2 & 0 \\
\hline 1893 & 12 & 1 & 2 & 0 & & 0 \\
\hline 1894 & 1 & 1 & 2 & 0 & & 0 \\
\hline 1894 & 2 & 1 & 2 & 0 & & 0 \\
\hline 1894 & 3 & 1 & 2 & 0 & & 0 \\
\hline 1894 & 4 & 1 & 2 & 0 & & 0 \\
\hline 1894 & 5 & 1 & 2 & 0 & & 0 \\
\hline 1894 & 6 & 1 & 2 & 0 & & 0 \\
\hline 1894 & 7 & 1 & 2 & 0 & & 0 \\
\hline 1894 & 8 & 1 & 2 & 0 & & 0 \\
\hline 1894 & 9 & 1 & 2 & 0 & & 0 \\
\hline 1894 & 10 & 1 & 2 & 0 & & 0 \\
\hline 1894 & 11 & 1 & 2 & 0 & & 0 \\
\hline 1894 & 12 & 1 & 2 & 0 & & 0 \\
\hline 1895 & 1 & 1 & 2 & 0 & & 0 \\
\hline
\end{tabular}


Table III. (continued).

\begin{tabular}{|c|c|c|c|c|c|c|}
\hline year & month & $\begin{array}{c}\text { Etna } \\
\text { eruptions }\end{array}$ & VEI & $\begin{array}{l}\text { Stromboli } \\
\text { eruptions }\end{array}$ & VEI & $\begin{array}{c}\text { Vulcano } \\
\text { eruptions }\end{array}$ \\
\hline 1895 & 2 & 1 & 2 & 0 & & 0 \\
\hline 1895 & 3 & 1 & 2 & 1 & 2 & 0 \\
\hline 1895 & 4 & 1 & 2 & 0 & & 0 \\
\hline 1895 & 5 & 1 & 2 & 0 & & 0 \\
\hline 1895 & 6 & 1 & 2 & 0 & & 0 \\
\hline 1895 & 7 & 1 & 2 & 0 & & 0 \\
\hline 1895 & 8 & 1 & 2 & 0 & & 0 \\
\hline 1895 & 9 & 1 & 2 & 0 & & 0 \\
\hline 1895 & 10 & 1 & 2 & 0 & & 0 \\
\hline 1895 & 11 & 1 & 2 & 0 & & 0 \\
\hline 1895 & 12 & 1 & 2 & 0 & & 0 \\
\hline 1896 & 1 & 1 & 2 & 0 & & 0 \\
\hline 1896 & 2 & 1 & 2 & 0 & & 0 \\
\hline 1896 & 3 & 1 & 2 & 0 & & 0 \\
\hline 1896 & 4 & 1 & 2 & 0 & & 0 \\
\hline 1896 & 5 & 1 & 2 & 0 & & 0 \\
\hline 1896 & 6 & 1 & 2 & 0 & & 0 \\
\hline 1896 & 7 & 1 & 2 & 1 & 2 & 0 \\
\hline 1896 & 8 & 1 & 2 & 0 & & 0 \\
\hline 1896 & 9 & 1 & 2 & 0 & & 0 \\
\hline 1896 & 10 & 1 & 2 & 0 & & 0 \\
\hline 1896 & 11 & 1 & 2 & 0 & & 0 \\
\hline 1896 & 12 & 1 & 2 & 0 & & 0 \\
\hline 1897 & 1 & 1 & 2 & 0 & & 0 \\
\hline 1897 & 2 & 1 & 2 & 0 & & 0 \\
\hline 1897 & 3 & 1 & 2 & 0 & & 0 \\
\hline 1897 & 4 & 1 & 2 & 0 & & 0 \\
\hline 1897 & 5 & 1 & 2 & 0 & & 0 \\
\hline 1897 & 6 & 1 & 2 & 0 & & 0 \\
\hline 1897 & 7 & 1 & 2 & 1 & 2 & 0 \\
\hline 1897 & 8 & 1 & 2 & 0 & & 0 \\
\hline 1897 & 9 & 1 & 2 & 0 & & 0 \\
\hline 1897 & 10 & 1 & 2 & 0 & & 0 \\
\hline 1897 & 11 & 1 & 2 & 0 & & 0 \\
\hline 1897 & 12 & 1 & 2 & 0 & & 0 \\
\hline 1898 & 1 & 1 & 2 & 0 & & 0 \\
\hline 1898 & 2 & 1 & 2 & 0 & & 0 \\
\hline 1898 & 3 & 1 & 2 & 0 & & 0 \\
\hline 1898 & 4 & 1 & 2 & 0 & & 0 \\
\hline 1898 & 5 & 1 & 2 & 0 & & 0 \\
\hline
\end{tabular}


Table III. (continued).

\begin{tabular}{|c|c|c|c|c|c|c|}
\hline year & month & $\begin{array}{c}\text { Etna } \\
\text { eruptions }\end{array}$ & VEI & $\begin{array}{l}\text { Stromboli } \\
\text { eruptions }\end{array}$ & VEI & $\begin{array}{c}\text { Vulcano } \\
\text { eruptions }\end{array}$ \\
\hline 1898 & 6 & 1 & 2 & 0 & & 0 \\
\hline 1898 & 8 & 0 & & 1 & 2 & 0 \\
\hline 1899 & 7 & 1 & 3 & 0 & & 0 \\
\hline 1899 & 8 & 1 & 3 & 0 & & 0 \\
\hline 1900 & 8 & 0 & & 1 & 2 & 0 \\
\hline 1900 & 10 & 0 & & 1 & 2 & 0 \\
\hline 1903 & 1 & 0 & & 1 & 2 & 0 \\
\hline 1903 & 2 & 0 & & 1 & 2 & 0 \\
\hline 1903 & 3 & 0 & & 1 & 2 & 0 \\
\hline 1903 & 4 & 0 & & 1 & 2 & 0 \\
\hline 1903 & 5 & 0 & & 1 & 2 & 0 \\
\hline 1903 & 6 & 0 & & 1 & 2 & 0 \\
\hline 1903 & 11 & 0 & & 1 & 2 & 0 \\
\hline 1905 & 4 & 0 & & 1 & 2 & 0 \\
\hline 1906 & 4 & 0 & & 1 & 2 & 0 \\
\hline 1906 & 7 & 0 & & 1 & 2 & 0 \\
\hline 1907 & 1 & 0 & & 1 & 3 & 0 \\
\hline 1907 & 2 & 0 & & 1 & 3 & 0 \\
\hline 1907 & 3 & 0 & & 1 & 3 & 0 \\
\hline 1907 & 4 & 0 & & 1 & 3 & 0 \\
\hline 1907 & 5 & 0 & & 1 & 3 & 0 \\
\hline 1908 & 4 & 1 & 2 & 0 & & 0 \\
\hline 1910 & 3 & 1 & 2 & 0 & & 0 \\
\hline 1910 & 4 & 1 & 2 & 0 & & 0 \\
\hline 1910 & 12 & 1 & 2 & 0 & & 0 \\
\hline 1911 & 1 & 1 & 2 & 0 & & 0 \\
\hline 1911 & 2 & 1 & 2 & 0 & & 0 \\
\hline 1912 & 7 & 0 & & 1 & 2 & 0 \\
\hline 1912 & 8 & 1 & 3 & 1 & 2 & 0 \\
\hline 1913 & 11 & 1 & 2 & 0 & & 0 \\
\hline 1913 & 12 & 1 & 2 & 0 & & 0 \\
\hline 1914 & 1 & 1 & 2 & 0 & & 0 \\
\hline 1914 & 2 & 1 & 2 & 0 & & 0 \\
\hline 1914 & 3 & 1 & 2 & 0 & & 0 \\
\hline 1914 & 4 & 1 & 2 & 0 & & 0 \\
\hline 1914 & 5 & 1 & 2 & 0 & & 0 \\
\hline 1914 & 6 & 1 & 2 & 0 & & 0 \\
\hline 1914 & 7 & 1 & 2 & 0 & & 0 \\
\hline 1914 & 8 & 1 & 2 & 0 & & 0 \\
\hline 1914 & 9 & 1 & 2 & 0 & & 0 \\
\hline
\end{tabular}


Table III. (continued).

\begin{tabular}{|c|c|c|c|c|c|c|}
\hline year & month & $\begin{array}{c}\text { Etna } \\
\text { eruptions }\end{array}$ & VEI & $\begin{array}{l}\text { Stromboli } \\
\text { eruptions }\end{array}$ & VEI & $\begin{array}{c}\text { Vulcano } \\
\text { eruptions }\end{array}$ \\
\hline 1914 & 10 & 1 & 2 & 0 & & 0 \\
\hline 1914 & 11 & 1 & 2 & 0 & & 0 \\
\hline 1914 & 12 & 1 & 2 & 0 & & 0 \\
\hline 1915 & 1 & 1 & 2 & 0 & & 0 \\
\hline 1915 & 2 & 1 & 2 & 0 & & 0 \\
\hline 1915 & 3 & 1 & 2 & 0 & & 0 \\
\hline 1915 & 4 & 1 & 2 & 0 & & 0 \\
\hline 1915 & 5 & 1 & 2 & 0 & & 0 \\
\hline 1915 & 6 & 1 & 2 & 1 & 2 & 0 \\
\hline 1915 & 7 & 1 & 2 & 1 & 2 & 0 \\
\hline 1915 & 8 & 1 & 2 & 1 & 2 & 0 \\
\hline 1915 & 9 & 1 & 2 & 1 & 2 & 0 \\
\hline 1915 & 10 & 1 & 2 & 1 & 2 & 0 \\
\hline 1915 & 11 & 1 & 2 & 1 & 2 & 0 \\
\hline 1915 & 12 & 1 & 2 & 0 & & 0 \\
\hline 1916 & 1 & 1 & 2 & 0 & & 0 \\
\hline 1916 & 2 & 1 & 2 & 0 & & 0 \\
\hline 1916 & 3 & 1 & 2 & 0 & & 0 \\
\hline 1916 & 4 & 1 & 2 & 0 & & 0 \\
\hline 1916 & 5 & 1 & 2 & 0 & & 0 \\
\hline 1916 & 6 & 1 & 2 & 1 & 2 & 0 \\
\hline 1916 & 7 & 1 & 2 & 1 & 2 & 0 \\
\hline 1916 & 8 & 1 & 2 & 0 & & 0 \\
\hline 1916 & 9 & 1 & 2 & 0 & & 0 \\
\hline 1916 & 10 & 1 & 2 & 0 & & 0 \\
\hline 1916 & 11 & 1 & 2 & 0 & & 0 \\
\hline 1916 & 12 & 1 & 2 & 0 & & 0 \\
\hline 1917 & 1 & 1 & 2 & 0 & & 0 \\
\hline 1917 & 2 & 1 & 2 & 0 & & 0 \\
\hline 1917 & 3 & 1 & 2 & 0 & & 0 \\
\hline 1917 & 6 & 1 & 2 & 0 & & 0 \\
\hline 1917 & 7 & 1 & 2 & 0 & & 0 \\
\hline 1919 & 3 & 1 & 2 & 0 & & 0 \\
\hline 1919 & 4 & 1 & 2 & 0 & & 0 \\
\hline 1919 & 5 & 1 & 2 & 1 & 3 & 0 \\
\hline 1919 & 6 & 1 & 2 & 0 & & 0 \\
\hline 1919 & 7 & 1 & 2 & 0 & & 0 \\
\hline 1919 & 8 & 1 & 2 & 0 & & 0 \\
\hline 1919 & 9 & 1 & 2 & 0 & & 0 \\
\hline 1919 & 10 & 1 & 2 & 0 & & 0 \\
\hline
\end{tabular}


Table III. (continued).

\begin{tabular}{|c|c|c|c|c|c|c|}
\hline year & month & $\begin{array}{c}\text { Etna } \\
\text { eruptions }\end{array}$ & VEI & $\begin{array}{l}\text { Stromboli } \\
\text { eruptions }\end{array}$ & VEI & $\begin{array}{c}\text { Vulcano } \\
\text { eruptions }\end{array}$ \\
\hline 1919 & 11 & 1 & 2 & 0 & & 0 \\
\hline 1919 & 12 & 1 & 2 & 0 & & 0 \\
\hline 1920 & 1 & 1 & 2 & 0 & & 0 \\
\hline 1920 & 2 & 1 & 2 & 0 & & 0 \\
\hline 1920 & 3 & 1 & 2 & 0 & & 0 \\
\hline 1920 & 4 & 1 & 2 & 0 & & 0 \\
\hline 1920 & 5 & 1 & 2 & 0 & & 0 \\
\hline 1920 & 6 & 1 & 2 & 0 & & 0 \\
\hline 1920 & 7 & 1 & 2 & 0 & & 0 \\
\hline 1920 & 8 & 1 & 2 & 0 & & 0 \\
\hline 1920 & 9 & 1 & 2 & 0 & & 0 \\
\hline 1920 & 10 & 1 & 2 & 0 & & 0 \\
\hline 1920 & 11 & 1 & 2 & 0 & & 0 \\
\hline 1920 & 12 & 1 & 2 & 0 & & 0 \\
\hline 1921 & 1 & 1 & 2 & 0 & & 0 \\
\hline 1921 & 2 & 1 & 2 & 0 & & 0 \\
\hline 1921 & 3 & 1 & 2 & 0 & & 0 \\
\hline 1921 & 4 & 1 & 2 & 0 & & 0 \\
\hline 1921 & 5 & 1 & 2 & 0 & & 0 \\
\hline 1921 & 6 & 1 & 2 & 1 & 2 & 0 \\
\hline 1921 & 7 & 1 & 2 & 0 & & 0 \\
\hline 1921 & 8 & 1 & 2 & 0 & & 0 \\
\hline 1921 & 9 & 1 & 2 & 0 & & 0 \\
\hline 1921 & 10 & 1 & 2 & 0 & & 0 \\
\hline 1921 & 11 & 1 & 2 & 0 & & 0 \\
\hline 1921 & 12 & 1 & 2 & 0 & & 0 \\
\hline 1922 & 1 & 1 & 2 & 0 & & 0 \\
\hline 1922 & 2 & 1 & 2 & 0 & & 0 \\
\hline 1922 & 3 & 1 & 2 & 0 & & 0 \\
\hline 1922 & 4 & 1 & 2 & 0 & & 0 \\
\hline 1922 & 5 & 1 & 2 & 0 & & 0 \\
\hline 1922 & 6 & 1 & 2 & 0 & & 0 \\
\hline 1922 & 7 & 1 & 2 & 0 & & 0 \\
\hline 1922 & 8 & 1 & 2 & 0 & & 0 \\
\hline 1922 & 9 & 1 & 2 & 0 & & 0 \\
\hline 1922 & 10 & 1 & 2 & 0 & & 0 \\
\hline 1922 & 11 & 1 & 2 & 0 & & 0 \\
\hline 1922 & 12 & 1 & 2 & 0 & & 0 \\
\hline 1923 & 1 & 1 & 2 & 0 & & 0 \\
\hline 1923 & 2 & 1 & 2 & 0 & & 0 \\
\hline
\end{tabular}


Table III. (continued).

\begin{tabular}{|c|c|c|c|c|c|c|}
\hline year & month & $\begin{array}{c}\text { Etna } \\
\text { eruptions }\end{array}$ & VEI & $\begin{array}{l}\text { Stromboli } \\
\text { eruptions }\end{array}$ & VEI & $\begin{array}{c}\text { Vulcano } \\
\text { eruptions }\end{array}$ \\
\hline 1923 & 3 & 1 & 2 & 0 & & 0 \\
\hline 1923 & 4 & 1 & 2 & 0 & & 0 \\
\hline 1923 & 5 & 1 & 2 & 0 & & 0 \\
\hline 1923 & 6 & 1 & 2 & 0 & & 0 \\
\hline 1923 & 7 & 1 & 2 & 0 & & 0 \\
\hline 1930 & 2 & 0 & & 1 & 2 & 0 \\
\hline 1930 & 9 & 0 & & 1 & 3 & 0 \\
\hline 1930 & 10 & 0 & & 1 & 3 & 0 \\
\hline 1930 & 11 & 0 & & 1 & 3 & 0 \\
\hline 1930 & 12 & 0 & & 1 & 3 & 0 \\
\hline 1931 & 7 & 1 & 2 & 0 & & 0 \\
\hline 1931 & 8 & 1 & 2 & 0 & & 0 \\
\hline 1931 & 9 & 1 & 2 & 0 & & 0 \\
\hline 1931 & 10 & 1 & 2 & 0 & & 0 \\
\hline 1931 & 11 & 1 & 2 & 0 & & 0 \\
\hline 1931 & 12 & 1 & 2 & 0 & & 0 \\
\hline 1932 & 1 & 1 & 2 & 0 & & 0 \\
\hline 1932 & 2 & 1 & 2 & 0 & & 0 \\
\hline 1932 & 3 & 1 & 2 & 0 & & 0 \\
\hline 1932 & 4 & 1 & 2 & 0 & & 0 \\
\hline 1932 & 5 & 1 & 2 & 0 & & 0 \\
\hline 1932 & 6 & 1 & 2 & 0 & & 0 \\
\hline 1932 & 7 & 1 & 2 & 0 & & 0 \\
\hline 1932 & 8 & 1 & 2 & 0 & & 0 \\
\hline 1932 & 9 & 1 & 2 & 0 & & 0 \\
\hline 1932 & 10 & 1 & 2 & 0 & & 0 \\
\hline 1932 & 11 & 1 & 2 & 0 & & 0 \\
\hline 1932 & 12 & 1 & 2 & 0 & & 0 \\
\hline 1933 & 1 & 1 & 2 & 0 & & 0 \\
\hline 1933 & 2 & 1 & 2 & 0 & & 0 \\
\hline 1933 & 3 & 1 & 2 & 0 & & 0 \\
\hline 1933 & 4 & 1 & 2 & 0 & & 0 \\
\hline 1933 & 5 & 1 & 2 & 0 & & 0 \\
\hline 1933 & 6 & 1 & 2 & 0 & & 0 \\
\hline 1933 & 7 & 1 & 2 & 0 & & 0 \\
\hline 1933 & 8 & 1 & 2 & 0 & & 0 \\
\hline 1933 & 9 & 1 & 2 & 0 & & 0 \\
\hline 1934 & 2 & 0 & & 1 & 2 & 0 \\
\hline 1934 & 8 & 0 & & 1 & 2 & 0 \\
\hline 1935 & 7 & 1 & 2 & 0 & & 0 \\
\hline
\end{tabular}


Table III. (continued).

\begin{tabular}{|c|c|c|c|c|c|c|}
\hline year & month & $\begin{array}{c}\text { Etna } \\
\text { eruptions }\end{array}$ & VEI & $\begin{array}{l}\text { Stromboli } \\
\text { eruptions }\end{array}$ & VEI & $\begin{array}{c}\text { Vulcano } \\
\text { eruptions }\end{array}$ \\
\hline 1935 & 8 & 1 & 2 & 0 & & 0 \\
\hline 1935 & 9 & 1 & 2 & 0 & & 0 \\
\hline 1935 & 10 & 1 & 2 & 0 & & 0 \\
\hline 1935 & 11 & 1 & 2 & 0 & & 0 \\
\hline 1935 & 12 & 1 & 2 & 0 & & 0 \\
\hline 1936 & 1 & 1 & 2 & 1 & 2 & 0 \\
\hline 1936 & 2 & 1 & 2 & 0 & & 0 \\
\hline 1936 & 3 & 1 & 2 & 0 & & 0 \\
\hline 1936 & 4 & 1 & 2 & 0 & & 0 \\
\hline 1936 & 5 & 1 & 2 & 0 & & 0 \\
\hline 1936 & 6 & 1 & 2 & 0 & & 0 \\
\hline 1936 & 7 & 1 & 2 & 0 & & 0 \\
\hline 1936 & 8 & 1 & 2 & 0 & & 0 \\
\hline 1936 & 9 & 1 & 2 & 0 & & 0 \\
\hline 1936 & 10 & 1 & 2 & 0 & & 0 \\
\hline 1936 & 11 & 1 & 2 & 0 & & 0 \\
\hline 1936 & 12 & 1 & 2 & 0 & & 0 \\
\hline 1937 & 1 & 1 & 2 & 1 & 2 & 0 \\
\hline 1937 & 2 & 1 & 2 & 0 & & 0 \\
\hline 1937 & 3 & 1 & 2 & 0 & & 0 \\
\hline 1937 & 4 & 1 & 2 & 0 & & 0 \\
\hline 1937 & 5 & 1 & 2 & 0 & & 0 \\
\hline 1937 & 6 & 1 & 2 & 0 & & 0 \\
\hline 1937 & 7 & 1 & 2 & 0 & & 0 \\
\hline 1937 & 8 & 1 & 2 & 0 & & 0 \\
\hline 1937 & 9 & 1 & 2 & 0 & & 0 \\
\hline 1937 & 10 & 1 & 2 & 0 & & 0 \\
\hline 1937 & 11 & 1 & 2 & 1 & 2 & 0 \\
\hline 1937 & 12 & 1 & 2 & 0 & & 0 \\
\hline 1938 & 1 & 1 & 2 & 0 & & 0 \\
\hline 1938 & 2 & 1 & 2 & 0 & & 0 \\
\hline 1938 & 3 & 1 & 2 & 0 & & 0 \\
\hline 1938 & 4 & 1 & 2 & 0 & & 0 \\
\hline 1938 & 5 & 1 & 2 & 0 & & 0 \\
\hline 1938 & 6 & 1 & 2 & 0 & & 0 \\
\hline 1938 & 7 & 1 & 2 & 0 & & 0 \\
\hline 1938 & 8 & 1 & 2 & 0 & & 0 \\
\hline 1938 & 9 & 1 & 2 & 0 & & 0 \\
\hline 1938 & 10 & 1 & 2 & 0 & & 0 \\
\hline 1938 & 11 & 1 & 2 & 1 & 2 & 0 \\
\hline
\end{tabular}


Table III. (continued).

\begin{tabular}{|c|c|c|c|c|c|c|}
\hline year & month & $\begin{array}{c}\text { Etna } \\
\text { eruptions }\end{array}$ & VEI & $\begin{array}{l}\text { Stromboli } \\
\text { eruptions }\end{array}$ & VEI & $\begin{array}{c}\text { Vulcano } \\
\text { eruptions }\end{array}$ \\
\hline 1938 & 12 & 1 & 2 & 1 & 2 & 0 \\
\hline 1939 & 1 & 1 & 2 & 1 & 2 & 0 \\
\hline 1939 & 2 & 1 & 2 & 1 & 2 & 0 \\
\hline 1939 & 3 & 1 & 2 & 1 & 2 & 0 \\
\hline 1939 & 4 & 1 & 2 & 1 & 2 & 0 \\
\hline 1939 & 5 & 1 & 2 & 1 & 2 & 0 \\
\hline 1939 & 6 & 1 & 2 & 1 & 2 & 0 \\
\hline 1939 & 7 & 1 & 2 & 0 & & 0 \\
\hline 1939 & 8 & 1 & 2 & 0 & & 0 \\
\hline 1939 & 9 & 1 & 2 & 0 & & 0 \\
\hline 1939 & 10 & 1 & 2 & 0 & & 0 \\
\hline 1939 & 11 & 1 & 2 & 0 & & 0 \\
\hline 1939 & 12 & 1 & 2 & 0 & & 0 \\
\hline 1940 & 3 & 1 & 3 & 0 & & 0 \\
\hline 1940 & 4 & 1 & 3 & 0 & & 0 \\
\hline 1940 & 5 & 1 & 3 & 0 & & 0 \\
\hline 1940 & 6 & 1 & 3 & 0 & & 0 \\
\hline 1940 & 7 & 1 & 3 & 0 & & 0 \\
\hline 1940 & 8 & 1 & 3 & 0 & & 0 \\
\hline 1940 & 9 & 1 & 3 & 0 & & 0 \\
\hline 1940 & 10 & 1 & 3 & 0 & & 0 \\
\hline 1940 & 11 & 1 & 3 & 0 & & 0 \\
\hline 1940 & 12 & 1 & 3 & 0 & & 0 \\
\hline 1941 & 1 & 1 & 3 & 0 & & 0 \\
\hline 1941 & 2 & 1 & 3 & 0 & & 0 \\
\hline 1941 & 3 & 1 & 3 & 0 & & 0 \\
\hline 1941 & 4 & 1 & 3 & 0 & & 0 \\
\hline 1941 & 5 & 1 & 3 & 0 & & 0 \\
\hline 1941 & 6 & 1 & 3 & 0 & & 0 \\
\hline 1941 & 7 & 1 & 3 & 0 & & 0 \\
\hline 1941 & 8 & 1 & 3 & 1 & 2 & 0 \\
\hline 1941 & 9 & 1 & 3 & 0 & & 0 \\
\hline 1941 & 10 & 1 & 3 & 0 & & 0 \\
\hline 1941 & 11 & 1 & 3 & 0 & & 0 \\
\hline 1941 & 12 & 1 & 3 & 0 & & 0 \\
\hline 1942 & 1 & 1 & 3 & 0 & & 0 \\
\hline 1942 & 2 & 1 & 3 & 0 & & 0 \\
\hline 1942 & 3 & 1 & 3 & 0 & & 0 \\
\hline 1942 & 4 & 1 & 3 & 0 & & 0 \\
\hline 1942 & 5 & 1 & 3 & 0 & & 0 \\
\hline
\end{tabular}


Table III. (continued).

\begin{tabular}{|c|c|c|c|c|c|c|}
\hline year & month & $\begin{array}{c}\text { Etna } \\
\text { eruptions }\end{array}$ & VEI & $\begin{array}{l}\text { Stromboli } \\
\text { eruptions }\end{array}$ & VEI & $\begin{array}{c}\text { Vulcano } \\
\text { eruptions }\end{array}$ \\
\hline 1942 & 6 & 1 & 3 & 0 & & 0 \\
\hline 1942 & 7 & 1 & 2 & 0 & & 0 \\
\hline 1943 & 12 & 0 & & 1 & 2 & 0 \\
\hline 1944 & 1 & 0 & & 1 & 2 & 0 \\
\hline 1944 & 2 & 0 & & 1 & 2 & 0 \\
\hline 1944 & 8 & 0 & & 1 & 2 & 0 \\
\hline 1944 & 9 & 0 & & 1 & 2 & 0 \\
\hline 1944 & 10 & 0 & & 1 & 2 & 0 \\
\hline 1947 & 1 & 1 & 2 & 0 & & 0 \\
\hline 1947 & 2 & 1 & 2 & 0 & & 0 \\
\hline 1949 & 6 & 0 & & 1 & 2 & 0 \\
\hline 1949 & 12 & 1 & 2 & 0 & & 0 \\
\hline 1950 & 1 & 1 & 2 & 0 & & 0 \\
\hline 1950 & 10 & 0 & & 1 & 2 & 0 \\
\hline 1950 & 11 & 1 & 2 & 0 & & 0 \\
\hline 1950 & 12 & 1 & 2 & 0 & & 0 \\
\hline 1951 & 1 & 1 & 2 & 0 & & 0 \\
\hline 1951 & 2 & 1 & 2 & 0 & & 0 \\
\hline 1951 & 3 & 1 & 2 & 0 & & 0 \\
\hline 1951 & 4 & 1 & 2 & 1 & 2 & 0 \\
\hline 1951 & 5 & 1 & 2 & 0 & & 0 \\
\hline 1951 & 6 & 1 & 2 & 0 & & 0 \\
\hline 1951 & 7 & 1 & 2 & 0 & & 0 \\
\hline 1951 & 8 & 1 & 2 & 0 & & 0 \\
\hline 1951 & 9 & 1 & 2 & 0 & & 0 \\
\hline 1951 & 10 & 1 & 2 & 0 & & 0 \\
\hline 1951 & 11 & 1 & 2 & 0 & & 0 \\
\hline 1951 & 12 & 1 & 2 & 0 & & 0 \\
\hline 1952 & 6 & 0 & & 1 & 2 & 0 \\
\hline 1953 & 7 & 1 & 2 & 0 & & 0 \\
\hline 1954 & 2 & 0 & & 1 & 2 & 0 \\
\hline 1954 & 3 & 0 & & 1 & 2 & 0 \\
\hline 1954 & 12 & 0 & & 1 & 2 & 0 \\
\hline 1955 & 1 & 0 & & 1 & 2 & 0 \\
\hline 1955 & 2 & 0 & & 1 & 2 & 0 \\
\hline 1955 & 3 & 0 & & 1 & 2 & 0 \\
\hline 1955 & 4 & 1 & 2 & 1 & 2 & 0 \\
\hline 1955 & 5 & 1 & 2 & 1 & 2 & 0 \\
\hline 1955 & 6 & 1 & 2 & 0 & & 0 \\
\hline 1955 & 7 & 1 & 2 & 0 & & 0 \\
\hline
\end{tabular}


Table III. (continued).

\begin{tabular}{|c|c|c|c|c|c|c|}
\hline year & month & $\begin{array}{c}\text { Etna } \\
\text { eruptions }\end{array}$ & VEI & $\begin{array}{l}\text { Stromboli } \\
\text { eruptions }\end{array}$ & VEI & $\begin{array}{l}\text { Vulcano } \\
\text { eruptions }\end{array}$ \\
\hline 1955 & 8 & 1 & 2 & 0 & & 0 \\
\hline 1955 & 9 & 1 & 2 & 0 & & 0 \\
\hline 1955 & 10 & 1 & 2 & 0 & & 0 \\
\hline 1955 & 11 & 1 & 2 & 0 & & 0 \\
\hline 1955 & 12 & 1 & 2 & 0 & & 0 \\
\hline 1956 & 1 & 1 & 2 & 0 & & 0 \\
\hline 1956 & 2 & 1 & 2 & 0 & & 0 \\
\hline 1956 & 3 & 1 & 2 & 0 & & 0 \\
\hline 1956 & 4 & 1 & 2 & 0 & & 0 \\
\hline 1957 & 2 & 1 & 2 & 0 & & 0 \\
\hline 1957 & 3 & 1 & 2 & 0 & & 0 \\
\hline 1957 & 4 & 1 & 2 & 0 & & 0 \\
\hline 1957 & 5 & 1 & 2 & 0 & & 0 \\
\hline 1957 & 8 & 1 & 2 & 0 & & 0 \\
\hline 1957 & 9 & 1 & 2 & 0 & & 0 \\
\hline 1957 & 10 & 1 & 2 & 0 & & 0 \\
\hline 1957 & 11 & 1 & 2 & 0 & & 0 \\
\hline 1957 & 12 & 1 & 2 & 0 & & 0 \\
\hline 1958 & 1 & 1 & 2 & 0 & & 0 \\
\hline 1958 & 2 & 1 & 2 & 0 & & 0 \\
\hline 1958 & 3 & 1 & 2 & 0 & & 0 \\
\hline 1958 & 4 & 1 & 2 & 0 & & 0 \\
\hline 1958 & 5 & 1 & 2 & 0 & & 0 \\
\hline 1959 & 5 & 0 & & 1 & 2 & 0 \\
\hline 1959 & 10 & 1 & 3 & 0 & & 0 \\
\hline 1959 & 11 & 1 & 3 & 0 & & 0 \\
\hline 1959 & 12 & 1 & 3 & 0 & & 0 \\
\hline 1960 & 1 & 1 & 3 & 0 & & 0 \\
\hline 1960 & 2 & 1 & 3 & 0 & & 0 \\
\hline 1960 & 3 & 1 & 3 & 0 & & 0 \\
\hline 1960 & 4 & 1 & 3 & 0 & & 0 \\
\hline 1960 & 5 & 1 & 3 & 0 & & 0 \\
\hline 1960 & 6 & 1 & 3 & 0 & & 0 \\
\hline 1960 & 7 & 1 & 3 & 0 & & 0 \\
\hline 1960 & 8 & 1 & 3 & 0 & & 0 \\
\hline 1960 & 9 & 1 & 3 & 0 & & 0 \\
\hline 1960 & 10 & 1 & 3 & 0 & & 0 \\
\hline 1960 & 11 & 1 & 3 & 0 & & 0 \\
\hline 1960 & 12 & 1 & 3 & 0 & & 0 \\
\hline 1961 & 1 & 1 & 3 & 0 & & 0 \\
\hline
\end{tabular}


Table III. (continued).

\begin{tabular}{|c|c|c|c|c|c|c|}
\hline year & month & $\begin{array}{c}\text { Etna } \\
\text { eruptions }\end{array}$ & VEI & $\begin{array}{l}\text { Stromboli } \\
\text { eruptions }\end{array}$ & VEI & $\begin{array}{c}\text { Vulcano } \\
\text { eruptions }\end{array}$ \\
\hline 1961 & 2 & 1 & 3 & 0 & & 0 \\
\hline 1961 & 3 & 1 & 3 & 0 & & 0 \\
\hline 1961 & 4 & 1 & 3 & 0 & & 0 \\
\hline 1961 & 5 & 1 & 3 & 0 & & 0 \\
\hline 1961 & 6 & 1 & 3 & 0 & & 0 \\
\hline 1961 & 7 & 1 & 3 & 0 & & 0 \\
\hline 1961 & 8 & 1 & 3 & 0 & & 0 \\
\hline 1961 & 9 & 1 & 3 & 0 & & 0 \\
\hline 1961 & 10 & 1 & 3 & 0 & & 0 \\
\hline 1961 & 11 & 1 & 3 & 0 & & 0 \\
\hline 1961 & 12 & 1 & 3 & 0 & & 0 \\
\hline 1962 & 1 & 1 & 3 & 0 & & 0 \\
\hline 1962 & 2 & 1 & 3 & 0 & & 0 \\
\hline 1962 & 3 & 1 & 3 & 0 & & 0 \\
\hline 1962 & 4 & 1 & 3 & 0 & & 0 \\
\hline 1962 & 5 & 1 & 3 & 0 & & 0 \\
\hline 1962 & 6 & 1 & 3 & 0 & & 0 \\
\hline 1962 & 7 & 1 & 3 & 0 & & 0 \\
\hline 1962 & 8 & 1 & 3 & 0 & & 0 \\
\hline 1962 & 9 & 1 & 3 & 0 & & 0 \\
\hline 1962 & 10 & 1 & 3 & 0 & & 0 \\
\hline 1962 & 11 & 1 & 3 & 0 & & 0 \\
\hline 1962 & 12 & 1 & 3 & 0 & & 0 \\
\hline 1963 & 1 & 1 & 3 & 0 & & 0 \\
\hline 1963 & 2 & 1 & 3 & 0 & & 0 \\
\hline 1963 & 3 & 1 & 3 & 0 & & 0 \\
\hline 1963 & 4 & 1 & 3 & 0 & & 0 \\
\hline 1963 & 5 & 1 & 3 & 0 & & 0 \\
\hline 1963 & 6 & 1 & 3 & 0 & & 0 \\
\hline 1963 & 7 & 1 & 3 & 0 & & 0 \\
\hline 1963 & 8 & 1 & 3 & 0 & & 0 \\
\hline 1963 & 9 & 1 & 3 & 0 & & 0 \\
\hline 1963 & 10 & 1 & 3 & 0 & & 0 \\
\hline 1963 & 11 & 1 & 3 & 0 & & 0 \\
\hline 1963 & 12 & 1 & 3 & 0 & & 0 \\
\hline 1964 & 1 & 1 & 3 & 0 & & 0 \\
\hline 1964 & 2 & 1 & 3 & 0 & & 0 \\
\hline 1964 & 3 & 1 & 3 & 0 & & 0 \\
\hline 1964 & 4 & 1 & 3 & 0 & & 0 \\
\hline 1964 & 5 & 1 & 3 & 0 & & 0 \\
\hline
\end{tabular}


Table III. (continued).

\begin{tabular}{|c|c|c|c|c|c|c|}
\hline year & month & $\begin{array}{c}\text { Etna } \\
\text { eruptions }\end{array}$ & VEI & $\begin{array}{l}\text { Stromboli } \\
\text { eruptions }\end{array}$ & VEI & $\begin{array}{c}\text { Vulcano } \\
\text { eruptions }\end{array}$ \\
\hline 1964 & 6 & 1 & 3 & 0 & & 0 \\
\hline 1964 & 7 & 1 & 3 & 0 & & 0 \\
\hline 1964 & 8 & 1 & 3 & 0 & & 0 \\
\hline 1964 & 9 & 1 & 3 & 0 & & 0 \\
\hline 1964 & 10 & 1 & 3 & 0 & & 0 \\
\hline 1964 & 11 & 1 & 3 & 0 & & 0 \\
\hline 1964 & 12 & 1 & 3 & 0 & & 0 \\
\hline 1966 & 1 & 1 & 2 & 0 & & 0 \\
\hline 1966 & 2 & 1 & 2 & 0 & & 0 \\
\hline 1966 & 3 & 1 & 2 & 0 & & 0 \\
\hline 1966 & 4 & 1 & 2 & 1 & 2 & 0 \\
\hline 1966 & 5 & 1 & 2 & 0 & & 0 \\
\hline 1966 & 6 & 1 & 2 & 0 & & 0 \\
\hline 1966 & 7 & 1 & 2 & 0 & & 0 \\
\hline 1966 & 8 & 1 & 2 & 0 & & 0 \\
\hline 1966 & 9 & 1 & 2 & 0 & & 0 \\
\hline 1966 & 10 & 1 & 2 & 0 & & 0 \\
\hline 1966 & 11 & 1 & 2 & 0 & & 0 \\
\hline 1966 & 12 & 1 & 2 & 0 & & 0 \\
\hline 1967 & 1 & 1 & 2 & 0 & & 0 \\
\hline 1967 & 2 & 1 & 2 & 0 & & 0 \\
\hline 1967 & 3 & 1 & 2 & 0 & & 0 \\
\hline 1967 & 4 & 1 & 2 & 0 & & 0 \\
\hline 1967 & 5 & 1 & 2 & 0 & & 0 \\
\hline 1967 & 6 & 1 & 2 & 0 & & 0 \\
\hline 1967 & 7 & 1 & 2 & 0 & & 0 \\
\hline 1967 & 8 & 1 & 2 & 0 & & 0 \\
\hline 1967 & 9 & 1 & 2 & 0 & & 0 \\
\hline 1967 & 10 & 1 & 2 & 0 & & 0 \\
\hline 1967 & 11 & 1 & 2 & 0 & & 0 \\
\hline 1967 & 12 & 1 & 2 & 0 & & 0 \\
\hline 1968 & 1 & 1 & 2 & 0 & & 0 \\
\hline 1968 & 2 & 1 & 2 & 0 & & 0 \\
\hline 1968 & 3 & 1 & 2 & 0 & & 0 \\
\hline 1968 & 4 & 1 & 2 & 0 & & 0 \\
\hline 1968 & 5 & 1 & 2 & 0 & & 0 \\
\hline 1968 & 6 & 1 & 2 & 0 & & 0 \\
\hline 1968 & 7 & 1 & 2 & 0 & & 0 \\
\hline 1968 & 8 & 1 & 2 & 0 & & 0 \\
\hline 1968 & 9 & 1 & 2 & 0 & & 0 \\
\hline
\end{tabular}


Table III. (continued).

\begin{tabular}{|c|c|c|c|c|c|c|}
\hline year & month & $\begin{array}{c}\text { Etna } \\
\text { eruptions }\end{array}$ & VEI & $\begin{array}{l}\text { Stromboli } \\
\text { eruptions }\end{array}$ & VEI & $\begin{array}{c}\text { Vulcano } \\
\text { eruptions }\end{array}$ \\
\hline 1968 & 10 & 1 & 2 & 0 & & 0 \\
\hline 1968 & 11 & 1 & 2 & 0 & & 0 \\
\hline 1968 & 12 & 1 & 2 & 0 & & 0 \\
\hline 1969 & 1 & 1 & 2 & 0 & & 0 \\
\hline 1969 & 2 & 1 & 2 & 0 & & 0 \\
\hline 1969 & 3 & 1 & 2 & 0 & & 0 \\
\hline 1969 & 4 & 1 & 2 & 0 & & 0 \\
\hline 1969 & 5 & 1 & 2 & 0 & & 0 \\
\hline 1969 & 6 & 1 & 2 & 0 & & 0 \\
\hline 1969 & 7 & 1 & 2 & 0 & & 0 \\
\hline 1969 & 8 & 1 & 2 & 0 & & 0 \\
\hline 1969 & 9 & 1 & 2 & 0 & & 0 \\
\hline 1969 & 10 & 1 & 2 & 0 & & 0 \\
\hline 1969 & 11 & 1 & 2 & 0 & & 0 \\
\hline 1969 & 12 & 1 & 2 & 0 & & 0 \\
\hline 1970 & 1 & 1 & 2 & 0 & & 0 \\
\hline 1970 & 2 & 1 & 2 & 0 & & 0 \\
\hline 1970 & 3 & 1 & 2 & 0 & & 0 \\
\hline 1970 & 4 & 1 & 2 & 0 & & 0 \\
\hline 1970 & 5 & 1 & 2 & 0 & & 0 \\
\hline 1970 & 6 & 1 & 2 & 0 & & 0 \\
\hline 1970 & 7 & 1 & 2 & 0 & & 0 \\
\hline 1970 & 8 & 1 & 2 & 0 & & 0 \\
\hline 1970 & 9 & 1 & 2 & 0 & & 0 \\
\hline 1970 & 10 & 1 & 2 & 0 & & 0 \\
\hline 1970 & 11 & 1 & 2 & 0 & & 0 \\
\hline 1970 & 12 & 1 & 2 & 0 & & 0 \\
\hline 1971 & 1 & 1 & 2 & 0 & & 0 \\
\hline 1971 & 3 & 0 & & 1 & 2 & 0 \\
\hline 1971 & 4 & 1 & 2 & 1 & 2 & 0 \\
\hline 1971 & 5 & 1 & 2 & 1 & 2 & 0 \\
\hline 1971 & 6 & 1 & 2 & 0 & & 0 \\
\hline 1971 & 9 & 1 & 2 & 0 & & 0 \\
\hline 1971 & 10 & 1 & 2 & 0 & & 0 \\
\hline 1971 & 11 & 1 & 2 & 0 & & 0 \\
\hline 1971 & 12 & 1 & 2 & 0 & & 0 \\
\hline 1972 & 1 & 1 & 2 & 0 & & 0 \\
\hline 1972 & 2 & 1 & 2 & 0 & & 0 \\
\hline 1972 & 3 & 1 & 2 & 0 & & 0 \\
\hline 1972 & 4 & 1 & 2 & 0 & & 0 \\
\hline
\end{tabular}


Table III. (continued).

\begin{tabular}{|c|c|c|c|c|c|c|}
\hline year & month & $\begin{array}{c}\text { Etna } \\
\text { eruptions }\end{array}$ & VEI & $\begin{array}{l}\text { Stromboli } \\
\text { eruptions }\end{array}$ & VEI & $\begin{array}{c}\text { Vulcano } \\
\text { eruptions }\end{array}$ \\
\hline 1972 & 5 & 1 & 2 & 0 & & 0 \\
\hline 1972 & 6 & 1 & 2 & 0 & & 0 \\
\hline 1972 & 7 & 1 & 2 & 0 & & 0 \\
\hline 1972 & 8 & 1 & 2 & 0 & & 0 \\
\hline 1972 & 9 & 1 & 2 & 0 & & 0 \\
\hline 1972 & 10 & 1 & 2 & 0 & & 0 \\
\hline 1972 & 11 & 1 & 2 & 0 & & 0 \\
\hline 1972 & 12 & 1 & 2 & 0 & & 0 \\
\hline 1973 & 1 & 1 & 2 & 0 & & 0 \\
\hline 1973 & 2 & 1 & 2 & 0 & & 0 \\
\hline 1973 & 3 & 1 & 2 & 0 & & 0 \\
\hline 1973 & 4 & 1 & 2 & 0 & & 0 \\
\hline 1973 & 5 & 1 & 2 & 0 & & 0 \\
\hline 1973 & 6 & 1 & 2 & 0 & & 0 \\
\hline 1973 & 7 & 1 & 2 & 0 & & 0 \\
\hline 1973 & 8 & 1 & 2 & 0 & & 0 \\
\hline 1973 & 9 & 1 & 2 & 0 & & 0 \\
\hline 1973 & 10 & 1 & 2 & 0 & & 0 \\
\hline 1973 & 11 & 1 & 2 & 0 & & 0 \\
\hline 1973 & 12 & 1 & 2 & 0 & & 0 \\
\hline 1974 & 1 & 1 & 2 & 0 & & 0 \\
\hline 1974 & 2 & 1 & 2 & 0 & & 0 \\
\hline 1974 & 3 & 1 & 2 & 0 & & 0 \\
\hline 1974 & 4 & 1 & 2 & 0 & & 0 \\
\hline 1974 & 5 & 1 & 2 & 0 & & 0 \\
\hline 1974 & 6 & 1 & 2 & 0 & & 0 \\
\hline 1974 & 7 & 1 & 2 & 0 & & 0 \\
\hline 1974 & 8 & 1 & 2 & 0 & & 0 \\
\hline 1974 & 9 & 1 & 2 & 0 & & 0 \\
\hline 1974 & 10 & 1 & 2 & 0 & & 0 \\
\hline 1974 & 11 & 1 & 2 & 0 & & 0 \\
\hline 1974 & 12 & 1 & 2 & 0 & & 0 \\
\hline 1975 & 1 & 1 & 2 & 0 & & 0 \\
\hline 1975 & 2 & 1 & 2 & 0 & & 0 \\
\hline 1975 & 3 & 1 & 2 & 0 & & 0 \\
\hline 1975 & 4 & 1 & 2 & 0 & & 0 \\
\hline 1975 & 5 & 1 & 2 & 0 & & 0 \\
\hline 1975 & 6 & 1 & 2 & 0 & & 0 \\
\hline 1975 & 7 & 1 & 2 & 0 & & 0 \\
\hline 1975 & 8 & 1 & 2 & 0 & & 0 \\
\hline
\end{tabular}


Table III. (continued).

\begin{tabular}{|c|c|c|c|c|c|c|}
\hline year & month & $\begin{array}{c}\text { Etna } \\
\text { eruptions }\end{array}$ & VEI & $\begin{array}{l}\text { Stromboli } \\
\text { eruptions }\end{array}$ & VEI & $\begin{array}{c}\text { Vulcano } \\
\text { eruptions }\end{array}$ \\
\hline 1975 & 9 & 1 & 2 & 0 & & 0 \\
\hline 1975 & 10 & 1 & 2 & 0 & & 0 \\
\hline 1975 & 11 & 1 & 2 & 0 & & 0 \\
\hline 1975 & 12 & 1 & 2 & 0 & & 0 \\
\hline 1976 & 1 & 1 & 2 & 0 & & 0 \\
\hline 1976 & 2 & 1 & 2 & 0 & & 0 \\
\hline 1976 & 3 & 1 & 2 & 0 & & 0 \\
\hline 1976 & 4 & 1 & 2 & 0 & & 0 \\
\hline 1976 & 5 & 1 & 2 & 0 & & 0 \\
\hline 1976 & 6 & 1 & 2 & 0 & & 0 \\
\hline 1976 & 7 & 1 & 2 & 0 & & 0 \\
\hline 1976 & 8 & 1 & 2 & 0 & & 0 \\
\hline 1976 & 9 & 1 & 2 & 0 & & 0 \\
\hline 1976 & 10 & 1 & 2 & 0 & & 0 \\
\hline 1976 & 11 & 1 & 2 & 0 & & 0 \\
\hline 1976 & 12 & 1 & 2 & 0 & & 0 \\
\hline 1977 & 1 & 1 & 2 & 0 & & 0 \\
\hline 1977 & 2 & 1 & 2 & 0 & & 0 \\
\hline 1977 & 3 & 1 & 2 & 0 & & 0 \\
\hline 1977 & 4 & 1 & 2 & 0 & & 0 \\
\hline 1977 & 5 & 1 & 2 & 0 & & 0 \\
\hline 1977 & 6 & 1 & 2 & 0 & & 0 \\
\hline 1977 & 7 & 1 & 2 & 0 & & 0 \\
\hline 1977 & 8 & 1 & 2 & 0 & & 0 \\
\hline 1977 & 9 & 1 & 2 & 0 & & 0 \\
\hline 1977 & 10 & 1 & 2 & 0 & & 0 \\
\hline 1977 & 11 & 1 & 2 & 0 & & 0 \\
\hline 1977 & 12 & 1 & 2 & 0 & & 0 \\
\hline 1978 & 1 & 1 & 2 & 0 & & 0 \\
\hline 1978 & 2 & 1 & 2 & 0 & & 0 \\
\hline 1978 & 3 & 1 & 2 & 0 & & 0 \\
\hline 1978 & 4 & 1 & 2 & 0 & & 0 \\
\hline 1978 & 5 & 1 & 2 & 0 & & 0 \\
\hline 1978 & 6 & 1 & 2 & 0 & & 0 \\
\hline 1978 & 7 & 1 & 2 & 0 & & 0 \\
\hline 1978 & 8 & 1 & 2 & 0 & & 0 \\
\hline 1978 & 9 & 1 & 2 & 0 & & 0 \\
\hline 1978 & 10 & 1 & 2 & 0 & & 0 \\
\hline 1978 & 11 & 1 & 2 & 0 & & 0 \\
\hline 1978 & 12 & 1 & 2 & 0 & & 0 \\
\hline
\end{tabular}


Table III. (continued).

\begin{tabular}{|c|c|c|c|c|c|c|}
\hline year & month & $\begin{array}{c}\text { Etna } \\
\text { eruptions }\end{array}$ & VEI & $\begin{array}{l}\text { Stromboli } \\
\text { eruptions }\end{array}$ & VEI & $\begin{array}{c}\text { Vulcano } \\
\text { eruptions }\end{array}$ \\
\hline 1979 & 1 & 1 & 2 & 0 & & 0 \\
\hline 1979 & 2 & 1 & 2 & 0 & & 0 \\
\hline 1979 & 3 & 1 & 2 & 0 & & 0 \\
\hline 1979 & 7 & 1 & 2 & 0 & & 0 \\
\hline 1979 & 8 & 1 & 2 & 0 & & 0 \\
\hline 1979 & 9 & 1 & 2 & 0 & & 0 \\
\hline 1979 & 10 & 1 & 2 & 0 & & 0 \\
\hline 1979 & 11 & 1 & 2 & 0 & & 0 \\
\hline 1979 & 12 & 1 & 2 & 0 & & 0 \\
\hline 1980 & 1 & 1 & 2 & 0 & & 0 \\
\hline 1980 & 2 & 1 & 2 & 0 & & 0 \\
\hline 1980 & 3 & 1 & 2 & 0 & & 0 \\
\hline 1980 & 4 & 1 & 2 & 0 & & 0 \\
\hline 1980 & 5 & 1 & 2 & 0 & & 0 \\
\hline 1980 & 6 & 1 & 2 & 0 & & 0 \\
\hline 1980 & 7 & 1 & 3 & 0 & & 0 \\
\hline 1980 & 8 & 1 & 3 & 0 & & 0 \\
\hline 1980 & 9 & 1 & 3 & 0 & & 0 \\
\hline 1980 & 10 & 1 & 2 & 0 & & 0 \\
\hline 1980 & 11 & 1 & 2 & 0 & & 0 \\
\hline 1980 & 12 & 1 & 2 & 0 & & 0 \\
\hline 1981 & 1 & 1 & 2 & 0 & & 0 \\
\hline 1981 & 2 & 1 & 2 & 0 & & 0 \\
\hline 1981 & 3 & 1 & 2 & 0 & & 0 \\
\hline 1981 & 4 & 1 & 2 & 0 & & 0 \\
\hline 1981 & 5 & 1 & 2 & 0 & & 0 \\
\hline 1981 & 6 & 1 & 2 & 0 & & 0 \\
\hline 1981 & 7 & 1 & 2 & 0 & & 0 \\
\hline 1981 & 8 & 1 & 2 & 0 & & 0 \\
\hline 1981 & 9 & 1 & 2 & 0 & & 0 \\
\hline 1981 & 10 & 1 & 2 & 0 & & 0 \\
\hline 1981 & 11 & 1 & 2 & 0 & & 0 \\
\hline 1981 & 12 & 1 & 2 & 0 & & 0 \\
\hline 1982 & 1 & 1 & 2 & 0 & & 0 \\
\hline 1982 & 2 & 1 & 2 & 0 & & 0 \\
\hline 1982 & 3 & 1 & 2 & 0 & & 0 \\
\hline 1982 & 4 & 1 & 2 & 0 & & 0 \\
\hline 1982 & 5 & 1 & 2 & 0 & & 0 \\
\hline 1982 & 6 & 1 & 2 & 0 & & 0 \\
\hline 1982 & 7 & 1 & 2 & 0 & & 0 \\
\hline
\end{tabular}


Table III. (continued).

\begin{tabular}{|c|c|c|c|c|c|c|}
\hline year & month & $\begin{array}{c}\text { Etna } \\
\text { eruptions }\end{array}$ & VEI & $\begin{array}{l}\text { Stromboli } \\
\text { eruptions }\end{array}$ & VEI & $\begin{array}{c}\text { Vulcano } \\
\text { eruptions }\end{array}$ \\
\hline 1982 & 8 & 1 & 2 & 0 & & 0 \\
\hline 1982 & 9 & 1 & 2 & 0 & & 0 \\
\hline 1982 & 10 & 1 & 2 & 0 & & 0 \\
\hline 1982 & 11 & 1 & 2 & 0 & & 0 \\
\hline 1982 & 12 & 1 & 2 & 0 & & 0 \\
\hline 1983 & 1 & 1 & 2 & 0 & & 0 \\
\hline 1983 & 2 & 1 & 2 & 0 & & 0 \\
\hline 1983 & 3 & 1 & 2 & 0 & & 0 \\
\hline 1983 & 4 & 1 & 2 & 0 & & 0 \\
\hline 1983 & 5 & 1 & 2 & 0 & & 0 \\
\hline 1983 & 6 & 1 & 2 & 0 & & 0 \\
\hline 1983 & 7 & 1 & 2 & 0 & & 0 \\
\hline 1983 & 8 & 1 & 2 & 0 & & 0 \\
\hline 1983 & 9 & 1 & 2 & 0 & & 0 \\
\hline 1983 & 10 & 1 & 2 & 0 & & 0 \\
\hline 1983 & 11 & 1 & 2 & 0 & & 0 \\
\hline 1983 & 12 & 1 & 2 & 0 & & 0 \\
\hline 1984 & 1 & 1 & 2 & 0 & & 0 \\
\hline 1984 & 2 & 1 & 2 & 0 & & 0 \\
\hline 1984 & 3 & 1 & 2 & 0 & & 0 \\
\hline 1984 & 4 & 1 & 2 & 0 & & 0 \\
\hline 1984 & 5 & 1 & 2 & 0 & & 0 \\
\hline 1984 & 6 & 1 & 2 & 0 & & 0 \\
\hline 1984 & 7 & 1 & 2 & 0 & & 0 \\
\hline 1984 & 8 & 1 & 2 & 0 & & 0 \\
\hline 1984 & 9 & 1 & 2 & 0 & & 0 \\
\hline 1984 & 10 & 1 & 2 & 0 & & 0 \\
\hline 1984 & 11 & 1 & 2 & 0 & & 0 \\
\hline 1984 & 12 & 1 & 2 & 0 & & 0 \\
\hline 1985 & 1 & 1 & 2 & 0 & & 0 \\
\hline 1985 & 2 & 1 & 2 & 0 & & 0 \\
\hline 1985 & 3 & 1 & 2 & 0 & & 0 \\
\hline 1985 & 4 & 1 & 2 & 0 & & 0 \\
\hline 1985 & 5 & 1 & 2 & 0 & & 0 \\
\hline 1985 & 6 & 1 & 2 & 0 & & 0 \\
\hline 1985 & 7 & 1 & 2 & 0 & & 0 \\
\hline 1985 & 8 & 1 & 2 & 0 & & 0 \\
\hline 1985 & 9 & 1 & 2 & 0 & & 0 \\
\hline 1985 & 10 & 1 & 2 & 0 & & 0 \\
\hline 1985 & 11 & 1 & 2 & 0 & & 0 \\
\hline
\end{tabular}


Table III. (continued).

\begin{tabular}{|c|c|c|c|c|c|c|}
\hline year & month & $\begin{array}{c}\text { Etna } \\
\text { eruptions }\end{array}$ & VEI & $\begin{array}{l}\text { Stromboli } \\
\text { eruptions }\end{array}$ & VEI & $\begin{array}{c}\text { Vulcano } \\
\text { eruptions }\end{array}$ \\
\hline 1985 & 12 & 1 & 2 & 1 & 2 & 0 \\
\hline 1986 & 1 & 1 & 2 & 1 & 2 & 0 \\
\hline 1986 & 2 & 1 & 2 & 1 & 2 & 0 \\
\hline 1986 & 3 & 1 & 2 & 1 & 2 & 0 \\
\hline 1986 & 4 & 1 & 2 & 1 & 2 & 0 \\
\hline 1986 & 5 & 1 & 2 & 0 & & 0 \\
\hline 1986 & 6 & 1 & 2 & 0 & & 0 \\
\hline 1986 & 7 & 1 & 2 & 0 & & 0 \\
\hline 1986 & 8 & 1 & 2 & 0 & & 0 \\
\hline 1986 & 9 & 1 & 2 & 0 & & 0 \\
\hline 1986 & 10 & 1 & 2 & 0 & & 0 \\
\hline 1986 & 11 & 1 & 2 & 0 & & 0 \\
\hline 1986 & 12 & 1 & 2 & 0 & & 0 \\
\hline 1987 & 1 & 1 & 2 & 0 & & 0 \\
\hline 1987 & 2 & 1 & 2 & 0 & & 0 \\
\hline 1987 & 3 & 1 & 2 & 0 & & 0 \\
\hline 1987 & 4 & 1 & 2 & 0 & & 0 \\
\hline 1987 & 5 & 1 & 2 & 0 & & 0 \\
\hline 1987 & 6 & 1 & 2 & 0 & & 0 \\
\hline 1987 & 7 & 1 & 2 & 0 & & 0 \\
\hline 1987 & 8 & 1 & 2 & 0 & & 0 \\
\hline 1987 & 9 & 1 & 2 & 0 & & 0 \\
\hline 1987 & 10 & 1 & 2 & 0 & & 0 \\
\hline 1987 & 11 & 1 & 2 & 0 & & 0 \\
\hline 1987 & 12 & 1 & 2 & 0 & & 0 \\
\hline 1988 & 1 & 1 & 2 & 0 & & 0 \\
\hline 1988 & 2 & 1 & 2 & 0 & & 0 \\
\hline 1988 & 3 & 1 & 2 & 0 & & 0 \\
\hline 1988 & 4 & 1 & 2 & 0 & & 0 \\
\hline 1988 & 5 & 1 & 2 & 0 & & 0 \\
\hline 1988 & 6 & 1 & 2 & 0 & & 0 \\
\hline 1988 & 7 & 1 & 2 & 0 & & 0 \\
\hline 1988 & 8 & 1 & 2 & 0 & & 0 \\
\hline 1988 & 9 & 1 & 2 & 0 & & 0 \\
\hline 1988 & 10 & 1 & 2 & 0 & & 0 \\
\hline 1988 & 11 & 1 & 2 & 0 & & 0 \\
\hline 1988 & 12 & 1 & 2 & 0 & & 0 \\
\hline 1989 & 1 & 1 & 2 & 0 & & 0 \\
\hline 1989 & 2 & 1 & 2 & 0 & & 0 \\
\hline 1989 & 3 & 1 & 2 & 0 & & 0 \\
\hline
\end{tabular}


Table III. (continued).

\begin{tabular}{|c|c|c|c|c|c|c|}
\hline year & month & $\begin{array}{c}\text { Etna } \\
\text { eruptions }\end{array}$ & VEI & $\begin{array}{l}\text { Stromboli } \\
\text { eruptions }\end{array}$ & VEI & $\begin{array}{c}\text { Vulcano } \\
\text { eruptions }\end{array}$ \\
\hline 1989 & 4 & 1 & 2 & 0 & & 0 \\
\hline 1989 & 5 & 1 & 2 & 0 & & 0 \\
\hline 1989 & 6 & 1 & 2 & 0 & & 0 \\
\hline 1989 & 7 & 1 & 2 & 0 & & 0 \\
\hline 1989 & 8 & 1 & 2 & 0 & & 0 \\
\hline 1989 & 9 & 1 & 2 & 0 & & 0 \\
\hline 1989 & 10 & 1 & 2 & 0 & & 0 \\
\hline 1989 & 11 & 1 & 2 & 0 & & 0 \\
\hline 1989 & 12 & 1 & 2 & 0 & & 0 \\
\hline 1990 & 1 & 1 & 2 & 0 & & 0 \\
\hline 1990 & 2 & 1 & 2 & 0 & & 0 \\
\hline 1990 & 3 & 1 & 2 & 0 & & 0 \\
\hline 1990 & 4 & 1 & 2 & 0 & & 0 \\
\hline 1990 & 5 & 1 & 2 & 0 & & 0 \\
\hline 1990 & 6 & 1 & 2 & 0 & & 0 \\
\hline 1990 & 7 & 1 & 2 & 0 & & 0 \\
\hline 1990 & 8 & 1 & 2 & 0 & & 0 \\
\hline 1990 & 9 & 1 & 2 & 0 & & 0 \\
\hline 1990 & 10 & 1 & 2 & 0 & & 0 \\
\hline 1990 & 11 & 1 & 2 & 0 & & 0 \\
\hline 1990 & 12 & 1 & 2 & 0 & & 0 \\
\hline 1991 & 1 & 1 & 2 & 0 & & 0 \\
\hline 1991 & 2 & 1 & 2 & 0 & & 0 \\
\hline 1991 & 3 & 1 & 2 & 0 & & 0 \\
\hline 1991 & 4 & 1 & 2 & 0 & & 0 \\
\hline 1991 & 5 & 1 & 2 & 0 & & 0 \\
\hline 1991 & 6 & 1 & 2 & 0 & & 0 \\
\hline 1991 & 7 & 1 & 2 & 0 & & 0 \\
\hline 1991 & 8 & 1 & 2 & 0 & & 0 \\
\hline 1991 & 9 & 1 & 2 & 0 & & 0 \\
\hline 1991 & 10 & 1 & 2 & 0 & & 0 \\
\hline 1991 & 11 & 1 & 2 & 0 & & 0 \\
\hline 1991 & 12 & 1 & 2 & 0 & & 0 \\
\hline 1992 & 1 & 1 & 2 & 0 & & 0 \\
\hline 1992 & 2 & 1 & 2 & 0 & & 0 \\
\hline 1992 & 3 & 1 & 2 & 0 & & 0 \\
\hline 1992 & 4 & 1 & 2 & 0 & & 0 \\
\hline 1992 & 5 & 1 & 2 & 0 & & 0 \\
\hline 1992 & 6 & 1 & 2 & 0 & & 0 \\
\hline 1992 & 7 & 1 & 2 & 0 & & 0 \\
\hline
\end{tabular}


Table III. (continued).

\begin{tabular}{|c|c|c|c|c|c|c|}
\hline year & month & $\begin{array}{c}\text { Etna } \\
\text { eruptions }\end{array}$ & VEI & $\begin{array}{l}\text { Stromboli } \\
\text { eruptions }\end{array}$ & VEI & $\begin{array}{c}\text { Vulcano } \\
\text { eruptions }\end{array}$ \\
\hline 1992 & 8 & 1 & 2 & 0 & & 0 \\
\hline 1992 & 9 & 1 & 2 & 0 & & 0 \\
\hline 1992 & 10 & 1 & 2 & 0 & & 0 \\
\hline 1992 & 11 & 1 & 2 & 0 & & 0 \\
\hline 1992 & 12 & 1 & 2 & 0 & & 0 \\
\hline 1993 & 1 & 1 & 2 & 0 & & 0 \\
\hline 1993 & 2 & 1 & 2 & 0 & & 0 \\
\hline 1993 & 3 & 1 & 2 & 0 & & 0 \\
\hline
\end{tabular}

Table IV.Eruption (with VEI $\geq 2$ ) occurrences by year between 0 and 1993 AD at Etna, Stromboli, and Vulcano. VEI $=$ Volcanic Explosivity Index; 0 = no occurrence; 1 = occurrence. Data are from Simkin and Siebert (1994).

\begin{tabular}{|c|c|c|c|c|c|c|}
\hline year & $\begin{array}{c}\text { Etna } \\
\text { eruptions }\end{array}$ & VEI & $\begin{array}{l}\text { Stromboli } \\
\text { eruptions }\end{array}$ & VEI & $\begin{array}{l}\text { Vulcano } \\
\text { eruptions }\end{array}$ & VEI \\
\hline 252 & 1 & 3 & 0 & & 0 & \\
\hline 526 & 0 & & 0 & & 1 & 3 \\
\hline 925 & 0 & & 0 & & 1 & 3 \\
\hline 1157 & 1 & 2 & 0 & & 0 & \\
\hline 1160 & 1 & 2 & 0 & & 0 & \\
\hline 1164 & 1 & 2 & 0 & & 0 & \\
\hline 1194 & 1 & 2 & 0 & & 0 & \\
\hline 1222 & 1 & 2 & 0 & & 0 & \\
\hline 1250 & 1 & 2 & 0 & & 0 & \\
\hline 1329 & 1 & 3 & 0 & & 0 & \\
\hline 1333 & 1 & 2 & 0 & & 0 & \\
\hline 1350 & 1 & 2 & 0 & & 0 & \\
\hline 1381 & 1 & 2 & 0 & & 0 & \\
\hline 1408 & 1 & 3 & 0 & & 0 & \\
\hline 1444 & 1 & 2 & 0 & & 1 & 3 \\
\hline 1536 & 1 & 3 & 0 & & 0 & \\
\hline 1537 & 1 & 2 & 0 & & 0 & \\
\hline 1541 & 1 & 2 & 0 & & 0 & \\
\hline 1550 & 0 & & 0 & & 1 & 3 \\
\hline 1554 & 1 & 2 & 0 & & 0 & \\
\hline 1558 & 0 & & 1 & 2 & 0 & \\
\hline 1566 & 1 & 2 & 0 & & 0 & \\
\hline 1595 & 1 & 3 & 0 & & 0 & \\
\hline
\end{tabular}


Table IV.(continued).

\begin{tabular}{|c|c|c|c|c|c|c|}
\hline year & $\begin{array}{c}\text { Etna } \\
\text { eruptions }\end{array}$ & VEI & $\begin{array}{l}\text { Stromboli } \\
\text { eruptions }\end{array}$ & VEI & $\begin{array}{l}\text { Vulcano } \\
\text { eruptions }\end{array}$ & VEI \\
\hline 1603 & 1 & 2 & 0 & & 0 & \\
\hline 1607 & 1 & 2 & 0 & & 0 & \\
\hline 1610 & 1 & 2 & 0 & & 0 & \\
\hline 1614 & 1 & 2 & 0 & & 0 & \\
\hline 1615 & 1 & 2 & 0 & & 0 & \\
\hline 1616 & 1 & 2 & 0 & & 0 & \\
\hline 1617 & 1 & 2 & 0 & & 0 & \\
\hline 1618 & 1 & 2 & 0 & & 0 & \\
\hline 1619 & 1 & 2 & 0 & & 0 & \\
\hline 1620 & 1 & 2 & 0 & & 0 & \\
\hline 1621 & 1 & 2 & 0 & & 0 & \\
\hline 1622 & 1 & 2 & 0 & & 0 & \\
\hline 1623 & 1 & 2 & 0 & & 0 & \\
\hline 1624 & 1 & 2 & 0 & & 0 & \\
\hline 1626 & 0 & & 0 & & 1 & 3 \\
\hline 1646 & 1 & 2 & 0 & & 0 & \\
\hline 1647 & 1 & 2 & 0 & & 0 & \\
\hline 1669 & 1 & 3 & 0 & & 0 & \\
\hline 1682 & 1 & 2 & 0 & & 0 & \\
\hline 1693 & 1 & 3 & 0 & & 0 & \\
\hline 1694 & 1 & 3 & 0 & & 0 & \\
\hline 1723 & 1 & 2 & 0 & & 0 & \\
\hline 1724 & 1 & 2 & 0 & & 0 & \\
\hline 1727 & 0 & & 0 & & 1 & 3 \\
\hline 1731 & 0 & & 0 & & 1 & 3 \\
\hline 1732 & 1 & 2 & 0 & & 1 & 3 \\
\hline 1733 & 1 & 2 & 0 & & 1 & 3 \\
\hline 1734 & 0 & & 0 & & 1 & 3 \\
\hline 1735 & 1 & 2 & 0 & & 1 & 3 \\
\hline 1736 & 1 & 2 & 0 & & 1 & 3 \\
\hline 1737 & 0 & & 0 & & 1 & 3 \\
\hline 1738 & 0 & & 0 & & 1 & 3 \\
\hline 1739 & 0 & & 0 & & 1 & 3 \\
\hline 1744 & 1 & 2 & 0 & & 0 & \\
\hline 1745 & 1 & 2 & 0 & & 0 & \\
\hline 1747 & 1 & 2 & 0 & & 0 & \\
\hline
\end{tabular}


Table IV. (continued).

\begin{tabular}{|c|c|c|c|c|c|c|}
\hline year & $\begin{array}{c}\text { Etna } \\
\text { eruptions }\end{array}$ & VEI & $\begin{array}{l}\text { Stromboli } \\
\text { eruptions }\end{array}$ & VEI & $\begin{array}{l}\text { Vulcano } \\
\text { eruptions }\end{array}$ & VEI \\
\hline 1748 & 1 & 2 & 0 & & 0 & \\
\hline 1749 & 1 & 2 & 0 & & 0 & \\
\hline 1752 & 1 & 2 & 0 & & 0 & \\
\hline 1753 & 1 & 2 & 0 & & 0 & \\
\hline 1754 & 1 & 2 & 0 & & 0 & \\
\hline 1755 & 1 & 2 & 0 & & 0 & \\
\hline 1756 & 1 & 2 & 0 & & 0 & \\
\hline 1757 & 1 & 2 & 0 & & 0 & \\
\hline 1758 & 1 & 2 & 0 & & 0 & \\
\hline 1759 & 1 & 2 & 0 & & 0 & \\
\hline 1763 & 1 & 3 & 0 & & 0 & \\
\hline 1766 & 1 & 2 & 0 & & 0 & \\
\hline 1768 & 0 & & 1 & 2 & 0 & \\
\hline 1770 & 0 & & 1 & 2 & 0 & \\
\hline 1771 & 0 & & 0 & & 1 & 3 \\
\hline 1778 & 0 & & 1 & 2 & 0 & \\
\hline 1780 & 1 & 2 & 0 & & 1 & 2 \\
\hline 1781 & 1 & 2 & 0 & & 0 & \\
\hline 1786 & 0 & & 0 & & 1 & 3 \\
\hline 1787 & 1 & 4 & 0 & & 0 & \\
\hline 1791 & 1 & 2 & 0 & & 0 & \\
\hline 1792 & 1 & 3 & 0 & & 0 & \\
\hline 1793 & 1 & 3 & 0 & & 0 & \\
\hline 1797 & 1 & 2 & 0 & & 0 & \\
\hline 1798 & 1 & 2 & 0 & & 0 & \\
\hline 1799 & 1 & 2 & 0 & & 0 & \\
\hline 1800 & 1 & 2 & 0 & & 0 & \\
\hline 1801 & 1 & 2 & 0 & & 0 & \\
\hline 1802 & 1 & 2 & 0 & & 0 & \\
\hline 1803 & 1 & 2 & 0 & & 0 & \\
\hline 1804 & 1 & 2 & 0 & & 0 & \\
\hline 1805 & 1 & 2 & 0 & & 0 & \\
\hline 1806 & 1 & 2 & 0 & & 0 & \\
\hline 1807 & 1 & 2 & 0 & & 0 & \\
\hline 1808 & 1 & 2 & 0 & & 0 & \\
\hline 1809 & 1 & 2 & 0 & & 0 & \\
\hline
\end{tabular}


Table IV.(continued).

\begin{tabular}{|c|c|c|c|c|c|c|}
\hline year & $\begin{array}{c}\text { Etna } \\
\text { eruptions }\end{array}$ & VEI & $\begin{array}{l}\text { Stromboli } \\
\text { eruptions }\end{array}$ & VEI & $\begin{array}{l}\text { Vulcano } \\
\text { eruptions }\end{array}$ & VEI \\
\hline 1811 & 1 & 2 & 0 & & 0 & \\
\hline 1812 & 1 & 2 & 0 & & 0 & \\
\hline 1819 & 1 & 3 & 0 & & 0 & \\
\hline 1822 & 0 & & 1 & 3 & 1 & 2 \\
\hline 1823 & 0 & & 0 & & 1 & 2 \\
\hline 1828 & 1 & 2 & 0 & & 0 & \\
\hline 1829 & 1 & 2 & 0 & & 0 & \\
\hline 1830 & 1 & 2 & 0 & & 0 & \\
\hline 1831 & 1 & 2 & 0 & & 0 & \\
\hline 1832 & 1 & 2 & 0 & & 0 & \\
\hline 1833 & 1 & 2 & 1 & 2 & 0 & \\
\hline 1838 & 1 & 2 & 0 & & 0 & \\
\hline 1839 & 1 & 2 & 0 & & 0 & \\
\hline 1842 & 1 & 2 & 0 & & 0 & \\
\hline 1843 & 1 & 2 & 0 & & 0 & \\
\hline 1850 & 0 & & 1 & 2 & 0 & \\
\hline 1852 & 1 & 2 & 0 & & 0 & \\
\hline 1853 & 1 & 2 & 0 & & 0 & \\
\hline 1855 & 0 & & 1 & 2 & 0 & \\
\hline 1856 & 0 & & 1 & 2 & 0 & \\
\hline 1863 & 1 & 2 & 0 & & 0 & \\
\hline 1864 & 1 & 2 & 0 & & 0 & \\
\hline 1865 & 1 & 2 & 1 & 2 & 0 & \\
\hline 1868 & 1 & 3 & 0 & & 0 & \\
\hline 1873 & 0 & & 0 & & 1 & 3 \\
\hline 1874 & 1 & 2 & 1 & 2 & 1 & 3 \\
\hline 1875 & 0 & & 0 & & 1 & 3 \\
\hline 1876 & 0 & & 0 & & 1 & 3 \\
\hline 1877 & 0 & & 0 & & 1 & 3 \\
\hline 1878 & 1 & 2 & 0 & & 1 & 3 \\
\hline 1879 & 1 & 3 & 1 & 2 & 1 & 3 \\
\hline 1880 & 1 & 2 & 0 & & 0 & \\
\hline 1881 & 1 & 2 & 1 & 2 & 0 & \\
\hline 1882 & 1 & 2 & 1 & 3 & 0 & \\
\hline 1883 & 1 & 2 & 0 & & 0 & \\
\hline 1885 & 0 & & 1 & 2 & 0 & \\
\hline
\end{tabular}


Table IV. (continued).

\begin{tabular}{|c|c|c|c|c|c|c|}
\hline year & $\begin{array}{c}\text { Etna } \\
\text { eruptions }\end{array}$ & VEI & $\begin{array}{l}\text { Stromboli } \\
\text { eruptions }\end{array}$ & VEI & $\begin{array}{l}\text { Vulcano } \\
\text { eruptions }\end{array}$ & VEI \\
\hline 1886 & 1 & 3 & 0 & & 1 & 3 \\
\hline 1888 & 0 & & 1 & 2 & 1 & 3 \\
\hline 1889 & 0 & & 1 & 2 & 1 & 3 \\
\hline 1890 & 0 & & 0 & & 1 & 3 \\
\hline 1891 & 0 & & 1 & 3 & 0 & \\
\hline 1892 & 1 & 2 & 1 & 2 & 0 & \\
\hline 1893 & 1 & 2 & 1 & 2 & 0 & \\
\hline 1894 & 1 & 2 & 0 & & 0 & \\
\hline 1895 & 1 & 2 & 1 & 2 & 0 & \\
\hline 1896 & 1 & 2 & 1 & 2 & 0 & \\
\hline 1897 & 1 & 2 & 1 & 2 & 0 & \\
\hline 1898 & 1 & 2 & 1 & 2 & 0 & \\
\hline 1899 & 1 & 3 & 0 & & 0 & \\
\hline 1900 & 0 & & 1 & 2 & 0 & \\
\hline 1903 & 0 & & 1 & 2 & 0 & \\
\hline 1905 & 0 & & 1 & 2 & 0 & \\
\hline 1906 & 0 & & 1 & 2 & 0 & \\
\hline 1907 & 0 & & 1 & 3 & 0 & \\
\hline 1908 & 1 & 2 & 0 & & 0 & \\
\hline 1910 & 1 & 2 & 0 & & 0 & \\
\hline 1911 & 1 & 2 & 0 & & 0 & \\
\hline 1912 & 1 & 3 & 1 & 2 & 0 & \\
\hline 1913 & 1 & 2 & 0 & & 0 & \\
\hline 1914 & 1 & 2 & 0 & & 0 & \\
\hline 1915 & 1 & 2 & 1 & 2 & 0 & \\
\hline 1916 & 1 & 2 & 1 & 2 & 0 & \\
\hline 1917 & 1 & 2 & 0 & & 0 & \\
\hline 1919 & 1 & 2 & 1 & 3 & 0 & \\
\hline 1920 & 1 & 2 & 0 & & 0 & \\
\hline 1921 & 1 & 2 & 1 & 2 & 0 & \\
\hline 1922 & 1 & 2 & 0 & & 0 & \\
\hline 1923 & 1 & 2 & 0 & & 0 & \\
\hline 1930 & 0 & & 1 & 3 & 0 & \\
\hline 1931 & 1 & 2 & 0 & & 0 & \\
\hline 1932 & 1 & 2 & 0 & & 0 & \\
\hline 1933 & 1 & 2 & 0 & & 0 & \\
\hline
\end{tabular}


Table IV.(continued).

\begin{tabular}{|c|c|c|c|c|c|c|}
\hline year & $\begin{array}{c}\text { Etna } \\
\text { eruptions }\end{array}$ & VEI & $\begin{array}{l}\text { Stromboli } \\
\text { eruptions }\end{array}$ & VEI & $\begin{array}{l}\text { Vulcano } \\
\text { eruptions }\end{array}$ & VEI \\
\hline 1934 & 0 & & 1 & 2 & 0 & \\
\hline 1935 & 1 & 2 & 0 & & 0 & \\
\hline 1936 & 1 & 2 & 1 & 2 & 0 & \\
\hline 1937 & 1 & 2 & 1 & 2 & 0 & \\
\hline 1938 & 1 & 2 & 1 & 2 & 0 & \\
\hline 1939 & 1 & 2 & 1 & 2 & 0 & \\
\hline 1940 & 1 & 3 & 0 & & 0 & \\
\hline 1941 & 1 & 3 & 1 & 2 & 0 & \\
\hline 1942 & 1 & 3 & 0 & & 0 & \\
\hline 1943 & 0 & & 1 & 2 & 0 & \\
\hline 1944 & 0 & & 1 & 2 & 0 & \\
\hline 1947 & 1 & 2 & 0 & & 0 & \\
\hline 1949 & 1 & 2 & 1 & 2 & 0 & \\
\hline 1950 & 1 & 2 & 1 & 2 & 0 & \\
\hline 1951 & 1 & 2 & 1 & 2 & 0 & \\
\hline 1952 & 0 & & 1 & 2 & 0 & \\
\hline 1953 & 1 & 2 & 0 & & 0 & \\
\hline 1954 & 0 & & 1 & 2 & 0 & \\
\hline 1955 & 1 & 2 & 1 & 2 & 0 & \\
\hline 1956 & 1 & 2 & 0 & & 0 & \\
\hline 1957 & 1 & 2 & 0 & & 0 & \\
\hline 1958 & 1 & 2 & 0 & & 0 & \\
\hline 1959 & 1 & 3 & 1 & 2 & 0 & \\
\hline 1960 & 1 & 3 & 0 & & 0 & \\
\hline 1961 & 1 & 3 & 0 & & 0 & \\
\hline 1962 & 1 & 3 & 0 & & 0 & \\
\hline 1963 & 1 & 3 & 0 & & 0 & \\
\hline 1964 & 1 & 3 & 0 & & 0 & \\
\hline 1966 & 1 & 2 & 1 & 2 & 0 & \\
\hline 1967 & 1 & 2 & 0 & & 0 & \\
\hline 1968 & 1 & 2 & 0 & & 0 & \\
\hline 1969 & 1 & 2 & 0 & & 0 & \\
\hline 1970 & 1 & 2 & 0 & & 0 & \\
\hline 1971 & 1 & 2 & 1 & 2 & 0 & \\
\hline 1972 & 1 & 2 & 0 & & 0 & \\
\hline 1973 & 1 & 2 & 0 & & 0 & \\
\hline
\end{tabular}


Table IV. (continued).

\begin{tabular}{|c|c|c|c|c|c|c|}
\hline year & $\begin{array}{c}\text { Etna } \\
\text { eruptions }\end{array}$ & VEI & $\begin{array}{l}\text { Stromboli } \\
\text { eruptions }\end{array}$ & VEI & $\begin{array}{l}\text { Vulcano } \\
\text { eruptions }\end{array}$ & VEI \\
\hline 1974 & 1 & 2 & 0 & & 0 & \\
\hline 1975 & 1 & 2 & 0 & & 0 & \\
\hline 1976 & 1 & 2 & 0 & & 0 & \\
\hline 1977 & 1 & 2 & 0 & & 0 & \\
\hline 1978 & 1 & 2 & 0 & & 0 & \\
\hline 1979 & 1 & 2 & 0 & & 0 & \\
\hline 1980 & 1 & 3 & 0 & & 0 & \\
\hline 1981 & 1 & 2 & 0 & & 0 & \\
\hline 1982 & 1 & 2 & 0 & & 0 & \\
\hline 1983 & 1 & 2 & 0 & & 0 & \\
\hline 1984 & 1 & 2 & 0 & & 0 & \\
\hline 1985 & 1 & 2 & 1 & 2 & 0 & \\
\hline 1986 & 1 & 2 & 1 & 2 & 0 & \\
\hline 1987 & 1 & 2 & 0 & & 0 & \\
\hline 1988 & 1 & 2 & 0 & & 0 & \\
\hline 1989 & 1 & 2 & 0 & & 0 & \\
\hline 1990 & 1 & 2 & 0 & & 0 & \\
\hline 1991 & 1 & 2 & 0 & & 0 & \\
\hline 1992 & 1 & 2 & 0 & & 0 & \\
\hline 1993 & 1 & 2 & 0 & & 0 & \\
\hline
\end{tabular}

Table V. Monthly totals of seismic moment $\left(\mathrm{TM}_{0}\right)$ for the Etnean and Aeolian areas, from January 1983 to December 2007 (i.e. 300 months). Seismic moments are computed from earthquake magnitudes provided in the INGV catalogue of earthquakes available online at www.ingv.it (Bollettino Sismico Italiano, Istituto Nazionale di Geofisica e Vulcanologia). Focal depth for the considered earthquakes is less than $30 \mathrm{~km}$. 0 is where no records are available.

\begin{tabular}{rccc}
\hline \hline year & month & TM $\boldsymbol{M}_{\mathbf{0}}$ Etna $($ dyn $\mathbf{c m})$ & TM \\
\hline 1983 & Jan. & 0 & 0 \\
1983 & Feb. & 0 & 0 \\
1983 & Mar. & $8.61157 \mathrm{E}+21$ & 0 \\
1983 & Apr. & 0 & 0 \\
1983 & May & 0 & 0 \\
1983 & Jun. & $3.1335 \mathrm{E}+21$ & 0 \\
1983 & Jul. & 0 & 0 \\
1983 & Aug. & 0 & 0 \\
\hline
\end{tabular}


Table V. (continued).

\begin{tabular}{|c|c|c|c|}
\hline year & month & $T M_{0}$ Etna $($ dyn cm) & $T M_{0}$ Aeolian(dyn cm) \\
\hline 1983 & Sep. & 0 & $2.21309 \mathrm{E}+21$ \\
\hline 1983 & Oct. & $3.68198 \mathrm{E}+21$ & $2.21309 \mathrm{E}+21$ \\
\hline 1983 & Nov. & 0 & 0 \\
\hline 1983 & Dec. & 0 & 0 \\
\hline 1984 & Jan. & 0 & $7.53147 \mathrm{E}+20$ \\
\hline 1984 & Feb. & 0 & 0 \\
\hline 1984 & Mar. & 0 & 0 \\
\hline 1984 & Apr. & 0 & 0 \\
\hline 1984 & May & 0 & $2.78612 \mathrm{E}+20$ \\
\hline 1984 & Jun. & $2.72893 \mathrm{E}+21$ & $3.9355 \mathrm{E}+20$ \\
\hline 1984 & Jul. & $1.50272 \mathrm{E}+21$ & 0 \\
\hline 1984 & Aug. & $5.81437 \mathrm{E}+21$ & $7.85236 \mathrm{E}+20$ \\
\hline 1984 & Sep. & $1.10917 \mathrm{E}+21$ & $1.56675 \mathrm{E}+21$ \\
\hline 1984 & Oct. & $5.52259 \mathrm{E}+21$ & 0 \\
\hline 1984 & Nov. & $3.67231 \mathrm{E}+21$ & $5.55904 \mathrm{E}+20$ \\
\hline 1984 & Dec. & 0 & 0 \\
\hline 1985 & Jan. & 0 & 0 \\
\hline 1985 & Feb. & $1.1714 \mathrm{E}+21$ & 0 \\
\hline 1985 & Mar. & $4.33707 \mathrm{E}+21$ & 0 \\
\hline 1985 & Apr. & $1.343 \mathrm{E}+21$ & 0 \\
\hline 1985 & May & 0 & 0 \\
\hline 1985 & Jun. & $3.9355 E+20$ & 0 \\
\hline 1985 & Jul. & 0 & 0 \\
\hline 1985 & Aug. & $5.55904 \mathrm{E}+20$ & 0 \\
\hline 1985 & Sep. & $3.12608 \mathrm{E}+21$ & $3.9355 \mathrm{E}+20$ \\
\hline 1985 & Oct. & $2.21835 \mathrm{E}+21$ & 0 \\
\hline 1985 & Nov. & $2.08488 \mathrm{E}+21$ & 0 \\
\hline 1985 & Dec. & $1.68442 \mathrm{E}+22$ & $9.24872 \mathrm{E}+20$ \\
\hline 1986 & Jan. & $6.15005 E+21$ & 0 \\
\hline 1986 & Feb. & $1.97242 \mathrm{E}+20$ & 0 \\
\hline 1986 & Mar. & $1.13198 \mathrm{E}+21$ & $3.9355 \mathrm{E}+20$ \\
\hline 1986 & Apr. & $2.78612 \mathrm{E}+20$ & 0 \\
\hline 1986 & May & $7.10694 \mathrm{E}+21$ & $2.21309 \mathrm{E}+21$ \\
\hline 1986 & Jun. & $3.77467 \mathrm{E}+20$ & $1.97242 \mathrm{E}+20$ \\
\hline 1986 & Jul. & $3.26358 \mathrm{E}+21$ & 0 \\
\hline
\end{tabular}


Table V. (continued).

\begin{tabular}{|c|c|c|c|}
\hline year & month & $T M_{0}$ Etna $($ dyn $\mathrm{cm})$ & TM $M_{0}$ Aeolian(dyn cm) \\
\hline 1986 & Aug. & $4.75854 \mathrm{E}+20$ & 0 \\
\hline 1986 & Sep. & $1.35443 \mathrm{E}+21$ & $1.97242 \mathrm{E}+20$ \\
\hline 1986 & Oct. & $1.96846 \mathrm{E}+22$ & $7.94256 \mathrm{E}+21$ \\
\hline 1986 & Nov. & $2.23793 \mathrm{E}+21$ & 0 \\
\hline 1986 & Dec. & $2.48313 \mathrm{E}+19$ & 0 \\
\hline 1987 & Jan. & $2.38492 \mathrm{E}+20$ & 0 \\
\hline 1987 & Feb. & $4.05612 \mathrm{E}+21$ & $4.9545 \mathrm{E}+19$ \\
\hline 1987 & Mar. & 0 & 0 \\
\hline 1987 & Apr. & 0 & 0 \\
\hline 1987 & May & $6.76753 \mathrm{E}+20$ & $9.88553 \mathrm{E}+19$ \\
\hline 1987 & Jun. & $9.88553 \mathrm{E}+19$ & 0 \\
\hline 1987 & Jul. & $4.18381 \mathrm{E}+20$ & 0 \\
\hline 1987 & Aug. & $1.12675 \mathrm{E}+21$ & $2.96191 \mathrm{E}+20$ \\
\hline 1987 & Sep. & $8.84091 \mathrm{E}+20$ & $5.55904 \mathrm{E}+20$ \\
\hline 1987 & Oct. & $6.99842 \mathrm{E}+19$ & $9.88553 \mathrm{E}+19$ \\
\hline 1987 & Nov. & $3.28157 \mathrm{E}+20$ & 0 \\
\hline 1987 & Dec. & $4.9545 \mathrm{E}+19$ & $2.67695 \mathrm{E}+20$ \\
\hline 1988 & Jan. & $3.13687 \mathrm{E}+20$ & 0 \\
\hline 1988 & Feb. & $3.50752 E+19$ & 0 \\
\hline 1988 & Mar. & 0 & $5.18173 \mathrm{E}+21$ \\
\hline 1988 & Apr. & $1.40989 \mathrm{E}+21$ & $2.36029 \mathrm{E}+21$ \\
\hline 1988 & May & 0 & $3.23671 \mathrm{E}+21$ \\
\hline 1988 & Jun. & $1.27126 \mathrm{E}+21$ & $3.84779 \mathrm{E}+21$ \\
\hline 1988 & Jul. & $2.43216 \mathrm{E}+20$ & 0 \\
\hline 1988 & Aug. & $2.70863 \mathrm{E}+21$ & $4.7803 \mathrm{E}+21$ \\
\hline 1988 & Sep. & $1.75792 \mathrm{E}+19$ & $4.9545 \mathrm{E}+19$ \\
\hline 1988 & Oct. & $6.99842 \mathrm{E}+19$ & $1.44361 \mathrm{E}+20$ \\
\hline 1988 & Nov. & $6.27679 \mathrm{E}+20$ & $6.99842 \mathrm{E}+19$ \\
\hline 1988 & Dec. & $7.96041 \mathrm{E}+20$ & $9.88553 \mathrm{E}+19$ \\
\hline 1989 & Jan. & $7.56663 \mathrm{E}+20$ & $3.50752 \mathrm{E}+19$ \\
\hline 1989 & Feb. & $3.44341 \mathrm{E}+21$ & $5.58018 \mathrm{E}+20$ \\
\hline 1989 & Mar. & $3.34351 \mathrm{E}+20$ & $1.6884 \mathrm{E}+20$ \\
\hline 1989 & Apr. & $2.18737 \mathrm{E}+20$ & $1.05059 \mathrm{E}+20$ \\
\hline 1989 & May & $6.41844 \mathrm{E}+20$ & $2.18385 \mathrm{E}+20$ \\
\hline 1989 & Jun. & $2.37224 \mathrm{E}+21$ & $1.39637 \mathrm{E}+20$ \\
\hline
\end{tabular}


Table V. (continued).

\begin{tabular}{|c|c|c|c|}
\hline year & month & $T M_{0}$ Etna $($ dyn $\mathrm{cm})$ & TM $M_{0}$ Aeolian $($ dyn $\mathrm{cm})$ \\
\hline 1989 & Jul. & $3.64362 \mathrm{E}+21$ & $1.41525 \mathrm{E}+21$ \\
\hline 1989 & Aug. & $1.28044 \mathrm{E}+22$ & $2.44696 \mathrm{E}+20$ \\
\hline 1989 & Sep. & $5.81645 \mathrm{E}+21$ & $6.72162 \mathrm{E}+20$ \\
\hline 1989 & Oct. & $7.8372 \mathrm{E}+21$ & $2.83997 \mathrm{E}+20$ \\
\hline 1989 & Nov. & $1.56675 \mathrm{E}+21$ & $6.71243 \mathrm{E}+19$ \\
\hline 1989 & Dec. & $6.99842 \mathrm{E}+19$ & $6.99842 \mathrm{E}+19$ \\
\hline 1990 & Jan. & $3.2323 \mathrm{E}+20$ & 0 \\
\hline 1990 & Feb. & $2.3495 \mathrm{E}+20$ & $1.19391 \mathrm{E}+21$ \\
\hline 1990 & Mar. & $1.07113 \mathrm{E}+22$ & $2.52683 \mathrm{E}+21$ \\
\hline 1990 & Apr. & $5.3811 \mathrm{E}+20$ & $3.34819 \mathrm{E}+20$ \\
\hline 1990 & May & $1.63593 \mathrm{E}+21$ & $4.9545 \mathrm{E}+19$ \\
\hline 1990 & Jun. & $2.78612 \mathrm{E}+20$ & 0 \\
\hline 1990 & Jul. & $6.50577 \mathrm{E}+20$ & 0 \\
\hline 1990 & Aug. & $1.43476 \mathrm{E}+21$ & $2.48313 \mathrm{E}+19$ \\
\hline 1990 & Sep. & $3.21509 \mathrm{E}+21$ & $1.63863 \mathrm{E}+21$ \\
\hline 1990 & Oct. & $1.69074 \mathrm{E}+20$ & $4.18381 \mathrm{E}+20$ \\
\hline 1990 & Nov. & $7.01504 \mathrm{E}+19$ & $1.05059 \mathrm{E}+20$ \\
\hline 1990 & Dec. & $4.36437 \mathrm{E}+20$ & 0 \\
\hline 1991 & Jan. & $2.78516 \mathrm{E}+21$ & $1.69192 \mathrm{E}+20$ \\
\hline 1991 & Feb. & $3.80765 \mathrm{E}+20$ & 0 \\
\hline 1991 & Mar. & $1.39637 \mathrm{E}+20$ & 0 \\
\hline 1991 & Apr. & $1.58196 \mathrm{E}+21$ & $7.03374 \mathrm{E}+20$ \\
\hline 1991 & May & $2.62578 \mathrm{E}+21$ & 0 \\
\hline 1991 & Jun. & $5.05719 \mathrm{E}+20$ & 0 \\
\hline 1991 & Jul. & $4.21968 \mathrm{E}+20$ & 4.9545E+19 \\
\hline 1991 & Aug. & $3.28049 \mathrm{E}+20$ & $5.99065 \mathrm{E}+19$ \\
\hline 1991 & Sep. & $3.6788 \mathrm{E}+21$ & $2.92271 \mathrm{E}+21$ \\
\hline 1991 & Oct. & $8.92828 \mathrm{E}+20$ & 0 \\
\hline 1991 & Nov. & $2.92217 \mathrm{E}+20$ & $6.99842 \mathrm{E}+19$ \\
\hline 1991 & Dec. & $7.58078 \mathrm{E}+20$ & 0 \\
\hline 1992 & Jan. & $1.2995 \mathrm{E}+20$ & $6.99842 \mathrm{E}+19$ \\
\hline 1992 & Feb. & $6.82522 \mathrm{E}+20$ & $6.99842 \mathrm{E}+19$ \\
\hline 1992 & Mar. & $6.99842 \mathrm{E}+19$ & $1.25758 \mathrm{E}+21$ \\
\hline 1992 & Apr. & $1.75792 \mathrm{E}+19$ & $9.48155 \mathrm{E}+19$ \\
\hline 1992 & May & $3.45643 \mathrm{E}+20$ & $1.40218 \mathrm{E}+20$ \\
\hline
\end{tabular}


Table V. (continued).

\begin{tabular}{|c|c|c|c|}
\hline year & month & $T M_{0}$ Etna $($ dyn $\mathrm{cm})$ & TM $M_{0}$ Aeolian(dyn cm) \\
\hline 1992 & Jun. & $1.19813 \mathrm{E}+20$ & $1.41266 \mathrm{E}+20$ \\
\hline 1992 & Jul. & $1.97242 \mathrm{E}+20$ & $4.28625 \mathrm{E}+20$ \\
\hline 1992 & Aug. & $9.88553 \mathrm{E}+19$ & $1.39637 \mathrm{E}+20$ \\
\hline 1992 & Sep. & $1.57216 \mathrm{E}+20$ & $1.89513 \mathrm{E}+20$ \\
\hline 1992 & Oct. & 0 & 0 \\
\hline 1992 & Nov. & 0 & $4.63534 \mathrm{E}+20$ \\
\hline 1992 & Dec. & 0 & $3.9355 \mathrm{E}+20$ \\
\hline 1993 & Jan. & 0 & $2.58995 \mathrm{E}+21$ \\
\hline 1993 & Feb. & $6.99842 \mathrm{E}+19$ & $4.9545 \mathrm{E}+19$ \\
\hline 1993 & Mar. & $4.9545 \mathrm{E}+19$ & $2.28308 \mathrm{E}+21$ \\
\hline 1993 & Apr. & $1.19529 \mathrm{E}+20$ & $1.97242 \mathrm{E}+20$ \\
\hline 1993 & May & $1.48635 \mathrm{E}+20$ & $3.78461 \mathrm{E}+20$ \\
\hline 1993 & Jun. & $4.98609 \mathrm{E}+20$ & 0 \\
\hline 1993 & Jul. & $9.50481 \mathrm{E}+20$ & $1.25316 \mathrm{E}+21$ \\
\hline 1993 & Aug. & $3.50456 \mathrm{E}+21$ & $5.90792 \mathrm{E}+20$ \\
\hline 1993 & Sep. & $4.96627 \mathrm{E}+19$ & $1.20803 \mathrm{E}+21$ \\
\hline 1993 & Oct. & $4.9545 \mathrm{E}+19$ & $5.92524 \mathrm{E}+20$ \\
\hline 1993 & Nov. & 0 & 0 \\
\hline 1993 & Dec. & $2.79274 \mathrm{E}+20$ & 0 \\
\hline 1994 & Jan. & 0 & 0 \\
\hline 1994 & Feb. & $1.8968 \mathrm{E}+20$ & $8.46202 \mathrm{E}+19$ \\
\hline 1994 & Mar. & $4.9545 \mathrm{E}+19$ & $5.83666 \mathrm{E}+20$ \\
\hline 1994 & Apr. & $9.88553 \mathrm{E}+19$ & $2.48313 \mathrm{E}+19$ \\
\hline 1994 & May & $1.42953 \mathrm{E}+21$ & $5.05719 \mathrm{E}+20$ \\
\hline 1994 & Jun. & $8.47035 \mathrm{E}+19$ & $6.99842 \mathrm{E}+19$ \\
\hline 1994 & Jul. & $5.99065 \mathrm{E}+19$ & $4.70778 \mathrm{E}+21$ \\
\hline 1994 & Aug. & $6.99842 \mathrm{E}+19$ & 0 \\
\hline 1994 & Sep. & $2.53066 \mathrm{E}+21$ & $1.44361 \mathrm{E}+20$ \\
\hline 1994 & Oct. & $3.89057 \mathrm{E}+20$ & $2.94814 \mathrm{E}+21$ \\
\hline 1994 & Nov. & $2.80103 \mathrm{E}+20$ & $5.59765 \mathrm{E}+21$ \\
\hline 1994 & Dec. & $7.81624 \mathrm{E}+20$ & $5.92299 \mathrm{E}+20$ \\
\hline 1995 & Jan. & $1.75792 \mathrm{E}+19$ & $9.88553 E+19$ \\
\hline 1995 & Feb. & $6.44712 \mathrm{E}+21$ & $2.38492 \mathrm{E}+20$ \\
\hline 1995 & Mar. & 0 & 0 \\
\hline 1995 & Apr. & 0 & $3.58021 \mathrm{E}+20$ \\
\hline
\end{tabular}


Table V. (continued).

\begin{tabular}{|c|c|c|c|}
\hline year & month & $T M_{0}$ Etna $($ dyn cm) & TM $M_{0}$ Aeolian $(\mathrm{dyn} \mathrm{cm})$ \\
\hline 1995 & May & $3.19881 \mathrm{E}+20$ & 0 \\
\hline 1995 & Jun. & $9.48155 \mathrm{E}+19$ & 0 \\
\hline 1995 & Jul. & $1.39637 \mathrm{E}+20$ & $5.6121 \mathrm{E}+22$ \\
\hline 1995 & Aug. & $8.22263 \mathrm{E}+20$ & $1.25151 \mathrm{E}+22$ \\
\hline 1995 & Sep. & $2.48313 \mathrm{E}+19$ & $5.33685 \mathrm{E}+20$ \\
\hline 1995 & Oct. & $2.38727 \mathrm{E}+20$ & $9.51834 \mathrm{E}+20$ \\
\hline 1995 & Nov. & $9.88553 \mathrm{E}+19$ & $4.9545 \mathrm{E}+19$ \\
\hline 1995 & Dec. & $7.87947 \mathrm{E}+19$ & 0 \\
\hline 1996 & Jan. & $9.909 \mathrm{E}+19$ & $4.35734 \mathrm{E}+20$ \\
\hline 1996 & Feb. & $2.64367 \mathrm{E}+20$ & $1.97242 \mathrm{E}+20$ \\
\hline 1996 & Mar. & $1.23687 \mathrm{E}+20$ & $1.39637 \mathrm{E}+20$ \\
\hline 1996 & Apr. & $1.34014 \mathrm{E}+20$ & 0 \\
\hline 1996 & May & 0 & $5.7471 \mathrm{E}+20$ \\
\hline 1996 & Jun. & $9.48155 E+19$ & $1.38779 \mathrm{E}+21$ \\
\hline 1996 & Jul. & $6.99842 \mathrm{E}+19$ & $8.24451 \mathrm{E}+20$ \\
\hline 1996 & Aug. & $1.2659 \mathrm{E}+21$ & $1.97242 \mathrm{E}+20$ \\
\hline 1996 & Sep. & $6.27505 \mathrm{E}+20$ & $6.25888 \mathrm{E}+20$ \\
\hline 1996 & Oct. & 0 & 0 \\
\hline 1996 & Nov. & $5.23153 \mathrm{E}+20$ & 0 \\
\hline 1996 & Dec. & $2.12613 \mathrm{E}+21$ & 0 \\
\hline 1997 & Jan. & $3.36345 \mathrm{E}+21$ & $3.9355 \mathrm{E}+20$ \\
\hline 1997 & Feb. & $4.74944 \mathrm{E}+21$ & $8.45102 \mathrm{E}+20$ \\
\hline 1997 & Mar. & $4.92758 \mathrm{E}+20$ & $1.25972 \mathrm{E}+21$ \\
\hline 1997 & Apr. & $1.25142 \mathrm{E}+21$ & 0 \\
\hline 1997 & May & $7.16238 \mathrm{E}+20$ & $1.77135 \mathrm{E}+21$ \\
\hline 1997 & Jun. & $7.73346 \mathrm{E}+20$ & $9.88553 \mathrm{E}+19$ \\
\hline 1997 & Jul. & $7.36677 \mathrm{E}+20$ & $1.05059 \mathrm{E}+20$ \\
\hline 1997 & Aug. & $4.40469 \mathrm{E}+21$ & 0 \\
\hline 1997 & Sep. & $2.60291 \mathrm{E}+21$ & $2.42808 \mathrm{E}+21$ \\
\hline 1997 & Oct. & $3.8698 \mathrm{E}+21$ & $1.75792 \mathrm{E}+19$ \\
\hline 1997 & Nov. & $9.91181 \mathrm{E}+20$ & $2.3411 \mathrm{E}+21$ \\
\hline 1997 & Dec. & $2.50586 \mathrm{E}+22$ & $3.48596 \mathrm{E}+20$ \\
\hline 1998 & Jan. & $2.10765 \mathrm{E}+22$ & 0 \\
\hline 1998 & Feb. & $3.9807 \mathrm{E}+20$ & $8.70253 \mathrm{E}+20$ \\
\hline 1998 & Mar. & $4.77556 \mathrm{E}+21$ & 0 \\
\hline
\end{tabular}


Table V. (continued).

\begin{tabular}{|c|c|c|c|}
\hline year & month & $T M_{0}$ Etna $($ dyn $\mathrm{cm})$ & TM $M_{0}$ Aeolian(dyn cm) \\
\hline 1998 & Apr. & $2.34619 \mathrm{E}+20$ & $9.12398 \mathrm{E}+19$ \\
\hline 1998 & May & $2.2054 \mathrm{E}+21$ & 0 \\
\hline 1998 & Jun. & $6.07607 \mathrm{E}+20$ & $6.79591 \mathrm{E}+20$ \\
\hline 1998 & Jul. & $2.38492 \mathrm{E}+20$ & $4.43095 \mathrm{E}+20$ \\
\hline 1998 & Aug. & $1.0944 \mathrm{E}+21$ & $1.39968 \mathrm{E}+20$ \\
\hline 1998 & Sep. & $6.75551 \mathrm{E}+20$ & $5.7471 \mathrm{E}+20$ \\
\hline 1998 & Oct. & $4.30705 \mathrm{E}+20$ & 0 \\
\hline 1998 & Nov. & $1.44361 \mathrm{E}+20$ & 0 \\
\hline 1998 & Dec. & $2.34753 \mathrm{E}+21$ & 0 \\
\hline 1999 & Jan. & $6.46722 \mathrm{E}+20$ & $4.18249 \mathrm{E}+20$ \\
\hline 1999 & Feb. & $2.18658 \mathrm{E}+21$ & $6.53665 \mathrm{E}+20$ \\
\hline 1999 & Mar. & $2.01256 \mathrm{E}+21$ & $2.59166 \mathrm{E}+20$ \\
\hline 1999 & Apr. & $5.63324 \mathrm{E}+20$ & $1.97242 \mathrm{E}+20$ \\
\hline 1999 & May & $4.01157 \mathrm{E}+20$ & $1.6884 \mathrm{E}+20$ \\
\hline 1999 & Jun. & $9.30161 \mathrm{E}+20$ & 0 \\
\hline 1999 & Jul. & $1.73232 \mathrm{E}+20$ & 0 \\
\hline 1999 & Aug. & $3.52873 \mathrm{E}+21$ & $3.9355 \mathrm{E}+20$ \\
\hline 1999 & Sep. & $6.42838 \mathrm{E}+20$ & $1.74712 \mathrm{E}+20$ \\
\hline 1999 & Oct. & $1.55728 \mathrm{E}+21$ & $1.23687 \mathrm{E}+20$ \\
\hline 1999 & Nov. & $1.484 \mathrm{E}+20$ & 0 \\
\hline 1999 & Dec. & $4.18381 \mathrm{E}+20$ & $2.01055 \mathrm{E}+20$ \\
\hline 2000 & Jan. & $1.3795 \mathrm{E}+21$ & 0 \\
\hline 2000 & Feb. & $1.93858 \mathrm{E}+21$ & 0 \\
\hline 2000 & Mar. & $1.71442 \mathrm{E}+20$ & 0 \\
\hline 2000 & Apr. & $6.5476 \mathrm{E}+20$ & $2.54997 \mathrm{E}+21$ \\
\hline 2000 & May & $2.46787 \mathrm{E}+20$ & 0 \\
\hline 2000 & Jun. & 0 & $1.39637 \mathrm{E}+20$ \\
\hline 2000 & Jul. & $5.34921 \mathrm{E}+20$ & $4.28625 \mathrm{E}+20$ \\
\hline 2000 & Aug. & $2.92692 \mathrm{E}+20$ & 0 \\
\hline 2000 & Sep. & $9.89385 \mathrm{E}+20$ & $1.39637 \mathrm{E}+20$ \\
\hline 2000 & Oct. & $6.02056 \mathrm{E}+20$ & $6.99842 \mathrm{E}+19$ \\
\hline 2000 & Nov. & $1.68763 \mathrm{E}+22$ & 0 \\
\hline 2000 & Dec. & $1.29617 \mathrm{E}+21$ & 0 \\
\hline 2001 & Jan. & $2.26636 \mathrm{E}+21$ & 0 \\
\hline 2001 & Feb. & $5.35177 \mathrm{E}+20$ & $1.97242 \mathrm{E}+20$ \\
\hline
\end{tabular}


Table V. (continued).

\begin{tabular}{|c|c|c|c|}
\hline year & month & $T M_{0}$ Etna $($ dyn cm) & TM $M_{0}$ Aeolian $(\mathrm{dyn} \mathrm{cm})$ \\
\hline 2001 & Mar. & $7.57161 \mathrm{E}+20$ & 0 \\
\hline 2001 & Apr. & $5.69959 \mathrm{E}+21$ & 0 \\
\hline 2001 & May & $4.65916 \mathrm{E}+21$ & $1.75792 \mathrm{E}+19$ \\
\hline 2001 & Jun. & $3.43712 \mathrm{E}+20$ & $4.9545 \mathrm{E}+19$ \\
\hline 2001 & Jul. & $5.18235 \mathrm{E}+22$ & $1.14118 \mathrm{E}+22$ \\
\hline 2001 & Aug. & $1.68645 \mathrm{E}+21$ & $8.69404 \mathrm{E}+20$ \\
\hline 2001 & Sep. & $2.48313 \mathrm{E}+19$ & $8.5522 \mathrm{E}+20$ \\
\hline 2001 & Oct. & $5.96325 \mathrm{E}+21$ & $5.69665 \mathrm{E}+20$ \\
\hline 2001 & Nov. & 0 & $7.85236 \mathrm{E}+20$ \\
\hline 2001 & Dec. & $6.99842 \mathrm{E}+19$ & 0 \\
\hline 2002 & Jan. & $3.28157 \mathrm{E}+20$ & 0 \\
\hline 2002 & Feb. & $1.74712 \mathrm{E}+20$ & $1.39637 \mathrm{E}+20$ \\
\hline 2002 & Mar. & $3.28819 \mathrm{E}+20$ & $1.3422 \mathrm{E}+21$ \\
\hline 2002 & Apr. & $2.09621 \mathrm{E}+20$ & $9.86941 \mathrm{E}+21$ \\
\hline 2002 & May & $2.14345 \mathrm{E}+20$ & $2.78612 \mathrm{E}+20$ \\
\hline 2002 & Jun. & $1.89513 \mathrm{E}+20$ & $8.34516 \mathrm{E}+20$ \\
\hline 2002 & Jul. & $7.30429 \mathrm{E}+20$ & 0 \\
\hline 2002 & Aug. & $1.53444 \mathrm{E}+21$ & $1.39637 \mathrm{E}+20$ \\
\hline 2002 & Sep. & $2.46787 \mathrm{E}+20$ & $6.95541 \mathrm{E}+20$ \\
\hline 2002 & Oct. & $1.07678 \mathrm{E}+23$ & $1.19529 \mathrm{E}+20$ \\
\hline 2002 & Nov. & $5.43328 \mathrm{E}+21$ & 0 \\
\hline 2002 & Dec. & $2.25681 \mathrm{E}+21$ & $3.50752 \mathrm{E}+19$ \\
\hline 2003 & Jan. & $1.6884 \mathrm{E}+20$ & 0 \\
\hline 2003 & Feb. & $8.95013 \mathrm{E}+21$ & $2.78612 \mathrm{E}+20$ \\
\hline 2003 & Mar. & $2.09621 \mathrm{E}+20$ & $2.48313 \mathrm{E}+19$ \\
\hline 2003 & Apr. & 0 & 0 \\
\hline 2003 & May & $1.39968 \mathrm{E}+20$ & 0 \\
\hline 2003 & Jun. & $1.97242 \mathrm{E}+20$ & $5.75371 \mathrm{E}+20$ \\
\hline 2003 & Jul. & $1.95985 \mathrm{E}+21$ & 0 \\
\hline 2003 & Aug. & $2.78612 \mathrm{E}+20$ & 0 \\
\hline 2003 & Sep. & 0 & 0 \\
\hline 2003 & Oct. & $2.18385 \mathrm{E}+20$ & 0 \\
\hline 2003 & Nov. & 0 & $1.6884 \mathrm{E}+20$ \\
\hline 2003 & Dec. & $1.6884 \mathrm{E}+20$ & 0 \\
\hline 2004 & Jan. & $2.32317 \mathrm{E}+20$ & $1.6884 \mathrm{E}+20$ \\
\hline
\end{tabular}


Table V. (continued).

\begin{tabular}{|c|c|c|c|}
\hline year & month & $T M_{0}$ Etna $($ dyn $\mathrm{cm})$ & TM $M_{0}$ Aeolian(dyn cm) \\
\hline 2004 & Feb. & $7.65504 \mathrm{E}+20$ & 0 \\
\hline 2004 & Mar. & $8.92783 \mathrm{E}+20$ & $2.50113 \mathrm{E}+21$ \\
\hline 2004 & Apr. & 0 & $3.17562 \mathrm{E}+21$ \\
\hline 2004 & May & $7.57776 \mathrm{E}+20$ & $2.39508 \mathrm{E}+20$ \\
\hline 2004 & Jun. & $2.20707 \mathrm{E}+21$ & $6.99842 \mathrm{E}+19$ \\
\hline 2004 & Jul. & $1.39578 \mathrm{E}+21$ & $1.14425 \mathrm{E}+21$ \\
\hline 2004 & Aug. & $2.38824 \mathrm{E}+20$ & 0 \\
\hline 2004 & Sep. & $7.43764 \mathrm{E}+19$ & $5.99065 \mathrm{E}+19$ \\
\hline 2004 & Oct. & $3.50752 \mathrm{E}+19$ & $5.07524 \mathrm{E}+20$ \\
\hline 2004 & Nov. & $9.88553 \mathrm{E}+19$ & $8.46202 \mathrm{E}+19$ \\
\hline 2004 & Dec. & $6.99842 \mathrm{E}+19$ & 0 \\
\hline 2005 & Jan. & $1.97242 \mathrm{E}+20$ & $7.85236 \mathrm{E}+20$ \\
\hline 2005 & Feb. & $1.39637 \mathrm{E}+20$ & 0 \\
\hline 2005 & Mar. & 0 & 0 \\
\hline 2005 & Apr. & $4.97243 \mathrm{E}+20$ & $6.23735 \mathrm{E}+18$ \\
\hline 2005 & May & $9.48155 \mathrm{E}+19$ & $3.10687 \mathrm{E}+19$ \\
\hline 2005 & Jun. & $4.38857 \mathrm{E}+19$ & $1.79728 \mathrm{E}+19$ \\
\hline 2005 & Jul. & $3.44522 \mathrm{E}+21$ & $1.75792 \mathrm{E}+19$ \\
\hline 2005 & Aug. & $1.42534 \mathrm{E}+21$ & $4.24106 \mathrm{E}+19$ \\
\hline 2005 & Sep. & $3.50752 \mathrm{E}+19$ & $1.24451 \mathrm{E}+19$ \\
\hline 2005 & Oct. & $7.30334 \mathrm{E}+21$ & $1.45584 \mathrm{E}+21$ \\
\hline 2005 & Nov. & $2.63897 \mathrm{E}+19$ & $3.61799 \mathrm{E}+20$ \\
\hline 2005 & Dec. & $7.227 \mathrm{E}+20$ & $2.78612 \mathrm{E}+20$ \\
\hline 2006 & Jan. & $9.03744 \mathrm{E}+21$ & $2.38166 \mathrm{E}+19$ \\
\hline 2006 & Feb. & $2.84242 \mathrm{E}+20$ & $5.26544 \mathrm{E}+19$ \\
\hline 2006 & Mar. & $1.74146 \mathrm{E}+21$ & $7.01504 \mathrm{E}+19$ \\
\hline 2006 & Apr. & 4.97334E+19 & $6.19877 \mathrm{E}+20$ \\
\hline 2006 & May & $6.12295 \mathrm{E}+21$ & $4.58971 \mathrm{E}+21$ \\
\hline 2006 & Jun. & $6.12458 \mathrm{E}+21$ & $4.17313 \mathrm{E}+20$ \\
\hline 2006 & Jul. & $2.22845 \mathrm{E}+20$ & $1.10445 \mathrm{E}+19$ \\
\hline 2006 & Aug. & $2.56611 \mathrm{E}+20$ & $1.62649 \mathrm{E}+21$ \\
\hline 2006 & Sep. & $8.24293 \mathrm{E}+19$ & $3.0517 \mathrm{E}+20$ \\
\hline 2006 & Oct. & $1.55791 \mathrm{E}+20$ & $5.70032 \mathrm{E}+20$ \\
\hline 2006 & Nov. & $7.19683 \mathrm{E}+20$ & $3.23731 \mathrm{E}+19$ \\
\hline 2006 & Dec. & $1.84017 \mathrm{E}+22$ & $3.42244 \mathrm{E}+21$ \\
\hline
\end{tabular}


Table V. (continued).

\begin{tabular}{lccc}
\hline year & month & TM $\boldsymbol{0}_{\mathbf{0}}$ Etna $(\mathbf{d y n} \mathbf{c m})$ & TM $_{\mathbf{0}}$ Aeolian $($ dyn $\mathbf{~ c m})$ \\
\hline 2007 & Jan. & $1.75792 \mathrm{E}+19$ & $1.39637 \mathrm{E}+20$ \\
2007 & Feb. & $1.66358 \mathrm{E}+20$ & 0 \\
2007 & Mar. & $6.99842 \mathrm{E}+19$ & $3.04503 \mathrm{E}+20$ \\
2007 & Apr. & $3.07995 \mathrm{E}+20$ & $2.30905 \mathrm{E}+20$ \\
2007 & May & $4.97951 \mathrm{E}+20$ & $3.9762 \mathrm{E}+21$ \\
2007 & Jun. & $2.11475 \mathrm{E}+20$ & $1.2484 \mathrm{E}+20$ \\
2007 & Jul. & $4.4157 \mathrm{E}+18$ & $9.56001 \mathrm{E}+20$ \\
2007 & Aug. & $4.98609 \mathrm{E}+20$ & $4.57858 \mathrm{E}+20$ \\
2007 & Sep. & $1.75792 \mathrm{E}+19$ & $3.45058 \mathrm{E}+21$ \\
2007 & Oct. & $2.96098 \mathrm{E}+20$ & $2.60874 \mathrm{E}+20$ \\
2007 & Nov. & $5.99065 \mathrm{E}+19$ & $1.64468 \mathrm{E}+20$ \\
2007 & Dec. & $7.95694 \mathrm{E}+19$ & $1.39637 \mathrm{E}+20$ \\
\hline
\end{tabular}

\section{REFERENCES}

Acocella, V., B. Behncke, M. Neri and S. D'Amico (2003): Link between major flank slip and 2002-2003 eruption at Mt. Etna (Italy), Geophy. Res. Lett., 30, 2286, doi: 10.1029/2003GL018642.

Alparone, S., B. Behncke, S. Giammanco, M. Neri and E. Privitera (2005): Paroxysmal summit activity at Mt. Etna (Italy) monitored through continuous soil radon measurements, Geophys. Res. Lett., 32, L16307, doi: 10.1029/2005GL023352.

AzZARo, R., M.S. BARbano, R. Rigano and S. VinciguerRA (2001): Time seismicity patterns affecting local and regional fault systems in the Etna region: preliminary results for the period 1874-1913, J. Geol. Soc., Lond., 158, 561-572.

BARATTA, M. (1901): I terremoti d'Italia (Arnoldo Forni Editore, Bologna)

Barberi, G., O. Cocina, G. Neri, E. Privitera and S. SPAMPINATO (2000): Volcanological inferences from seismic-strain tensor computations at Mt. Etna Volcano, Sicily, Bull. Volcanol., 62, 318-330.

BARRIENTOS, S.E. (1994): Large thrust earthquakes and volcanic eruptions, Pure Appl. Geophys., 142, 225-237.

Basili, R., G. Valensise, P. Vannoli, P. Burrato, U. FraCASSI, S. Mariano, M.M. Tiberti and E. Boschi (2008): The Database of Individual Seismogenic Sources (DISS), version 3: summarizing 20 years of research on Italy's earthquake geology, Tectonophysics, 453, 20-43.

Bautista, B.C., M.L.P. Bautista, R.S. Stein, E.S. Barcelona, R.S. Punongbayan, E.P. Laguerta, A.R. RAsDAs, G. Ambubuyog and E.Q. Amin (1996): Relationship of regional and local structures to Mount Pinatubo activity, in Fire and Mud: Eruptions and La- hars of Mount Pinatubo, Philippines, edited by Newhall, C.G. and R.S. PunOngbayan (University of Washington Press, Washington, D.C.), pp. 351-369.

Bebbington, M.S. and C.D. LAI (1996): Statistical analysis of New Zealand volcanic occurrence data, J. Volcanol. Geoth. Res., 74, 101-110.

BeHNCKE, B. (2001): Volcanism in the Southern Apennines and Sicily, in Anatomy of an Orogen: the Apennines and Adjacent Mediterranean Basins, edited by VAI, G.B. and I.P. MARTINI (Kluwer Academic Publishers, Dordrecht), pp. 105-120.

Billi, A., G. Barberi, C. Faccenna, G. Neri, F. Pepe and A. Sulli (2006): Tectonics and seismicity of the Tindari Fault System, southern Italy: crustal deformations at the transition between ongoing contractional and extensional domains located above the edge of a subducting slab, Tectonics, 25, TC2006, doi: 10.1029/2004TC001763.

Billi A., D. Presti, C. Faccenna, G. Neri and B. OrecCHIO (2007): Seismotectonics of the Nubia plate compressive margin in the south Tyrrhenian region, Italy: clues for subduction inception, J. Geophys. Res., 112, B08302, doi: 10.1029/2006JB004837.

Boschi, E., E. Guidoboni, G. Ferrari, G. Valensise and P. GASPERINI (1997): Catalogo dei forti terremoti in Italia dal 461 a.C. al 1990, (Istituto Nazionale di Geofisica, Roma e Storia Geofisica e Ambiente, Bologna).

Branca, S., M. Coltelli, E. De Beni and J. Wijbrans (2008): Geological evolution of Mount Etna volcano (Italy) from earliest products until the first central volcanism (between 500 and $100 \mathrm{ka}$ ago) inferred from geochronological and stratigraphic data, Int. J. Earth Sci., 97, 135-152.

Brodsky, E., B. Sturtevant and H. Kanamori (1998): Earthquakes, volcanoes, and rectified diffusion, J. Geophys. Res., 103, 23827-23838. 
Brodsky, E., V. Karakostas and H. Kanamori (2000): A new observation of dynamically triggered regional seismicity: earthquakes in Greece following the August, 1999 Izmit, Turkey earthquake, Geophys. Res. Lett., 27, 2741-2744.

Caliro, S., A. Caracausi, G. Chiodini, M. Ditta, F. Italiano, M. Longo, C. Minopoli, P.M. NucCio, A. PAOnITA and A. Rizzo (2004): Evidence of a recent input of magmatic gases into the quiescent volcanic edifice of Panarea, Aeolian Islands, Italy, Geophys. Res. Lett., 31, L07619, doi: 10.1029/2003GL019359.

Calvari, S., L. Spampinato, L. Lodato, A.J.L. Harris, M.R. Patrick, J. Dehn and M.R. Burton (2005): Chronology and complex volcanic processes during the 2002-2003 flank eruption at Stromboli volcano (Italy) reconstructed from direct observations and surveys with a handheld thermal camera, J. Geophys. Res., 110, B02201, doi: 10.1029/2004JB003129.

Cardaci, C., S. Falsaperla, P. Gasperini, G. Lombardo, W. Marzocchi and F. Mulargia (1993): Crosscorrelation analysis of seismic and volcanic data at Mt Etna volcano, Italy, Bull. Volcanol., 55, 596-603.

CARR, M.J. (1977): Volcanic activity and great earthquakes at convergent plate margins, Science, 197, 655-657.

Cigolini, C., M. Laiolo and D. Coppola (2007): Earthquake-volcano interactions detected from radon degassing at Stromboli (Italy), Earth Planet. Sci. Lett., 257, 511-525.

D'Agostino, N. and G. Selvaggi (2004): Crustal motion along the Eurasia-Nubia plate boundary in the Calabrian Arc and Sicily and active extension in the Messina Straits from GPS measurements, J. Geophys. Res., 109, B11402, doi: 10.1029/2004JB002998.

DE Dolomieu, D.G. (1783): Voyage aux Iles de Lipari fait en 1781, (Cuchet, Paris).

Dellino, P. and K. Kyriakopoulos (2003): Phreatomagmatic ash from the ongoing eruption of Etna reaching the Greek island of Cefalonia, J. Volcanol. Geoth. Res., 126, 341-345.

Dewey, J.F., M.L. Helman, E. Turco, D.H.W. Hutton and D. KNotT (1989): Kinematics of the western Mediterranean, in Alpine Tectonics, edited by COWARD, M.P., D. Dietrich and R.G. PARK, Geological Society Special Publication, 45, 265-283.

Esposito, A., G. Giordano and M. AnZIDEI (2006): The 2002-2003 submarine gas eruption at Panarea volcano (Aeolian Islands, Italy): volcanology of the seafloor and implications for the hazard scenario, Mar. Geol., 227, 119-134.

Faccenna, C., C. Piromallo, A. Crespo-Blanc and L. JoLIVET (2004): Lateral slab deformation and the origin of the western Mediterranean arcs, Tectonics, 23, TC1012, doi: 10.1029/2002TC001488.

Faccenna, C., L. Civetta, M. D’Antonio, F. Funiciello, L. Margheriti and C. Piromallo (2005): Constraints on mantle circulation around the deforming Calabrian slab, Geophys. Res. Lett., 32, L06311, doi: 10.1029/2004GL021874.

Feuillet, N., M. Cocco, C. Musumeci and C. Nostro (2006): Stress interaction between seismic and volcanic activity at Mt Etna, Geophys. J. Int., 164, 697718.

Frazzetta, G., P.Y. Gillot, L. La VolPe and M.F. Sheri-
DAN (1984): Volcanic hazards at Fossa of Vulcano: data from the last 6000 years, Bull. Volcanol., 47, 105124.

FouQuÉ, F.A. (1865): Sur l'eruption de l'Etna du 1 fevrier 1865, Comptes Rendus de l'Academie des Sciences, 61, 210-211.

Gabbianelli, G., C. Romagnoli, P.L. Rossi and N. CALANCHI (1993): Marine geology of the PanareaStromboli area (Aeolian Archipelago, Southern Tyrrhenian sea), Acta Vulcanologica, 3, 11-20.

GHISETTI, F. (1979): Relazioni tra strutture e fasi trascorrenti e distensive lungo i sistemi Messina-Fiumefreddo, Tindari-Letojanni e Alia-Malvagna (Sicilia nord-orientale): uno studio microtettonico, Geologica Romana, 18, 23-58.

Gillot, P.Y., G. KiefFer and R. Romano (1994): The evolution of Mount Etna in the light of potassium-argon dating, Acta Vulcanologica, 5, 81-87.

GlasBy, G.P. and J. KASAHARA (2001): Influence of tidal effects on the periodicity of earthquake activity in diverse geological settings with particular emphasis on submarine hydrothermal systems, Earth-Sci. Rev., 52, 261-297.

Goes, S., D. Giardini, S. Jenny, C. Hollenstein, H.G. Kahle and A. Geiger (2004), A recent tectonic reorganization in the south-central Mediterranean, Earth Planet. Sci. Lett., 226, 335-345.

Gudmundsson, A. (2006): How local stresses control magma-chamber ruptures, dyke injections, and eruptions in composite volcanoes, Earth-Sci. Rev., 79, 1-31.

Gudmundsson, A. and S.L. BRENNER (2003): Loading of a seismic zone to failure deforms nearby volcanoes: a new earthquake precursor, Terra Nova, 15, 187-193.

Gudmundsson, A. and R.E.B. ANDREW (2007): Mechanical interaction between active volcanoes in Iceland, Geophys. Res. Lett., 34, L10310, doi: 10.1029/2007GL029873.

GvirtZMAN, Z. and A. Nur (1999): The formation of Mount Etna as the consequence of slab rollback, $\mathrm{Na}$ ture, 401, 782-785.

GVIRTZMAN, Z. and A. NuR (2001): Residual topography, lithospheric structure and sunken slabs in the central Mediterranean, Earth Planet. Sci. Lett., 187, 117-130.

HANKS, T.C. and H. KANAMORI (1979): A moment-magnitude scale, J. Geophys. Res., 84, 2348-2350.

HARRIS, A.J.L. and M. RIPEPE (2007): Regional earthquake as a trigger for enhanced volcanic activity: evidence from MODIS thermal data, Geophys. Res. Lett., 34, L02304, doi: 10.1029/2006GL028251.

JACQUES, E., G.C.P. KING, P. TAPPONNIER, J.C. RUEGG and I. MANIGHETTI (1996): Seismic activity triggered by stress changes after the 1978 events in the Asal rift, Djibouti, Geophys. Res. Lett., 23, 2481-2484.

JAQUET, O. and R. CARNIEL (2006): Estimation of volcanic hazards using geostatistical models, in Statistics in Volcanology edited by: H. MADER, S. COLES, C. CONNOR and L. CONNOR, (IAVCEI Publications IAV001, Geological Society of London), pp. 89-104

KANAMORI, H. and J.W. Givens (1982): Analysis of long period seismic waves excited by the May 18, eruption of Mount St. Helens: a terrestrial monopole?, J. Geophys. Res., 87, 5422-5432.

KIMURA, J. (1996): Near-synchronicity and periodicity of back-Arc propagation of Quaternary explosive volcan- 
ism in the southern segment of Northeastern Honshu Arc, Japan: A study facilitated by tephrochronology, Quat. Int., 34-36, 99-105.

KLEIN, F.W. (1982): Patterns of historical eruptions at Hawaiian volcanoes, J. Volcanol. Geoth. Res., 12, 1-35.

Lanzafame, G., M. Neri, V. Acocella, A. Billi, R. FuniCIELLO and G. GIORDANO (2003): Structural features of the July-August 2001 Mount Etna eruption: evidence for a complex magma supply system, J. Geol. Soc., London, 160, 531-544.

LEMARCHAND, N. and J.-R. GRASSO (2007): Interactions between earthquakes and volcano activity, Geophys. Res. Lett., 34, L24303, doi: 10.1029/2007GL031438.

LINDE, A.T. and I.S. SACKS (1998): Triggering of volcanic eruptions, Nature, 395, 888-890.

Madonia, P., C. Federico, P. Cusano, S. Petrosino, A. AIUPPA and S. GURRIERI (2008): Crustal dynamics of Mount Vesuvius from 1998 to 2005: Effects on seismicity and fluid circulation, J. Geophys. Res., 113, B05206, doi: 10.1029/2007JB005210.

Malinverno, A. and W.B.F. RYAN (1986): Extension in the Tyrrhenian Sea and shortening in the Apennines as result of arc migration driven by sinking of the lithosphere, Tectonics, 5, 227-254.

MangA, M. (2007): Did an earthquake trigger the May 2006 eruption of the Lusi Mud Volcano?, Eos Transactions of the American Geophysical Union, 88, 201, doi: 10.1029/2007EO180009.

Manga, M. and E. BRodsky (2006): Seismic triggering of eruptions in the far field: volcanoes and geysers, Annu. Rev. Earth Planet. Sci., 34, 263-291.

Marzocchi, W., R. Scandone and F. Mulargia (1993): The tectonic setting of Mount Vesuvius and the correlation between its eruptions and the earthquakes of the southern Apennines, J. Volcanol. Geoth. Res., 58, 27-41.

MarzocChi, W., E. CASAROTTI and A. Piersanti (2002): Modeling the stress variations induced by great earthquakes on the largest volcanic eruptions of the $20^{\text {th }}$ century, J. Geophys. Res., 107, 2320, doi: 10.1029/2001JB001391.

Marzocchi, W., ZacCarelli, L. and E. Boschi (2004): Phenomenological evidence in favor of a remote seismic coupling for large volcanic eruptions, Geophys. Res. Lett., 31, L04601, doi: 10.1029/2003GL018709.

McNutT, S.R. (1999): Eruptions of Pavolf Volcano, Alas$\mathrm{ka}$, and their possible modulation by ocean load and tectonic stresses: re-evaluation of the hypothesis based on new data from 1984-1998, Pure Appl. Geophys., 155, 701-712.

McNutt, S.R. and R.J. Beavan (1987): Eruptions of Pavlof Volcano and their possible modulation by ocean load and tectonic stresses, J. Geophys. Res., 92, 1150911523.

Mellors, R., D. Kilb, A. Aliyev, A. Gasanov and G. YeTIRMISHLI (2007): Correlations between earthquakes and large mud volcano eruptions, J. Geophys. Res., 112, B04304, doi: 10.1029/2006JB004489.

MerCALli, G. (1879): Contribuzioni alla geologia delle Isole Lipari, Atti della Società Italiana di Scienze Naturali, 22, 369-380.

Mercalli, G. (1888): L'Isola Vulcano e lo Stromboli dal 1886 al 1888, Atti della Società Italiana di Scienze Naturali, 31, 1-15.

Miklius, A. and P. Cervelli (2003), Interaction between
Kilauea and Mauna Loa, Nature, 421, 229.

Montelli, R., G. Nolet, F.A. Dahlen, G. Masters, E.R. ENGDAHL and S.-H. Hung (2004): Finite-frequency tomography reveals a variety of plumes in the mantle, Science, 303, 338-343.

Montone, P., M.T. Mariucci, S. Pondrelli and A. Amato (2004): An improved stress map for Italy and surrounding regions (Central Mediterranean), J. Geophys. Res., 109, B10410. doi: 10.1029/2003JB002703.

Mulargia, F., S. Tinti and E. Boschi (1985): A statistical analysis of flank eruptions on Etna volcano, J. Volcanol. Geoth. Res., 23, 263-272

Mulargia, F., P. Gasperini and W. Marzocchi (1991): Pattern recognition applied to volcanic activity: identification of the precursory patterns to Etna recent flank eruptions and periods of rest., J. Volcanol. Geoth. Res., 45, 187-196.

Nercessian, A., A. HiRn and M. SAPIN (1991): A correlation between earthquakes and eruptive phases at Mt Etna: an example and past occurrences, Geophys. J. Int., 105, 131-138.

Neri, G., G. Barberi, B. Orecchio and A. Mostaccio (2003): Seismic strain and seismogenic stress regimes in the crust of the southern Tyrrhenian region, Earth Planet. Sci. Lett., 213, 97-112.

Neri, G., G. Barberi, G. Oliva and B. Orecchio (2005): Spatial variations of seismogenic stress orientations in Sicily, south Italy, Phys. Earth Planet. Int., 148, 175191

Neri, G., G. Oliva, B. Orecchio and D. Presti (2006): Possible seismic gap within a highly seismogenic belt crossing Calabria and Eastern Sicily, Italy, Bull. Seism. Soc. Am., 96, 1321-1331.

Newhall, C.F. and S. Self (1982): The volcanic explosivity index (VEI): an estimate of explosive Magnitude for historical eruptions, J. Geophys. Res., 87, 1231-1238.

Nostro, C., R.S. Stein, M. Cocco, M.E. Belardinelli and W. MARZOCCHI (1998): Two-way coupling between Vesuvius eruptions and southern Apennine earthquakes, Italy, by elastic stress transfer, J. Geophys. Res., 103, 24487, doi: 10.1029/98JB00902.

OHTAKE, M. and H. NAKAHARA (1999): Seasonality of great earthquake occurrence at the northeastern margin of the Philippine Sea plate, Pure Appl. Geophys., 155, 689-700

Patacca, E., R. Sartori and P. Scandone (1992): Tyrrhenian basin and Apenninic arcs: kinematic relations since late Tortonian times, Mem. Soc. Geol. It., 45, 425-451.

Patane, D., P. De Gori, C. Chiarabba and A. BonaccorSo (2003): Magma ascent and the pressurization of Mount Etna's volcanic system, Science, 299, 20612063.

Pondrelli, S., C. Piromallo and E. Serpelloni (2004): Convergence vs. retreat in Southern Tyrrhenian Sea: Insights from kinematics, Geophys. Res. Lett., 31, L06611, doi: 10.1029/2003GL019223.

Ripepe, M., E. Marchetti, G. Ulivieri, A. Harris, J. Dehn, M. Burton, T. Caltabiano and G. Salerno (2005): Effusive to explosive transition during the 2003 eruption of Stromboli volcano, Geology, 33, 341-344.

RitTmanN, A. (1931): Der Ausbruch des Stromboli am 11 September 1930, Zeitschrift für Vulkanologie, 14, 47-77. RitTmanN, A. (1964): Vulkanismus und Tektonik des Ätna, 
Geologische Rundschau, 53, 788-800.

Sharp, A.D.L., G. LOMBARdo and P.M. DAVIS (1981): Correlation between eruptions of Mount Etna, Sicily, and regional earthquakes as seen in historical records from AD 1582, Geophys. J. Int., 65, 507-523.

SieberT, L. and T. Simkin (2002): Volcanoes of the World: an Illustrated Catalog of Holocene Volcanoes and their Eruptions. Smithsonian Institution, Global Volcanism Program Digital Information Series, GVP-3, (http://www.volcano.si.edu/world/).

SILVESTRI, O. (1867): I fenomeni vulcanici presentati dall'Etna nel 1863-64-65-66, Atti dell'Accademia Gioenia di Scienze Naturali in Catania, 23, 55-317.

Silvestri, O. (1890): Etna, Sicilia ed isole vulcaniche adiacenti sotto il punto di vista dei fenomeni eruttivi e geodinamici avvenuti durante l'anno 1889, Atti dell'Accademia Gioenia di Scienze Naturali in Catania, 66, 222-249.

Silvestri, O. (1893): L'eruzione dell'Etna del 1886, Atti dell'Accademia Gioenia di Scienze Naturali in Catania, 69, 1-37.

Simkin, T. and L. SiEberT (1994): Volcanoes of the World, (Geoscience Press, Tucson).

Tanguy, J.-C., M. Condomines, M. Le Goff, V. Chillemi, S. LA Delfa and G. PATANE (2007): Mount Etna eruptions of the last 2,750 years: revised chronology and location through archeomagnetic and ${ }^{226} \mathrm{Ra}^{230} \mathrm{Th}$ dating, Bull. Volcanol., 70, 55-83.

Tortorici, L., G. Ventura, R. Mazzuoli and C. Monaco (1995): Strutture del settore orientale dell'Arcipelago Eoliano: interpretazione tettonica e modellizzazione numerica, Studi Geologici Camerti, 1995(2), 455-466.
TRYgGVASSON, E. (1973): Seismicity, earthquake swarms, and plate boundaries in the Iceland region, Bull. Seism. Soc. Am., 63, 1327-1348.

Ventura, G., G. Vilardo, G. Milano and N.A. Pino (1999): Relationships among crustal structure, volcanism and strike-slip tectonics in the Lipari-Vulcano Volcanic Complex (Aeolian Islands, southern Tyrrhenian Sea, Italy), Phys. Earth Planet. Int., 116, 31-52.

WALTER, T.R. and F. AMELUNG (2004): Influence of volcanic activity at Mauna Loa, Hawaii, on earthquake occurrence in the Kaoiki Seismic Zone, Geophys. Res. Lett., 31, L07622, doi:10.1029/2003GL019131.

WALTER, T.R. and F. Amelung (2006): Volcano-earthquake interaction at Mauna Loa volcano, Hawaii, J. Geophys. Res., 111, B05204, doi: 10.1029/2005JB003861.

WALter, T.R. and F. AmELung (2007), Volcanic eruptions following $M \geq 9$ megathrust earthquakes: implications for the Sumatra-Andaman volcanoes, Geology, 35, 539-542.

Walter, T.R., V. Acocella, M. Neri and F. Amelung (2005), Feedback processes between magmatic events and flank movement at Mount Etna (Italy) during the 2002-2003 eruption, J. Geophys. Res., 110, B10205, doi: 10.1029/2005JB003688.

WALter, T.R., R. WANG, M. ZIMMER, H. Grosser, B. LÜHR and A. RATDOMOPURBo (2007), Volcanic activity influenced by tectonic earthquakes: static and dynamic stress triggering at Mt. Merapi, Geophys. Res. Lett., 34, L05304, doi: 10.1029/2006GL028710.

(received February 11, 2008; accepted June 12, 2008) 